\title{
Synthesis of Tridentate $[1,2,4]$ Triazinyl-Pyridin-2-yl Indole Lewis Basic Complexants via Pd-catalyzed Suzuki-Miyaura Cross-Coupling
}

\section{Supporting Information}

\author{
Zachary Z. Gulledge, Mariah L. Tedder, Kyle R. Lyons, and Jesse D. Carrick
}

Department of Chemistry, Tennessee Technological University, 55 University Drive, Cookeville, TN 38505-0001

\section{For Table of Contents}

S1-S2

Figure S1. 1,2-bis-[4-(3-methyl-butyl)-phenyl]-ethane-1,2-dione ${ }^{1} \mathbf{H}$ NMR

Figure S2. 1,2-bis-[4-(3-methyl-butyl)-phenyl]-ethane-1,2-dione ${ }^{13} \mathbf{C}$ NMR

S3

S4

Figure S3. 3-(6-Bromo-pyridin-2-yl)-5,6-bis-[4-(3-methyl-butyl)-phenyl]-[1,2,4]triazine ${ }^{1} \mathrm{H}$ NMR

Figure S4. 3-(6-Bromo-pyridin-2-yl)-5,6-bis-[4-(3-methyl-butyl)-phenyl]-[1,2,4]triazine ${ }^{13} \mathrm{C}$ NMR

Figure S5. Compound $2{ }^{1} \mathrm{H}$ NMR

Figure S6. Compound $2{ }^{13} \mathrm{C}$ NMR

Figure S7. Compound $3^{1} \mathrm{H}$ NMR

Figure S8. Compound $3{ }^{13} \mathrm{C}$ NMR

Figure S9. Compound $4^{1} \mathrm{H}$ NMR

Figure S10. Compound $4{ }^{13} \mathrm{C}$ NMR

Figure S11. Compound $5{ }^{1} \mathrm{H}$ NMR

Figure S12. Compound $5{ }^{13} \mathrm{C}$ NMR

Figure S13. Compound $6^{1} \mathrm{H}$ NMR

Figure S14. Compound $6{ }^{13} \mathrm{C}$ NMR

Figure S15. Compound $7^{1} \mathrm{H}$ NMR

Figure S16. Compound $7{ }^{13} \mathrm{C}$ NMR

Figure S17. Compound $8^{1} \mathrm{H}$ NMR

Figure S18. Compound $8{ }^{13} \mathrm{C}$ NMR

Figure S19. Compound $9^{1} \mathrm{H}$ NMR

Figure S20. Compound $9{ }^{13} \mathrm{C}$ NMR

Figure S21. Compound $10^{1} \mathrm{H}$ NMR

Figure S22. Compound $10{ }^{13} \mathrm{C}$ NMR

Figure S23. Compound $11^{1} \mathrm{H}$ NMR

Figure S24. Compound $11{ }^{13} \mathrm{C}$ NMR

Figure S25. Compound $13^{1} \mathrm{H}$ NMR

Figure S26. Compound $13{ }^{13} \mathrm{C}$ NMR

Figure S27. Compound $14{ }^{1} \mathrm{H}$ NMR

Figure S28. Compound $14{ }^{13} \mathrm{C}$ NMR

Figure S29. Compound $15^{1} \mathrm{H}$ NMR

Figure S30. Compound $15{ }^{13} \mathrm{C}$ NMR

Figure S31. Compound $16{ }^{1} \mathrm{H}$ NMR

Figure S32. Compound $16{ }^{13} \mathrm{C}$ NMR

Figure S33. Compound $17^{1} \mathrm{H}$ NMR

\section{S5}

56

S7

S8

59

S10

S11

S12

$\mathrm{S} 13$

S14

S15

S16

S17

S18

S19

S20

S21

S22

$\mathrm{S} 23$

S24

S25

S26

S27

S28

S29

S30

S31

S32

S33

S34

S35 
For Table of Contents, cont.

Figure S34. Compound $17{ }^{13} \mathrm{C}$ NMR $\quad$ S36

$\begin{array}{ll}\text { Figure S35. Compound } 18{ }^{1} \mathrm{H} \text { NMR } & \text { S37 }\end{array}$

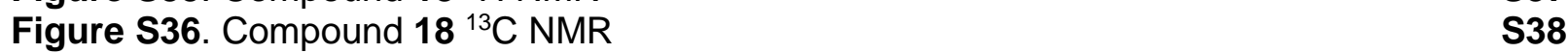

Figure S37. Compound $19^{1} \mathrm{H}$ NMR $\quad$ S39

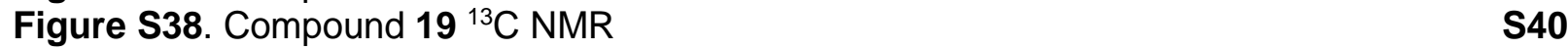

Figure S39. Compound $20^{1} \mathrm{H}$ NMR $\quad S 41$

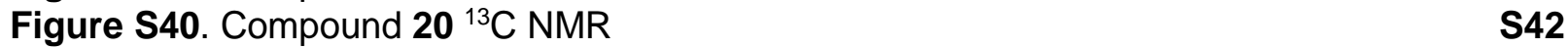

Figure S41. Compound $21^{1} \mathrm{H}$ NMR $\quad$ S43

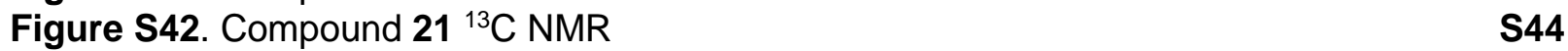

Figure S43. Compound $22{ }^{1} \mathrm{H}$ NMR $\quad S 45$

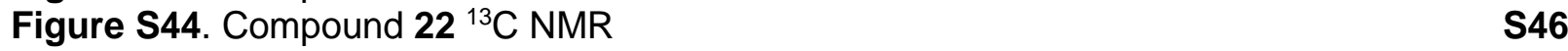

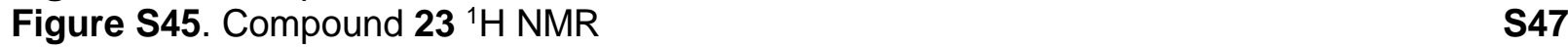

Figure S46. Compound $23{ }^{13} \mathrm{C}$ NMR $\quad S 48$

Figure S47. Compound $24^{1} \mathrm{H}$ NMR $\quad S 49$

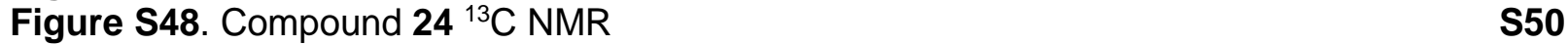

Figure S49. Compound $25^{1} \mathrm{H}$ NMR $\quad$ S51

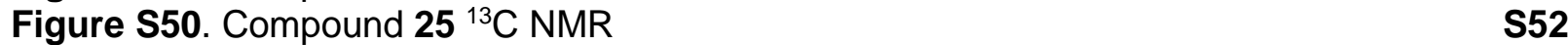

Figure S51. Compound $26^{1} \mathrm{H}$ NMR $\quad$ S53

Figure S52. Compound $26{ }^{13} \mathrm{C}$ NMR

Figure S53. Chromatogram of 1,2-bis-[4-(3-methyl-butyl)-phenyl]-ethane-1,2-dione $\quad$ S55

Figure S54. Chromatogram of 3-(6-Bromo-pyridin-2-yl)-5,6-bis-[4-(3-methyl-butyl)-phenyl]$[1,2,4]$ triazine

S56

Figure S55. Chromatogram of $2 \quad S 57$

$\begin{array}{ll}\text { Figure S56. Chromatogram of } 3 & \text { S58 }\end{array}$

Figure S57. Chromatogram of $4 \quad S 59$

Figure S58. Chromatogram of $5 \quad S 60$

Figure S59. Chromatogram of $6 \quad S 61$

Figure S60. Chromatogram of $7 \quad S 62$

Figure S61. Chromatogram of $8 \quad S 63$

Figure S62. Chromatogram of $9 \quad 564$

Figure S63. Chromatogram of $10 \quad 565$

Figure S64. Chromatogram of $11 \quad S 66$

$\begin{array}{ll}\text { Figure S65. Chromatogram of } 13 & \text { S67 }\end{array}$

Figure S66. Chromatogram of $14 \quad S 68$

Figure S67. Chromatogram of $15 \quad S 69$

$\begin{array}{ll}\text { Figure S68. Chromatogram of } 16 & \mathrm{S70}\end{array}$

Figure S69. Chromatogram of $17 \quad 571$

Figure S70. Chromatogram of $18 \quad S 72$

Figure S71. Chromatogram of $19 \quad S 73$

Figure S72. Chromatogram of $20 \quad 574$

Figure S73. Chromatogram of $21 \quad S 75$

Figure S74. Chromatogram of $22 \quad S 76$

$\begin{array}{ll}\text { Figure S75. Chromatogram of } 23 & \text { S77 }\end{array}$

Figure S76. Chromatogram of $24 \quad 578$

$\begin{array}{ll}\text { Figure S77. Chromatogram of } 25 & \text { S79 }\end{array}$

$\begin{array}{lr}\text { Figure S78. Chromatogram of } 26 & \mathbf{S 8 0}\end{array}$ 


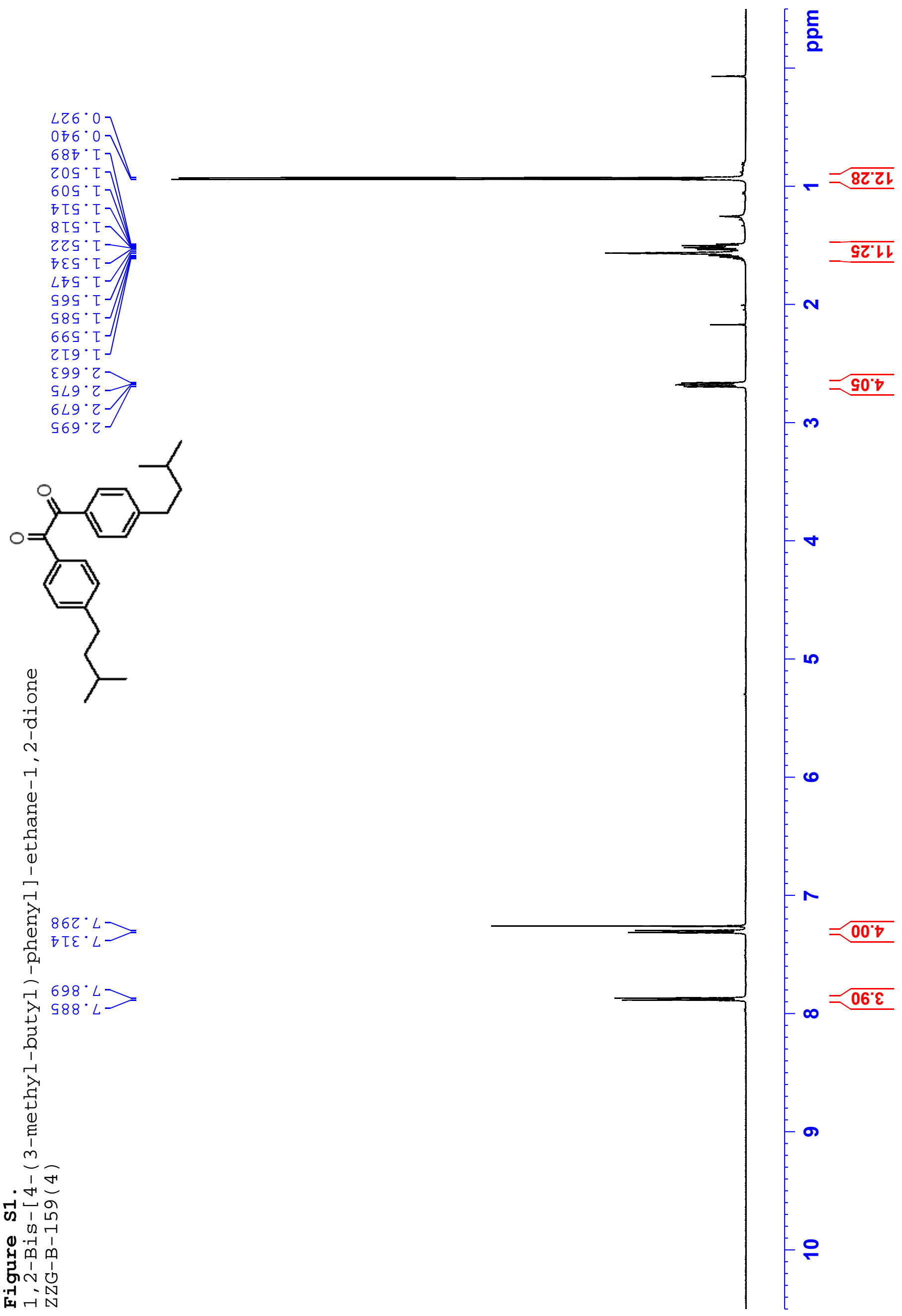




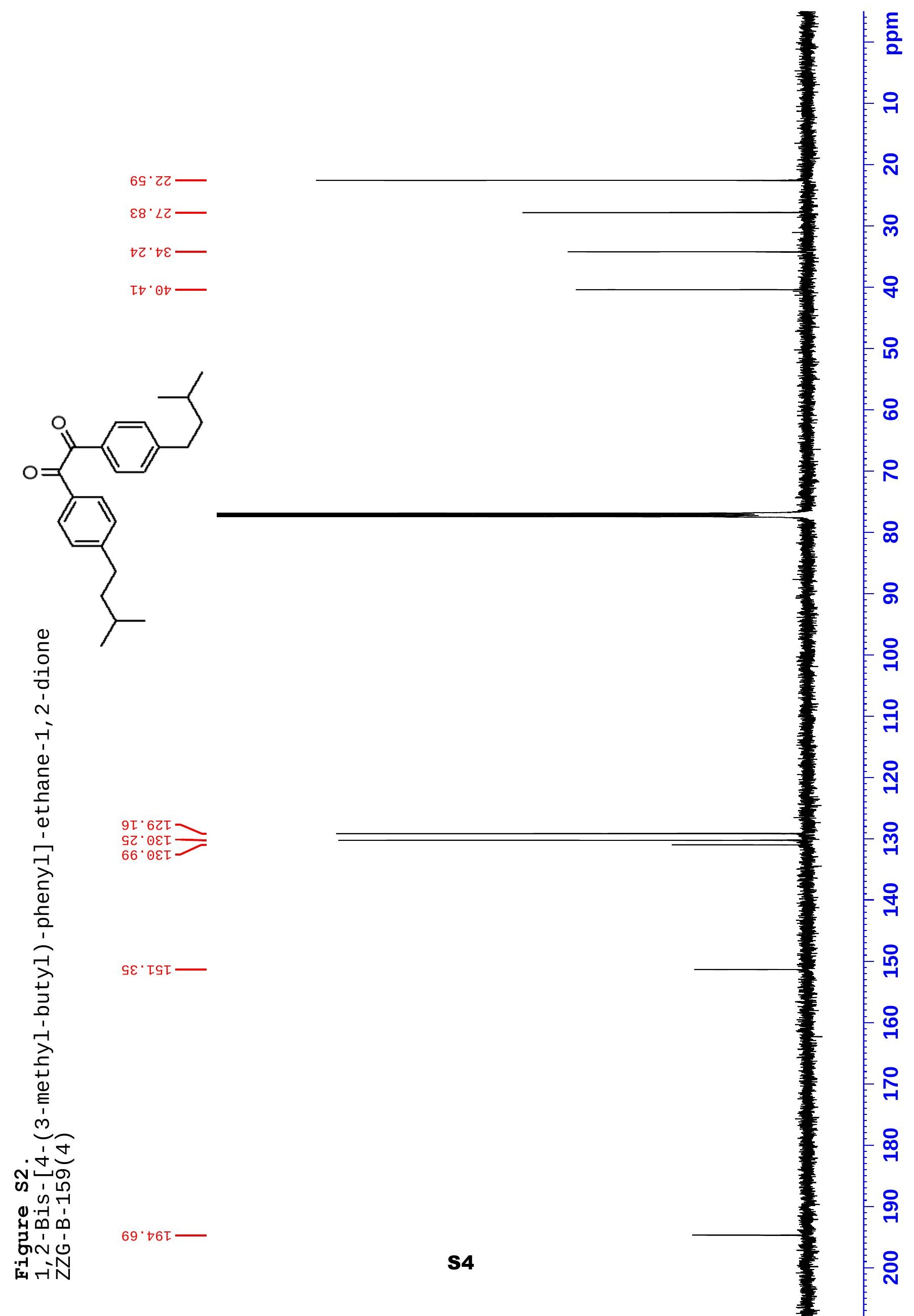




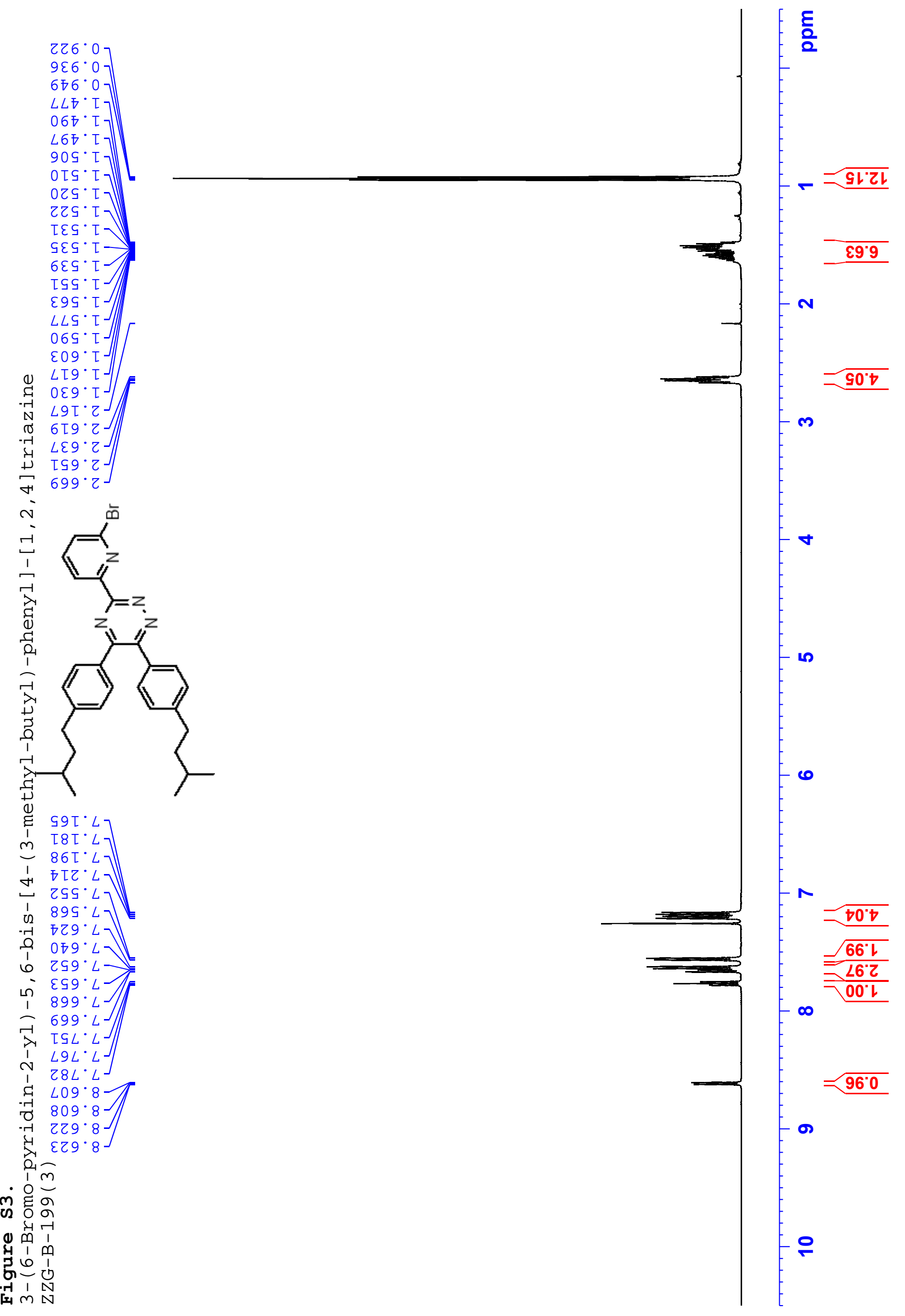




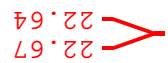

$6 L \angle Z \square$

$\angle 8 \cdot \angle Z \longrightarrow$

โ8. $\varepsilon \varepsilon \longrightarrow$

$8 t^{\circ} \cdot 0 t>$
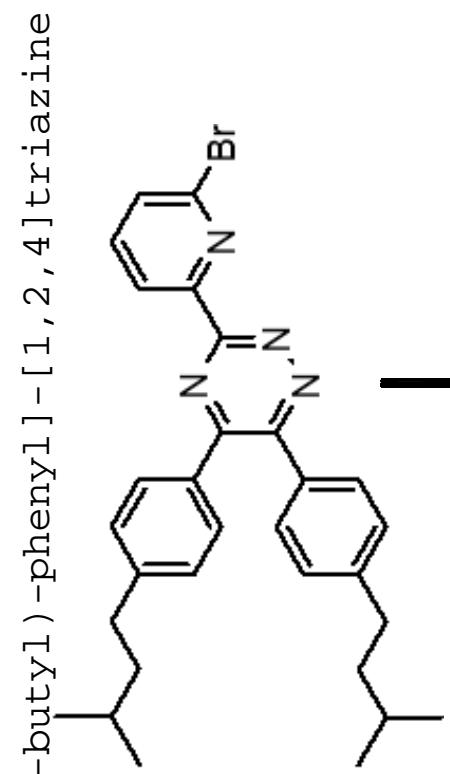

竞

'่ $\angle 6^{\circ} Z Z T-$

m) $\quad$ \& $8.8 Z T$

$\checkmark \quad \angle S^{\circ} 62 \mathrm{ZT}$

UL

is $\quad 6 \sigma^{\circ}$ ZEL

.-1 $82^{\circ} 6 \varepsilon \tau$

$\begin{array}{ll}0 & \tau \theta^{\circ} \varepsilon t I- \\ 0 & t t^{\circ} \operatorname{stI}=\end{array}$

i) $6 L \cdot 9 t 5$

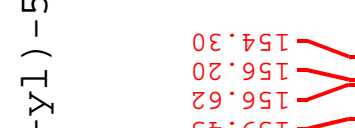

ì

I

$\stackrel{\cdot \frac{9}{.1}}{.}$

듬

는

ำ

$\therefore$ 음

ฟี ठิ ब

1

凶ा

4 '

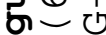

क्ष

오

요

우

웅

8

웃

요

8

욱

욱

ํำ

옥

욱

욱

욱

옥

$\stackrel{\circ}{\infty}$

옥

윰 


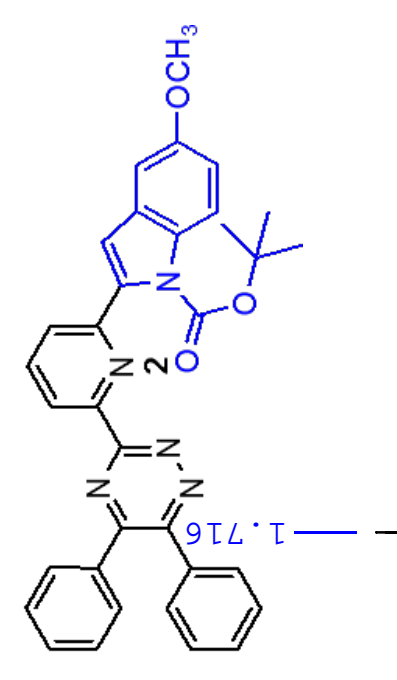

응

1'

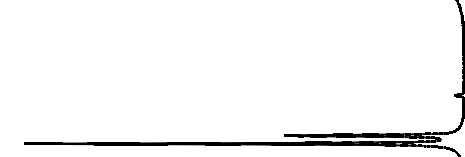

$\angle L E^{\prime} \angle$

ZSt' $L$

$\left.\odot \angle \nabla^{\circ} \angle\right]$

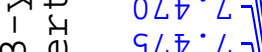

$L^{\prime}+0 \nabla 9^{\circ} L$

ᄃ

సै

त्त

ㄴ.

$\left.\angle 98^{\circ} L\right]$

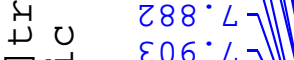

$\checkmark न 6^{\circ} \quad 8\left[6^{\circ} \mathrm{L}\right.$

N

더응 8t6. L

더는 $\varepsilon 96^{\circ} \mathrm{L}$

' ซ 60I'8

तु 0 गटL.8

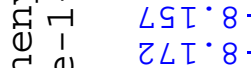

드워 $82 \varepsilon .8$

'등 응 $\varepsilon \nabla \varepsilon$ ' 8

. $匚 \mathcal{C} \angle t G^{\circ} 8$

ص

ऽ

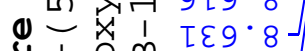

너요

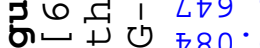

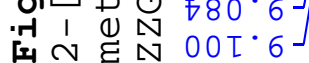




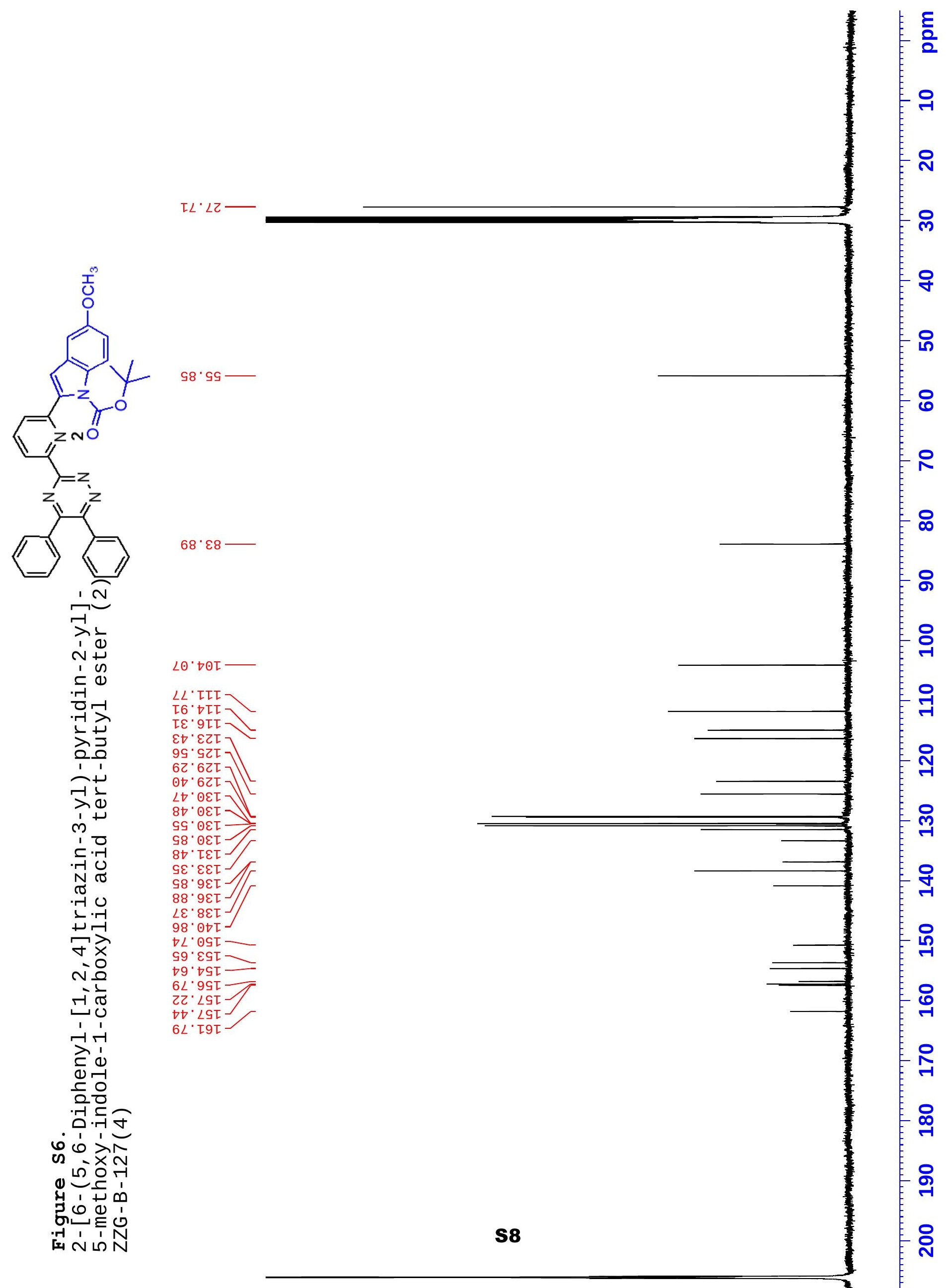




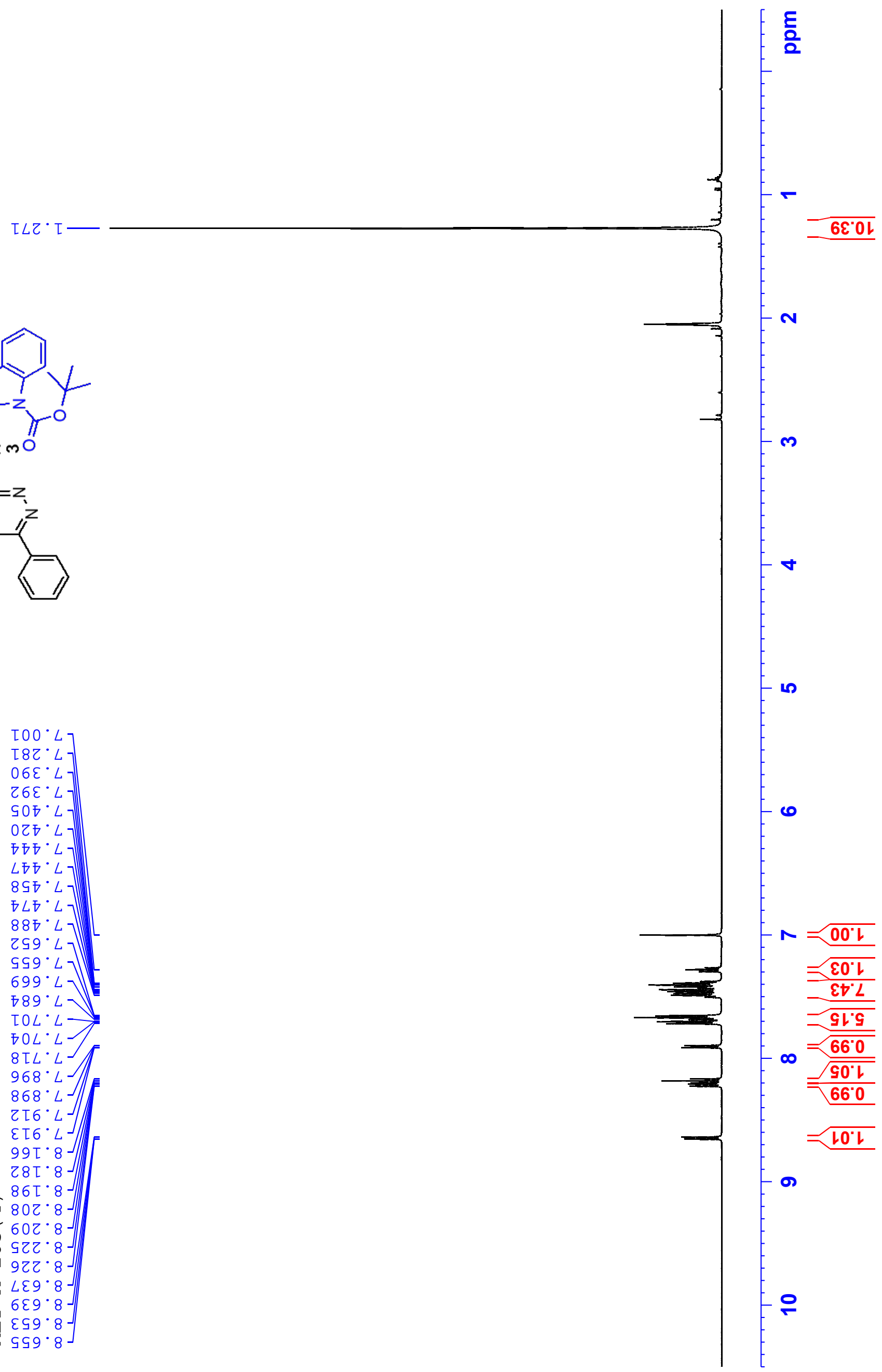




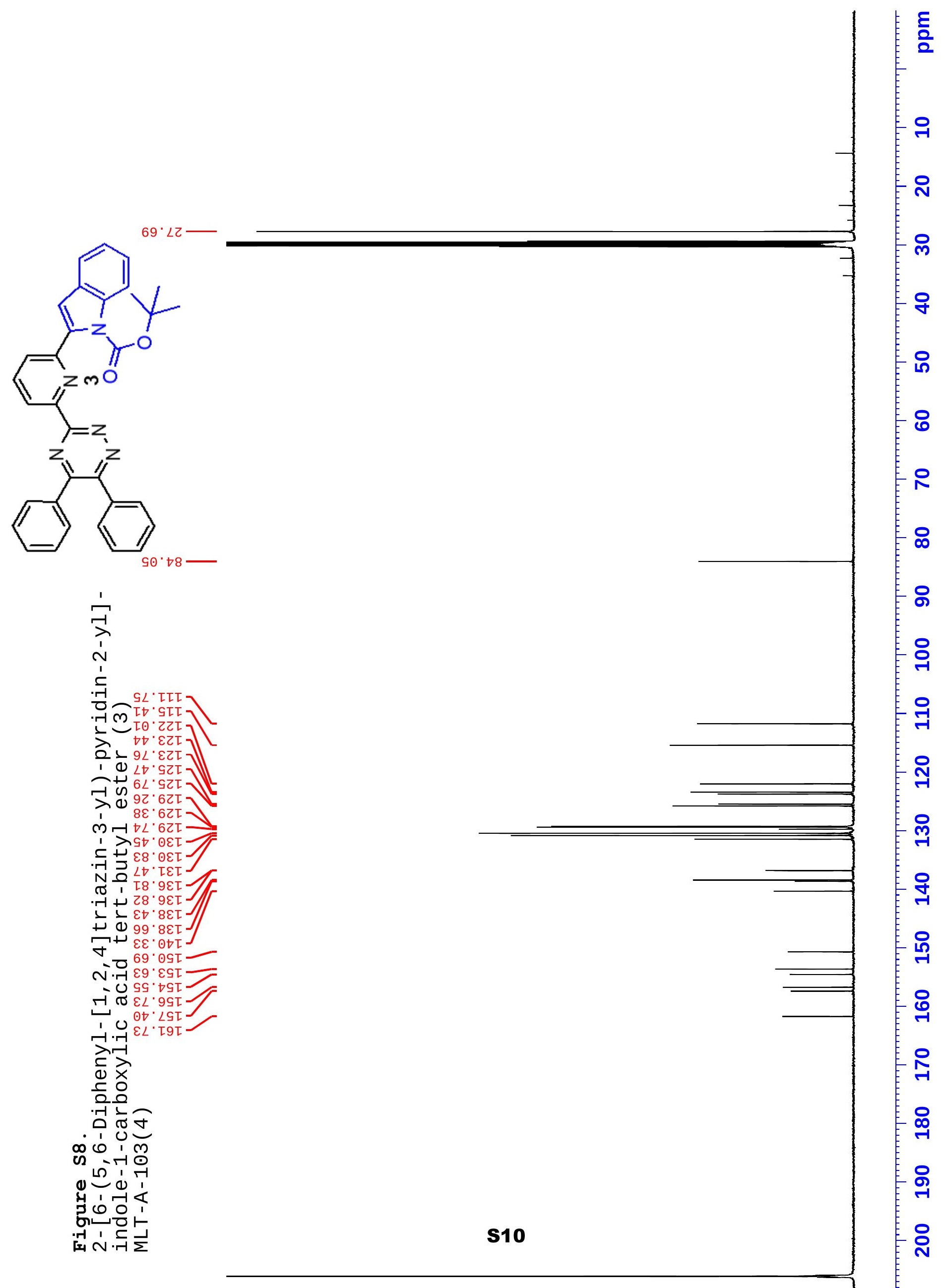




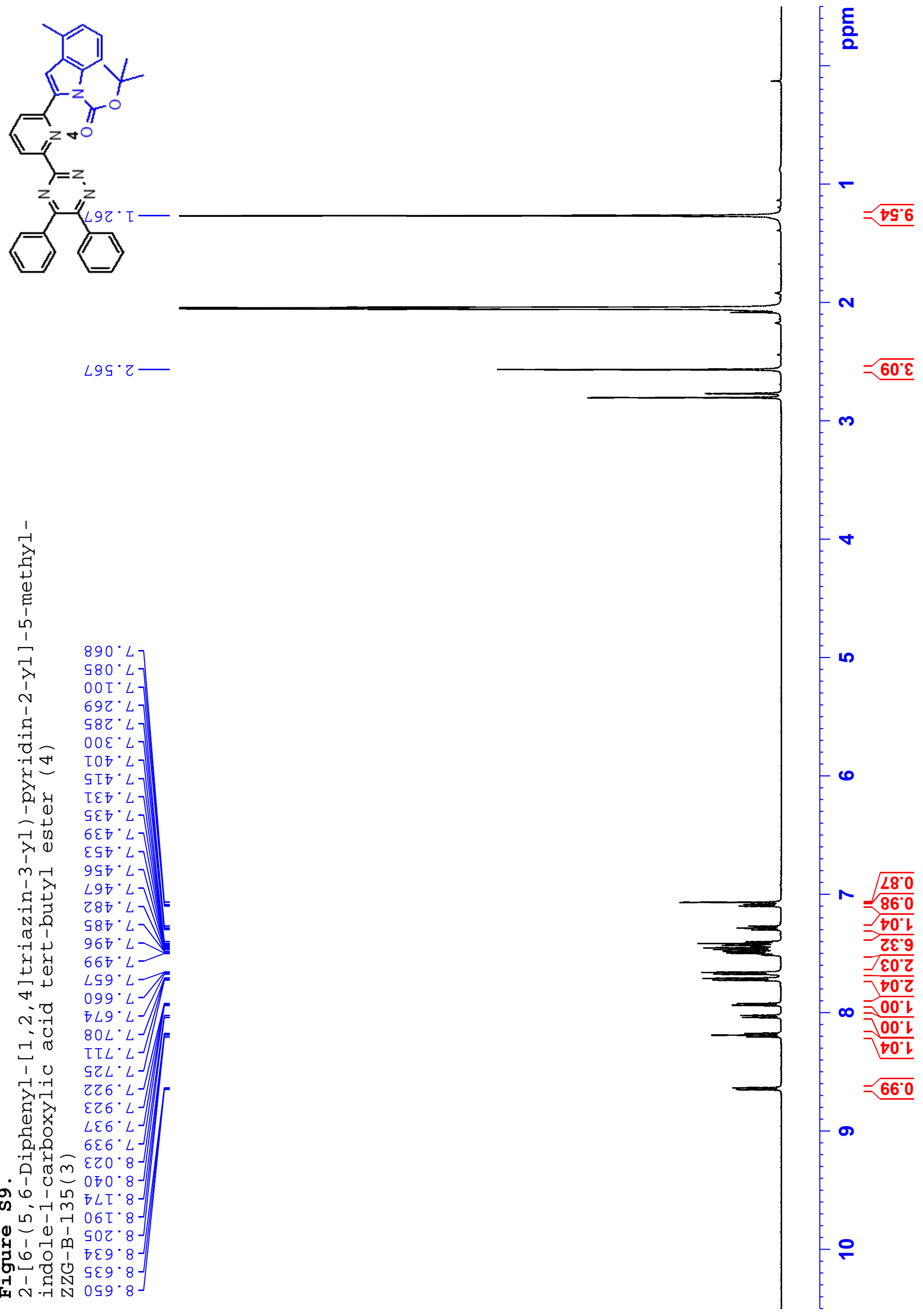




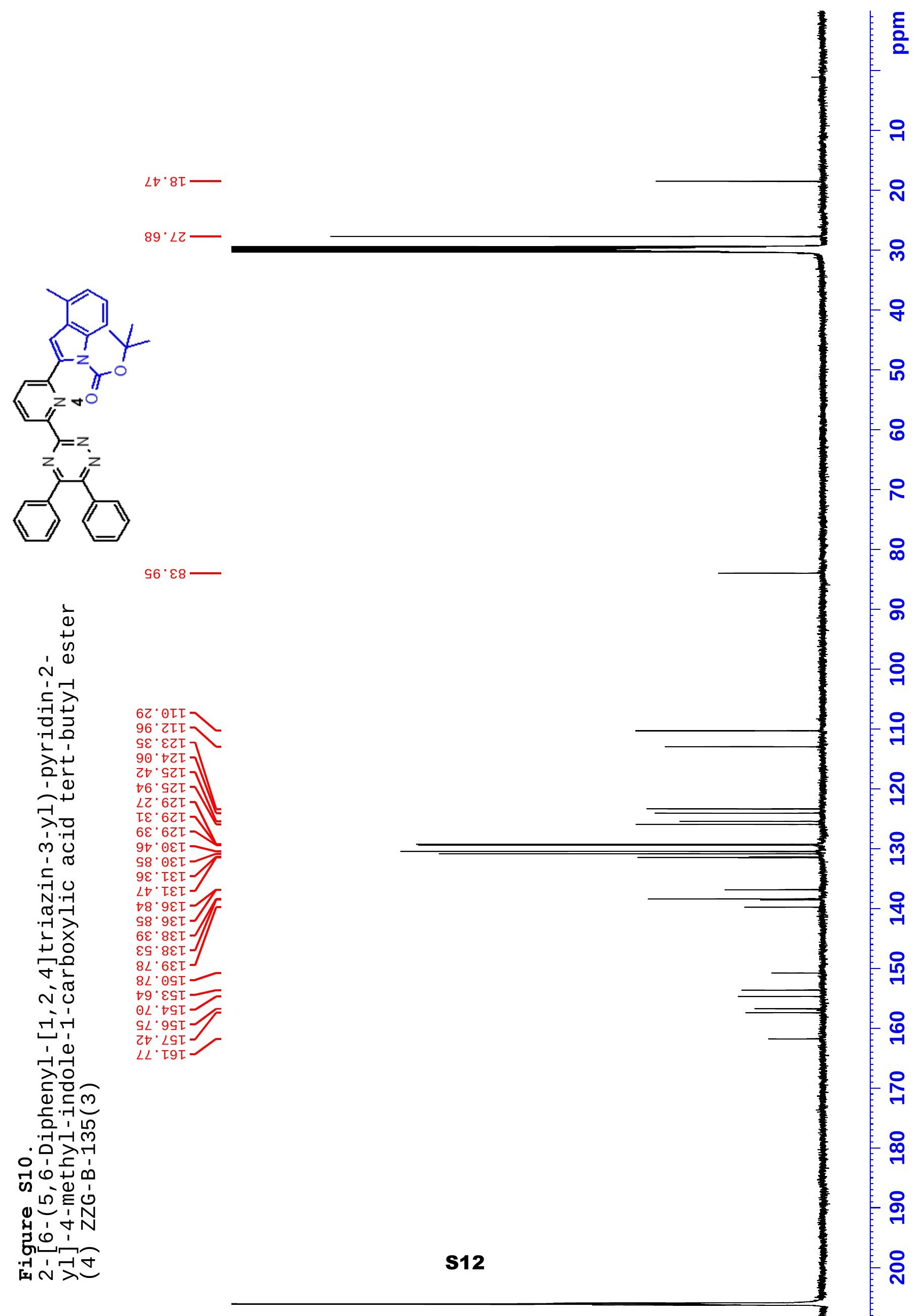




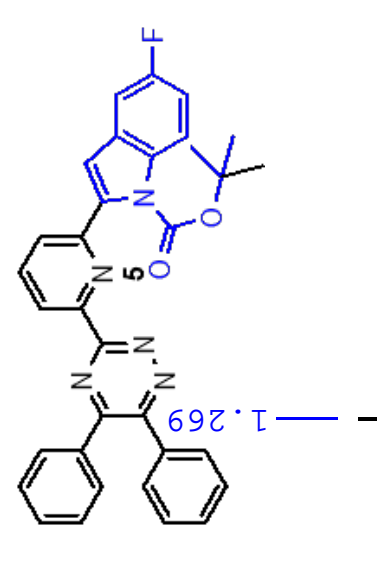

틍

$\varepsilon \varepsilon^{\prime 6}$
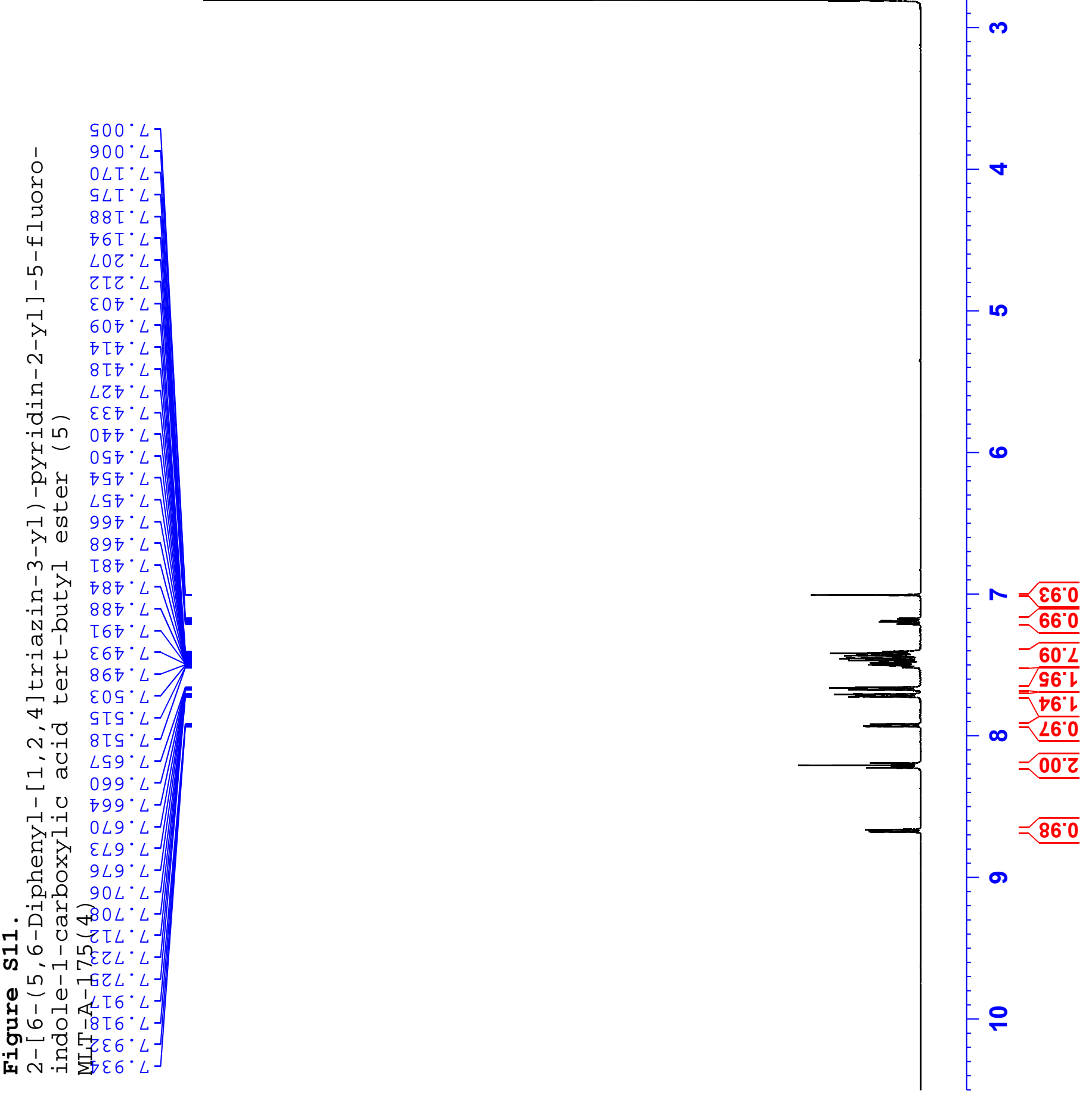


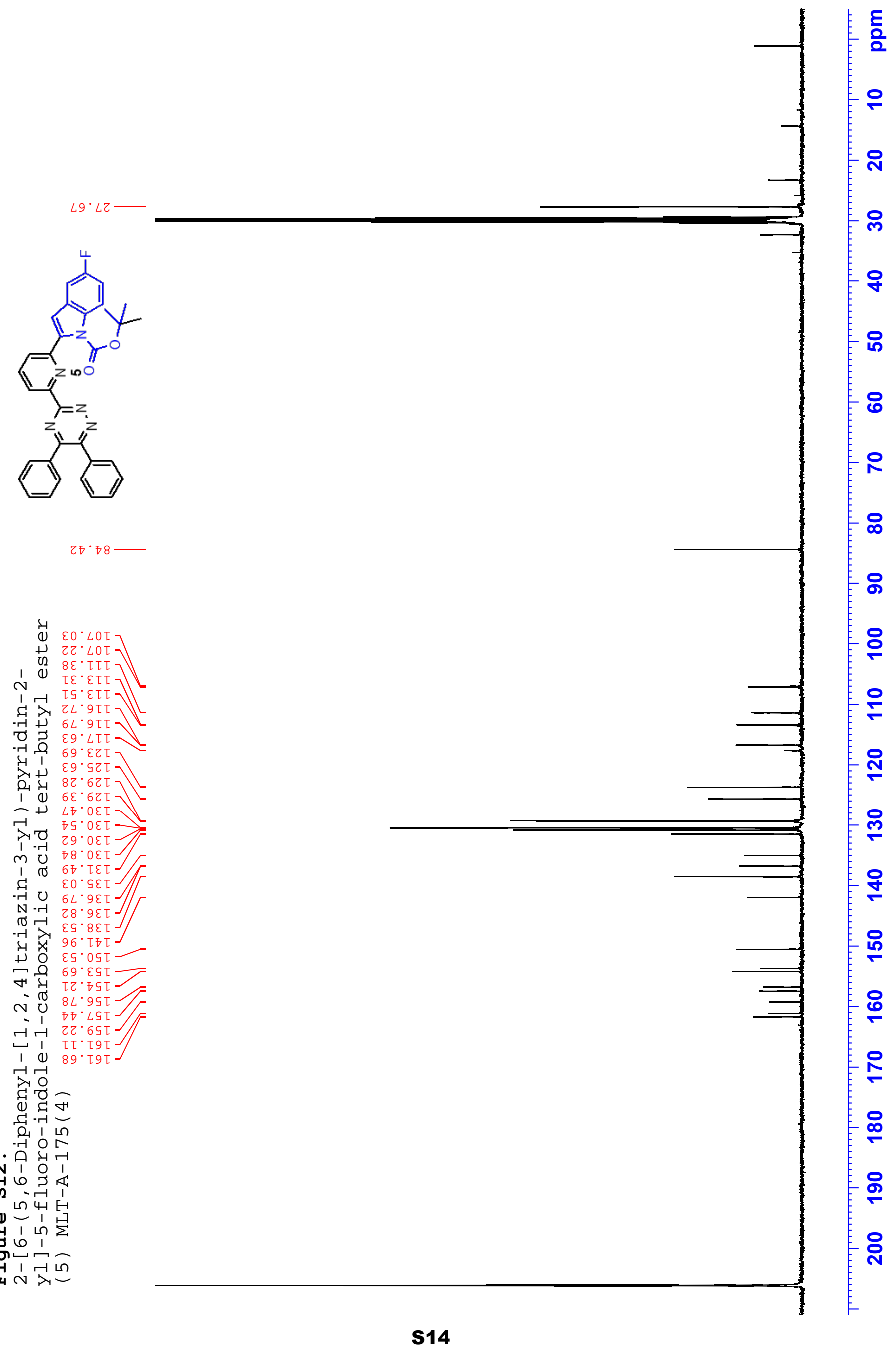



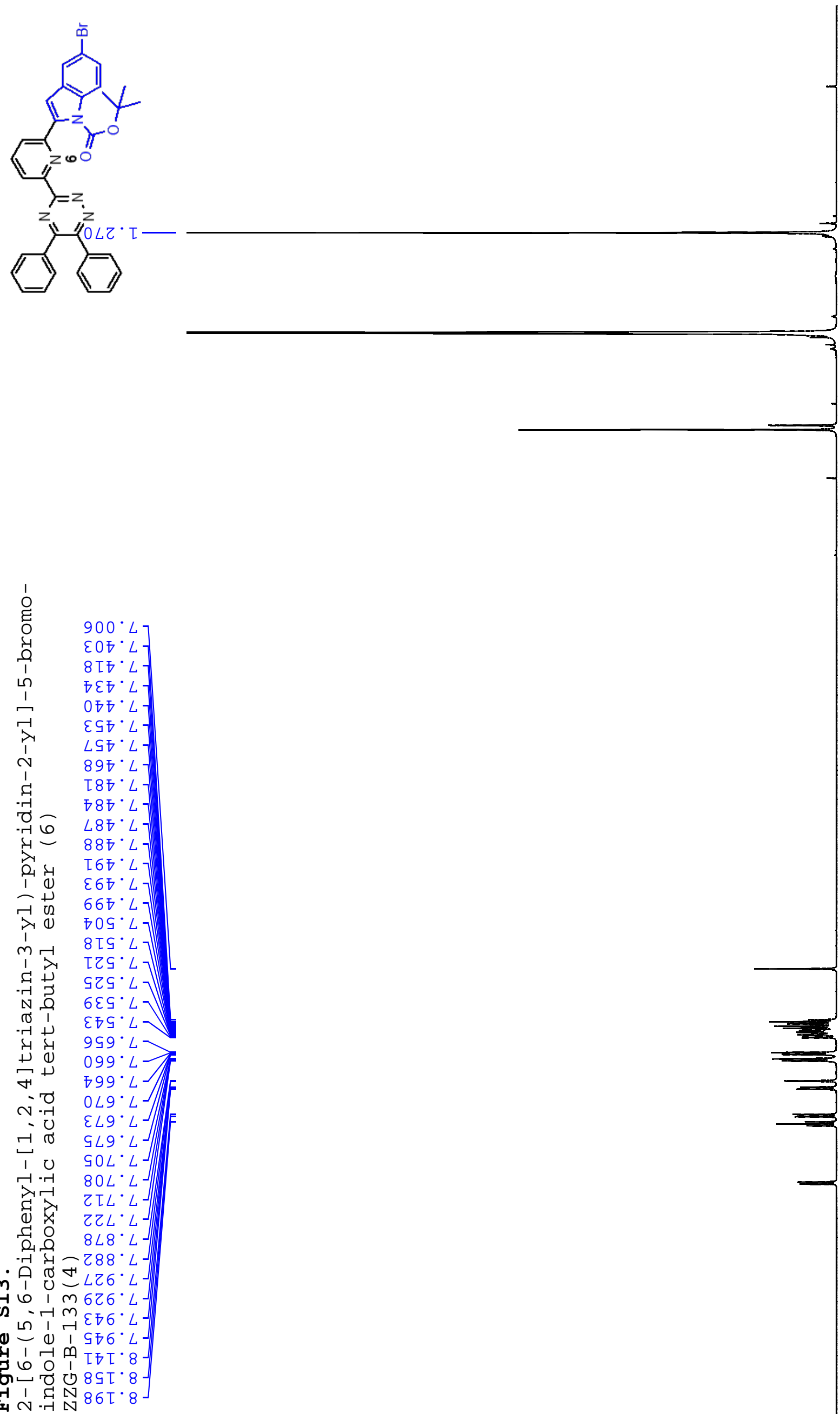


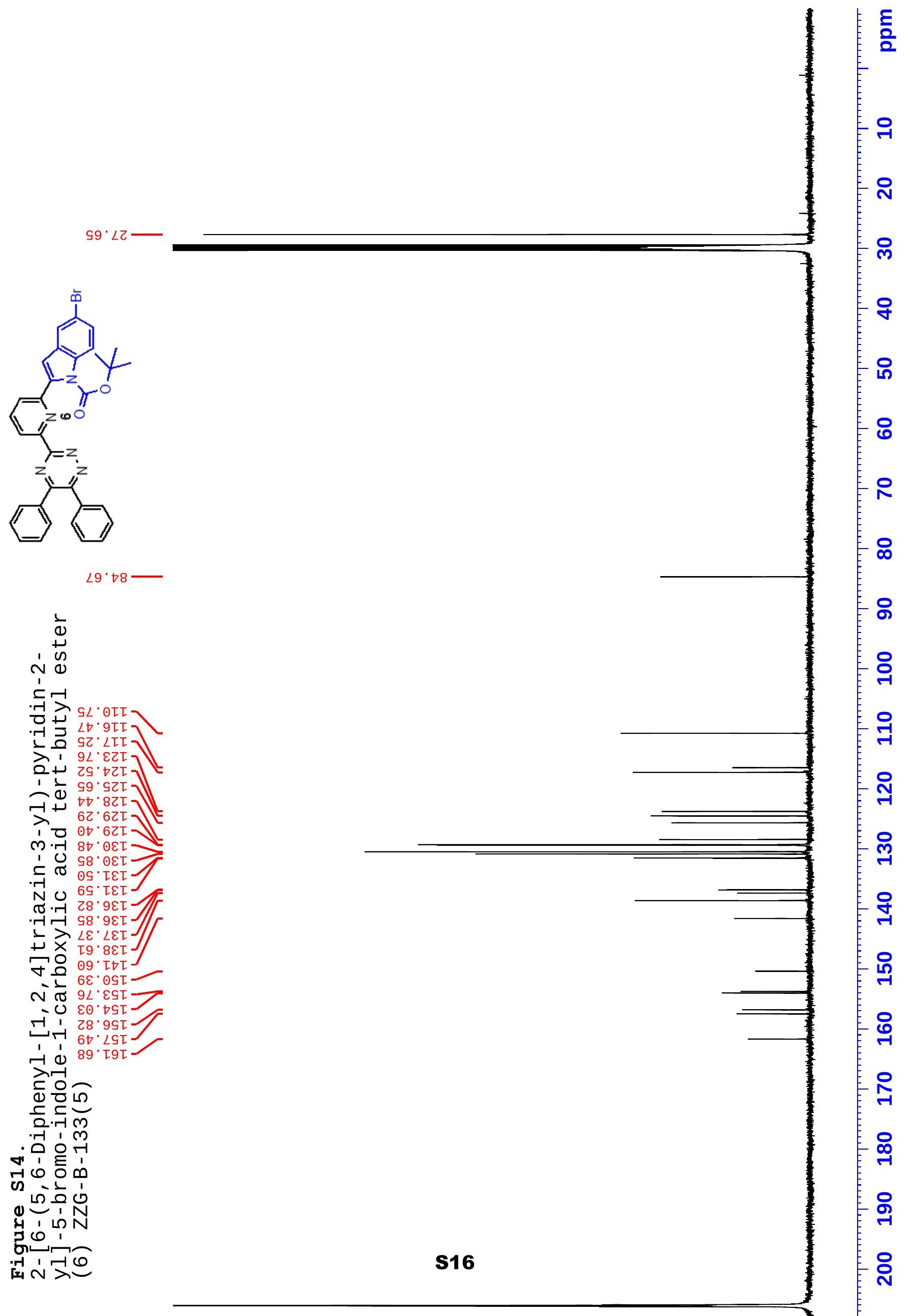




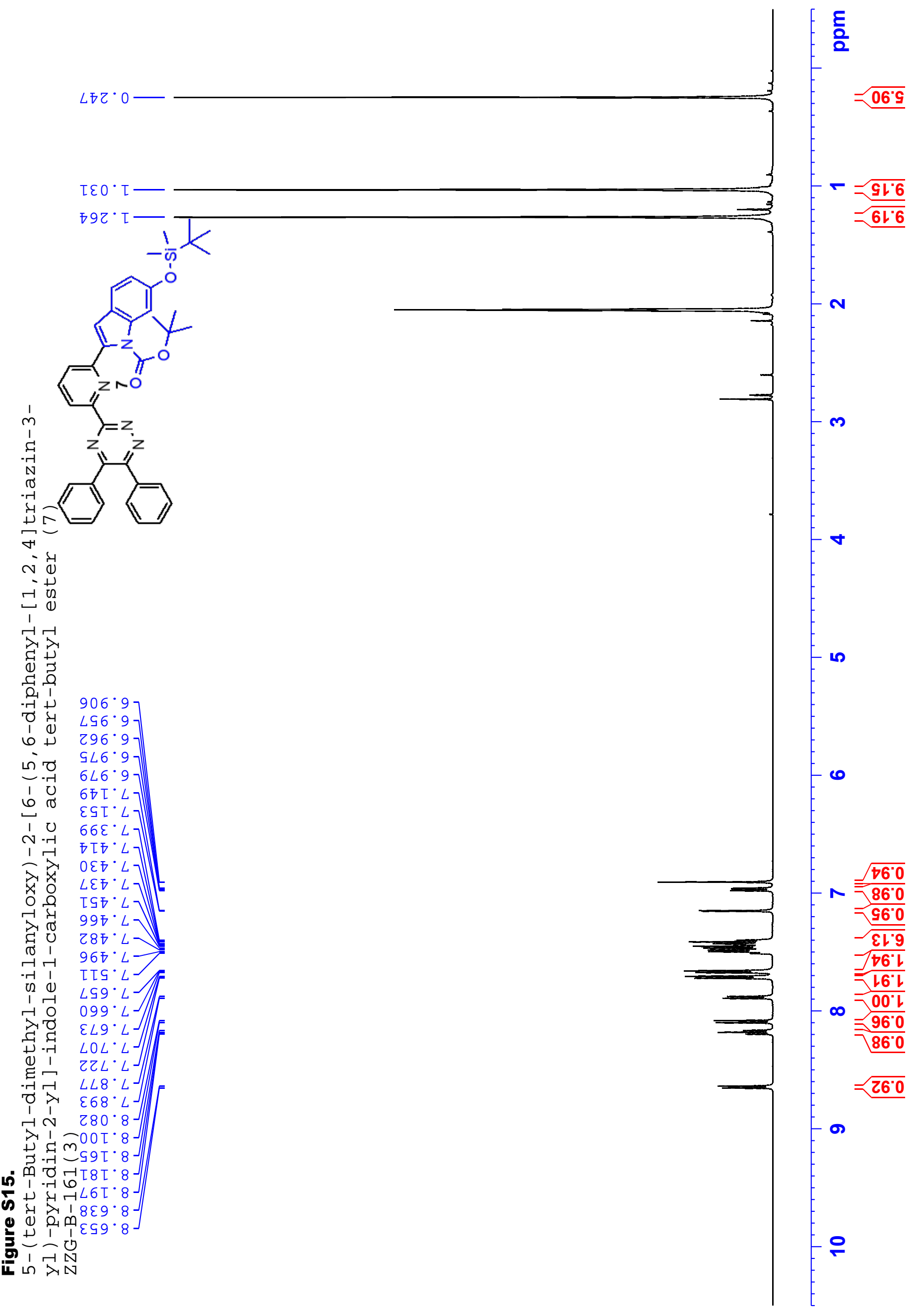



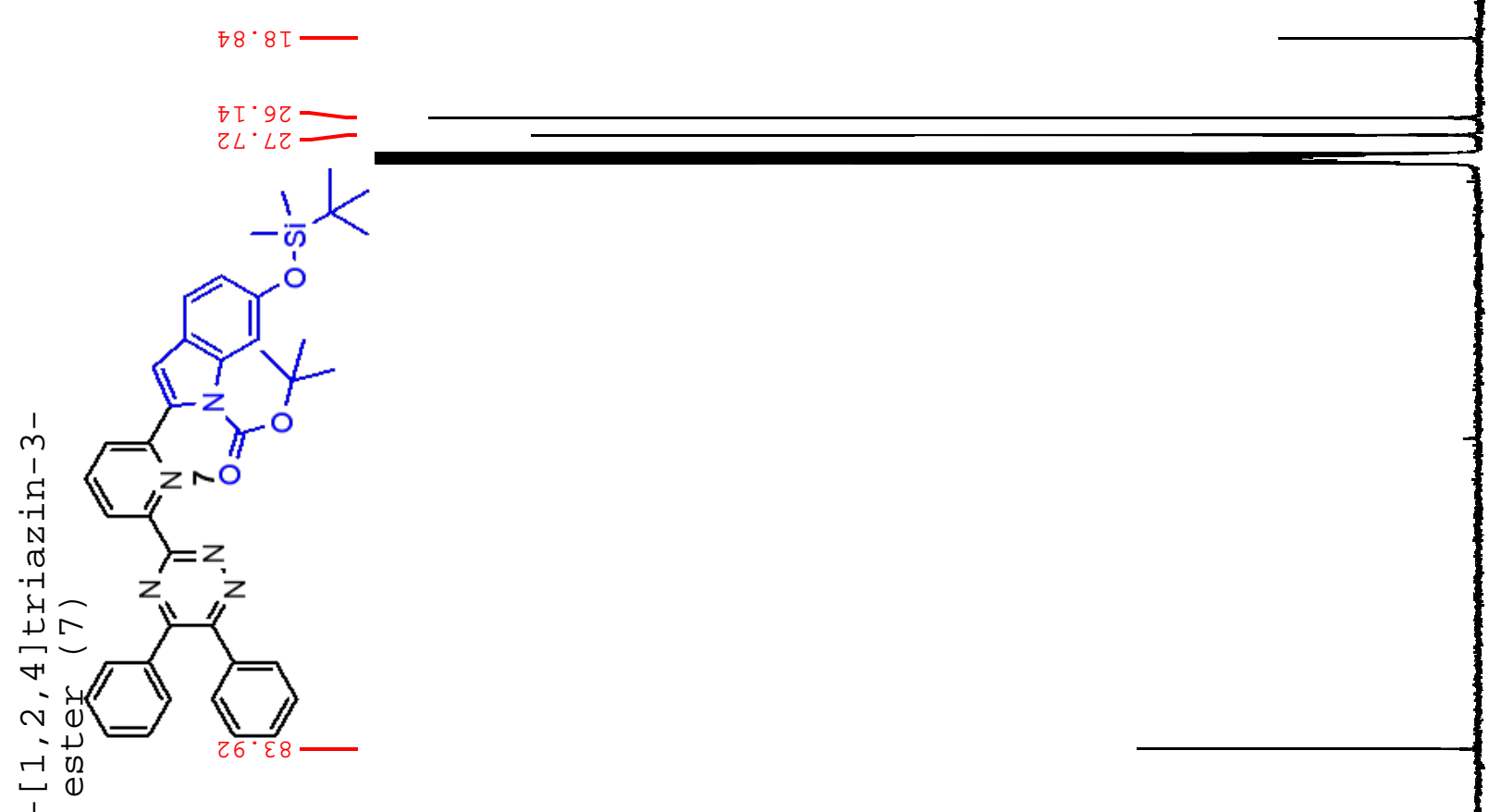

1

닺다

들ำ

응 응

담ำ

인

ט.

1)

1.

๑

10

'ं.

$\stackrel{2}{2}$

츠

$\times 0$

근

¿

สิ 1

가 가

ज ब

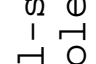

츠읃

푸다

(1)

हत्तन

긍 곡

में

능

6 כ

귕다

थ '

(1) 는

거

$p+1$

(2)

I'

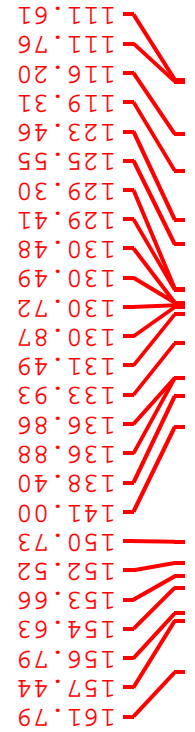




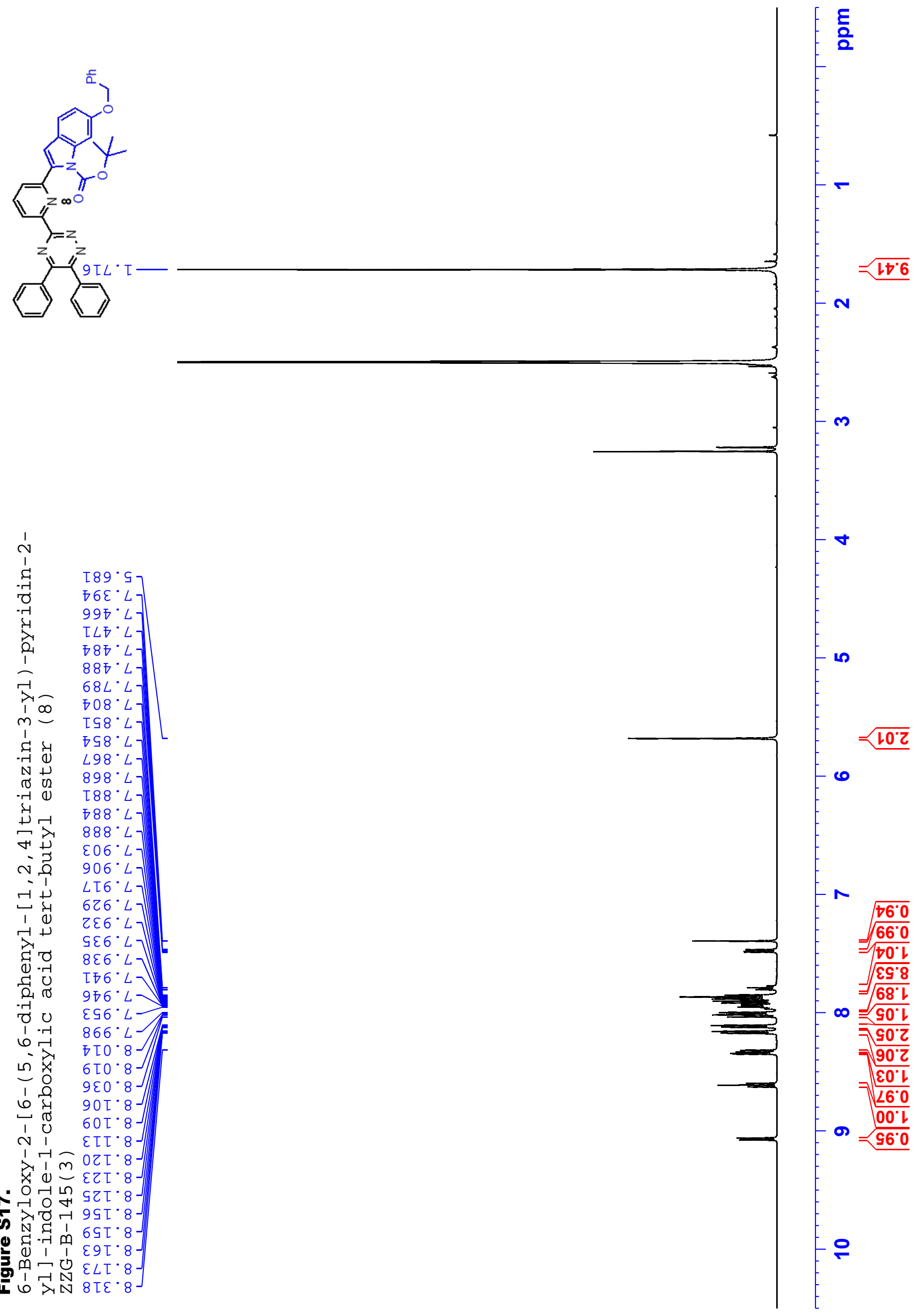




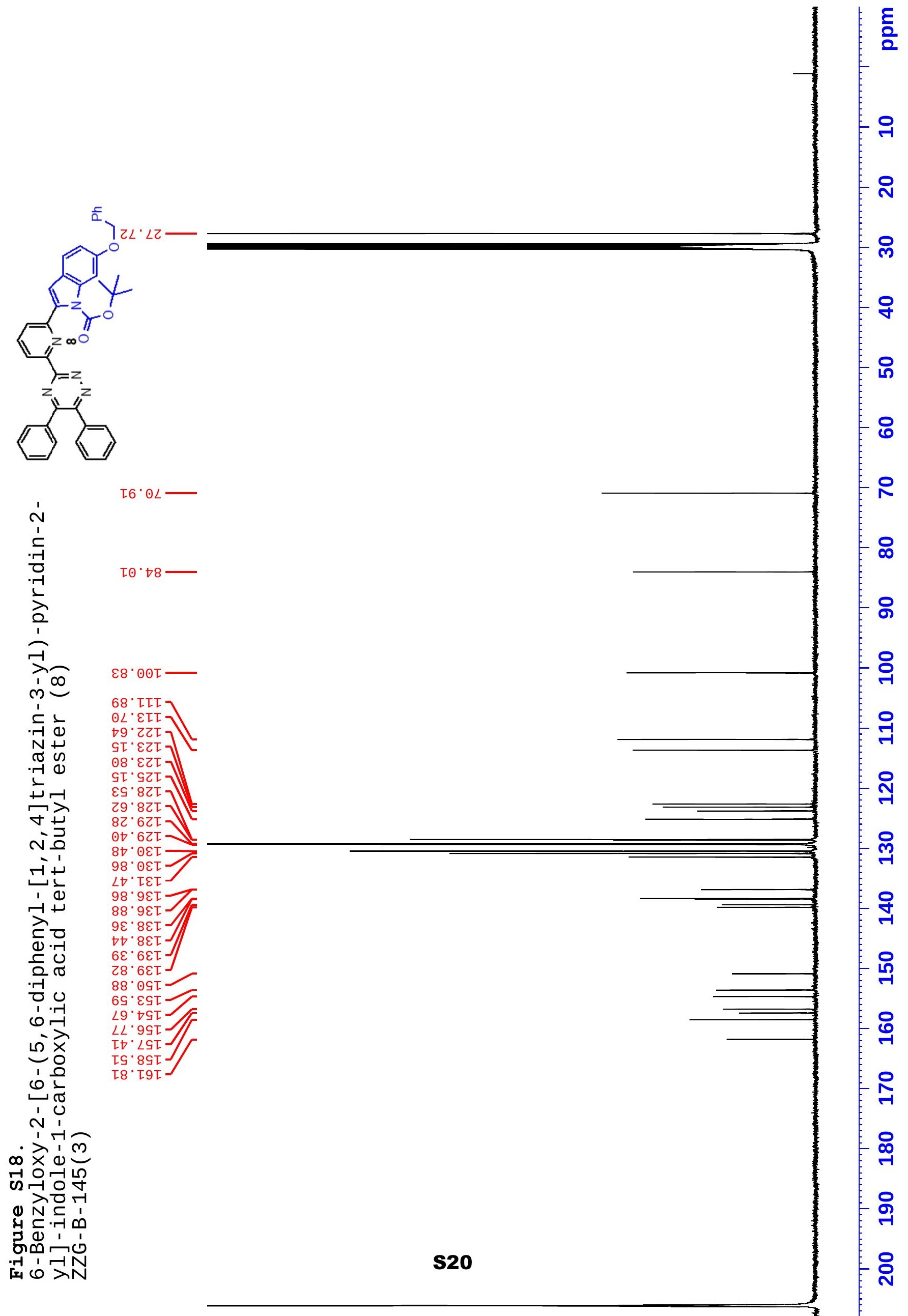



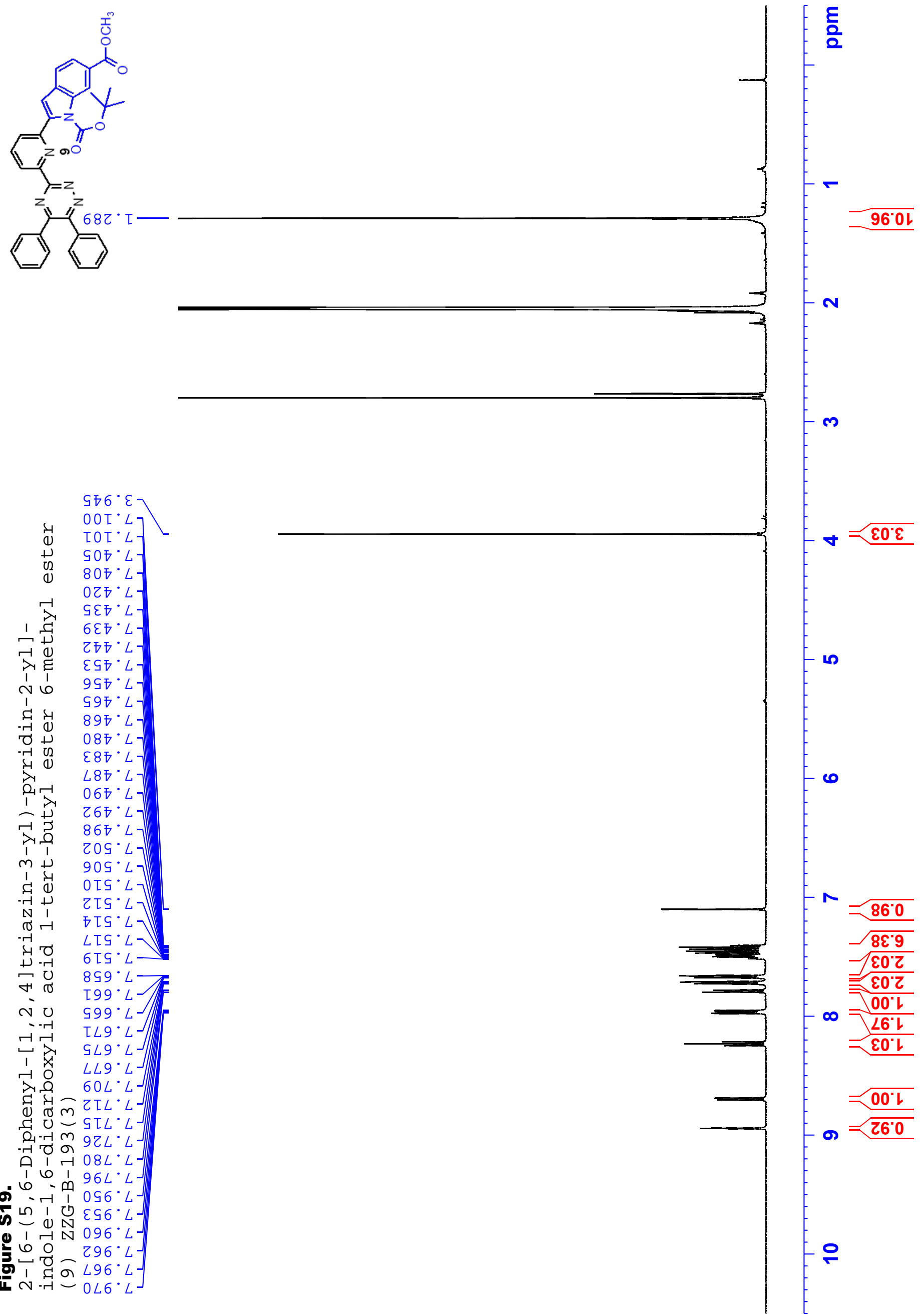


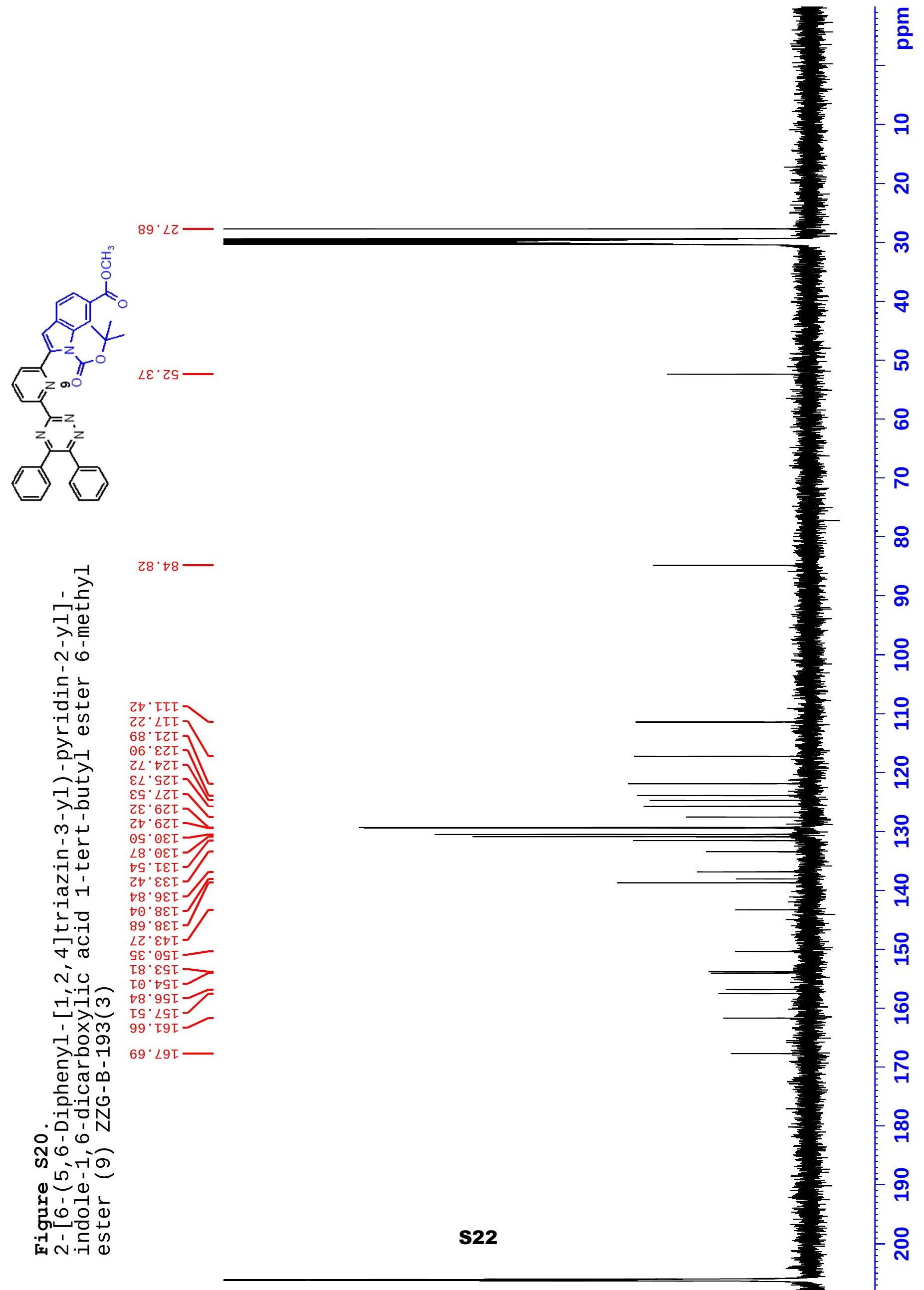



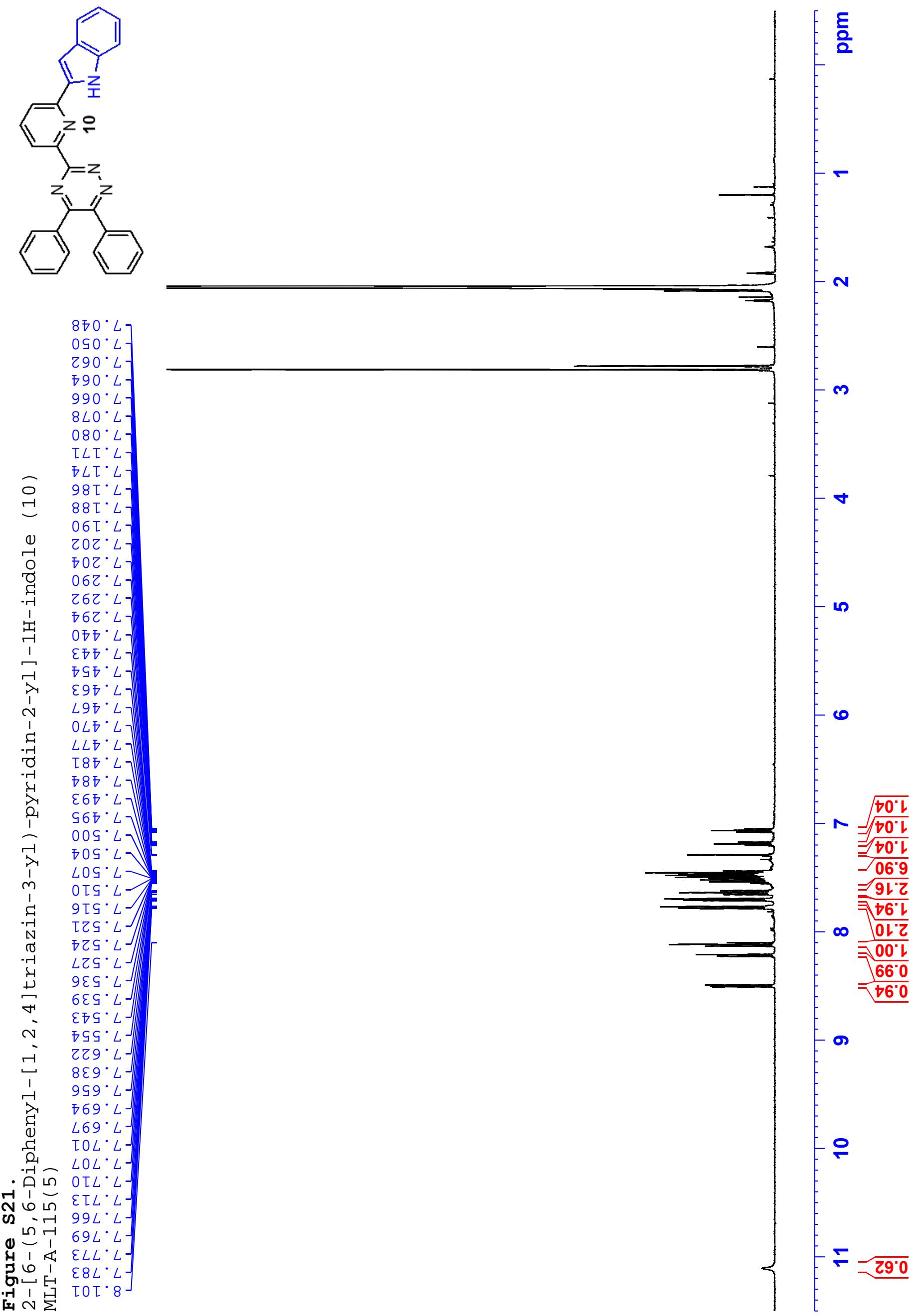


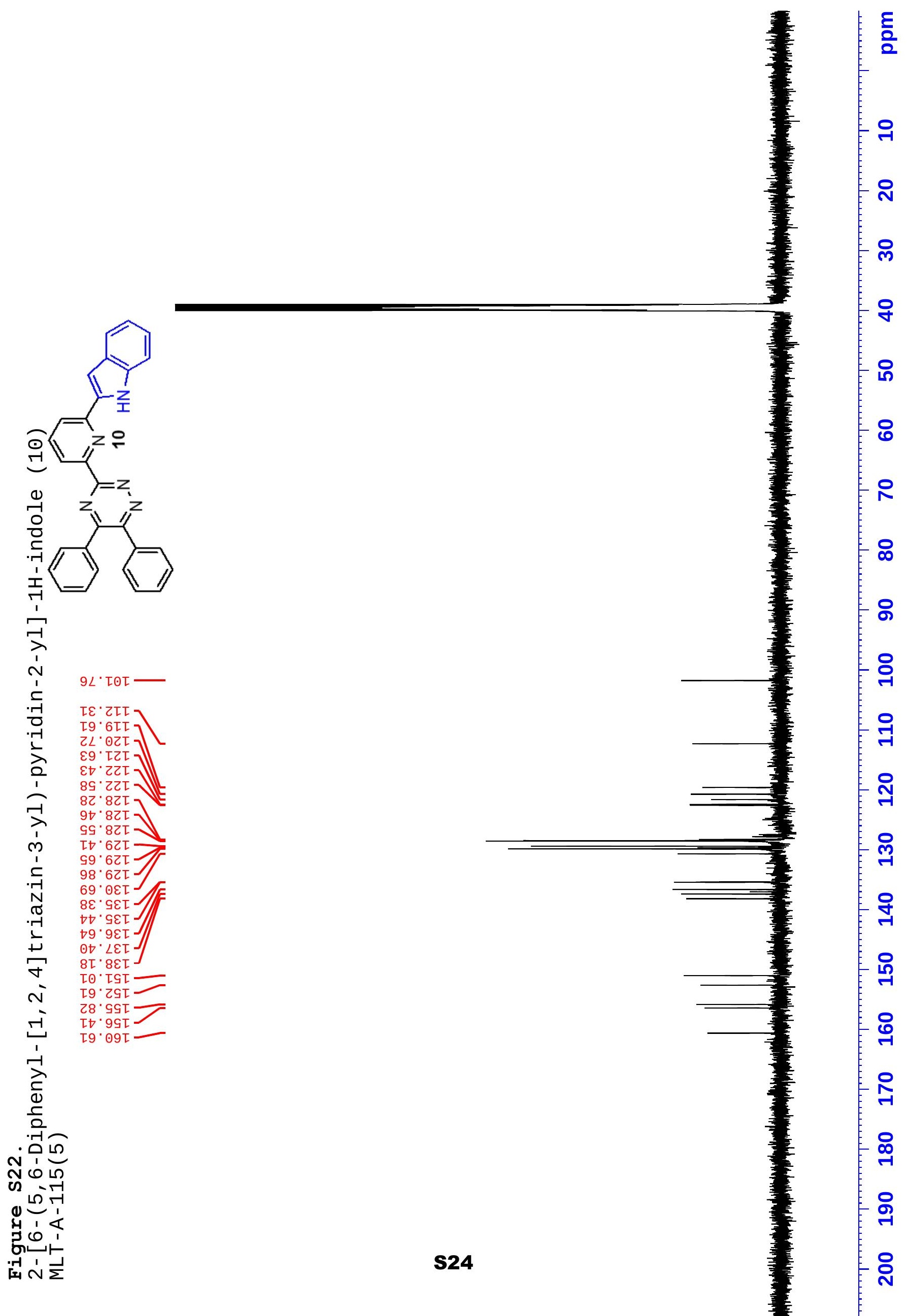



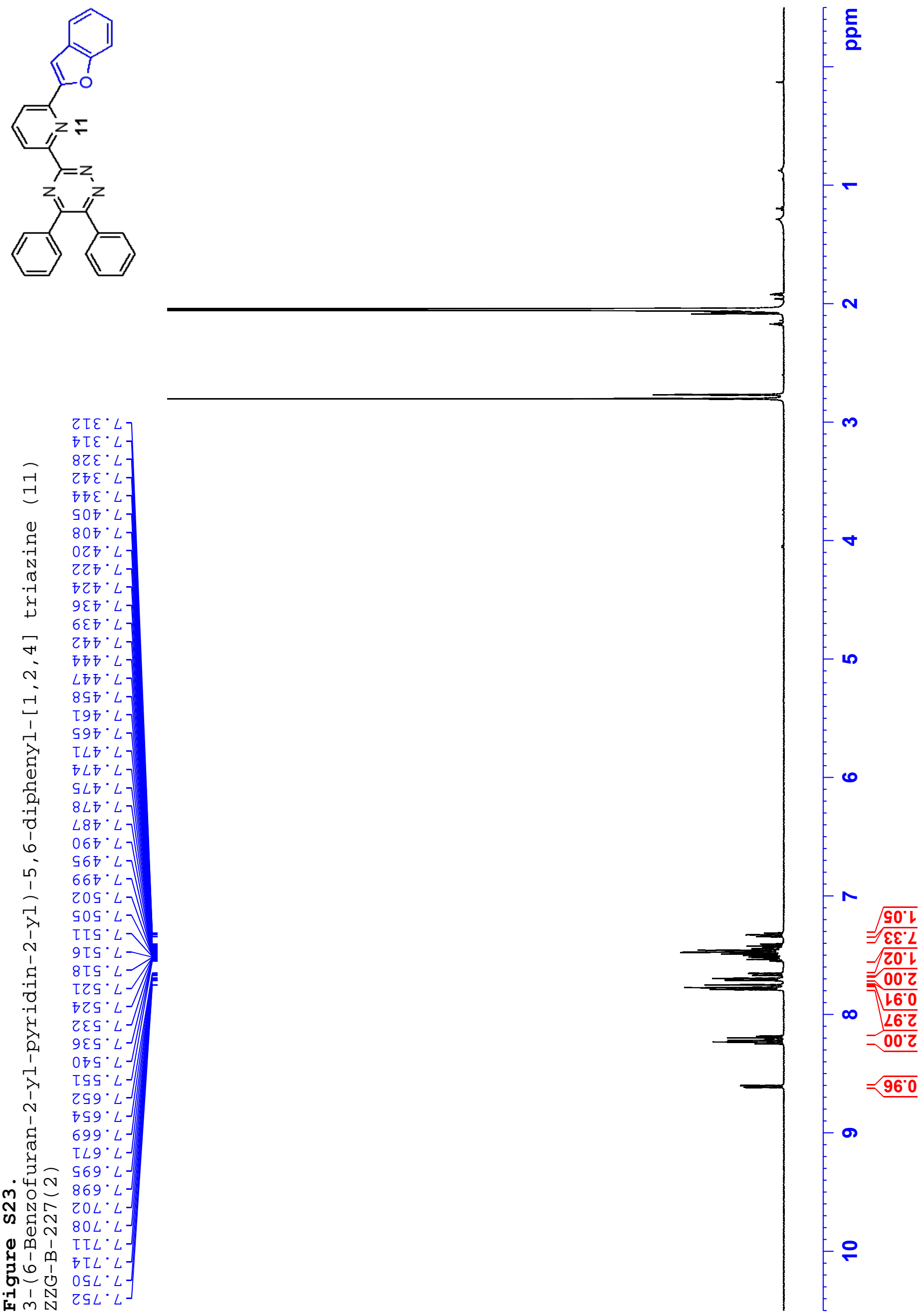


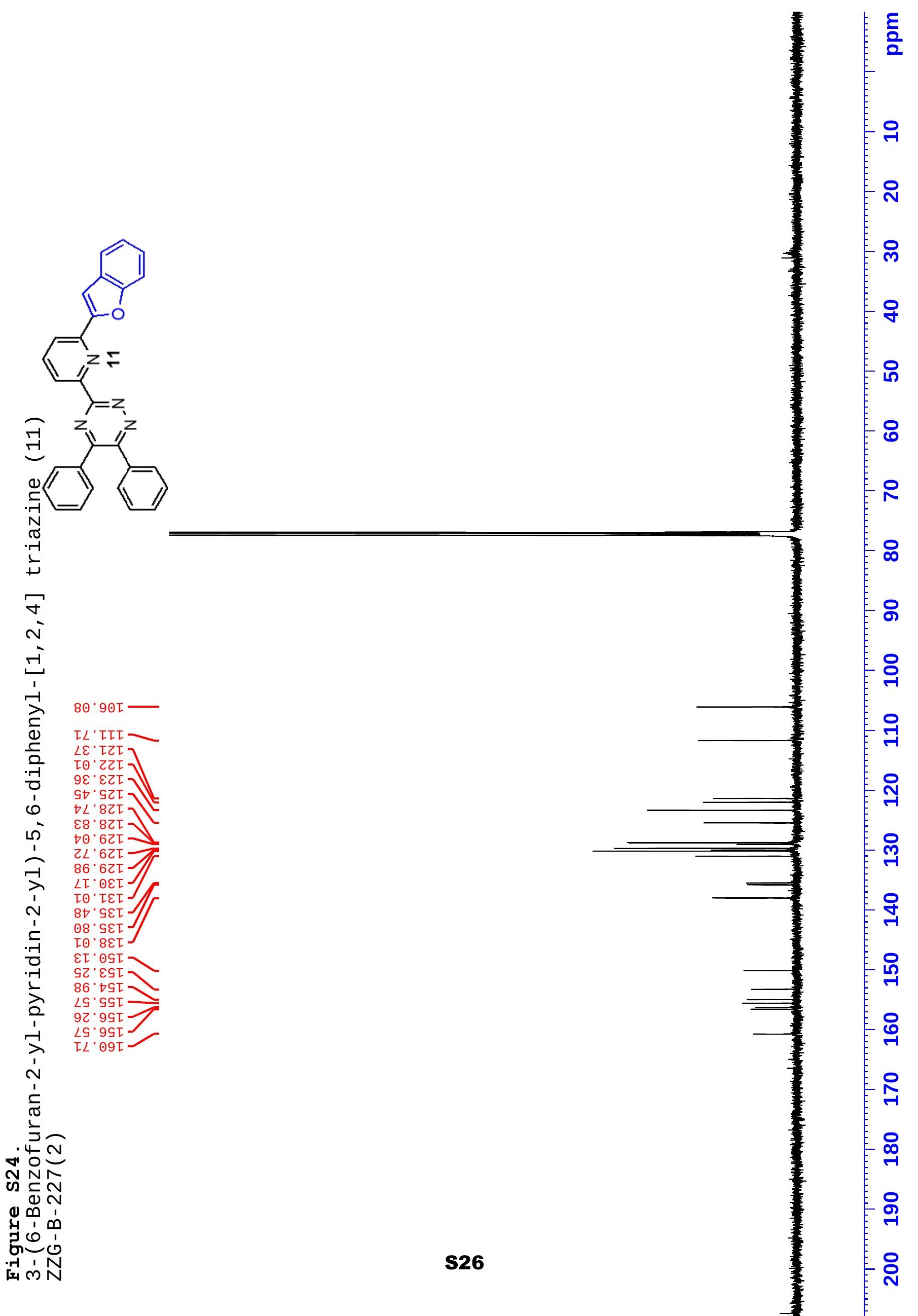




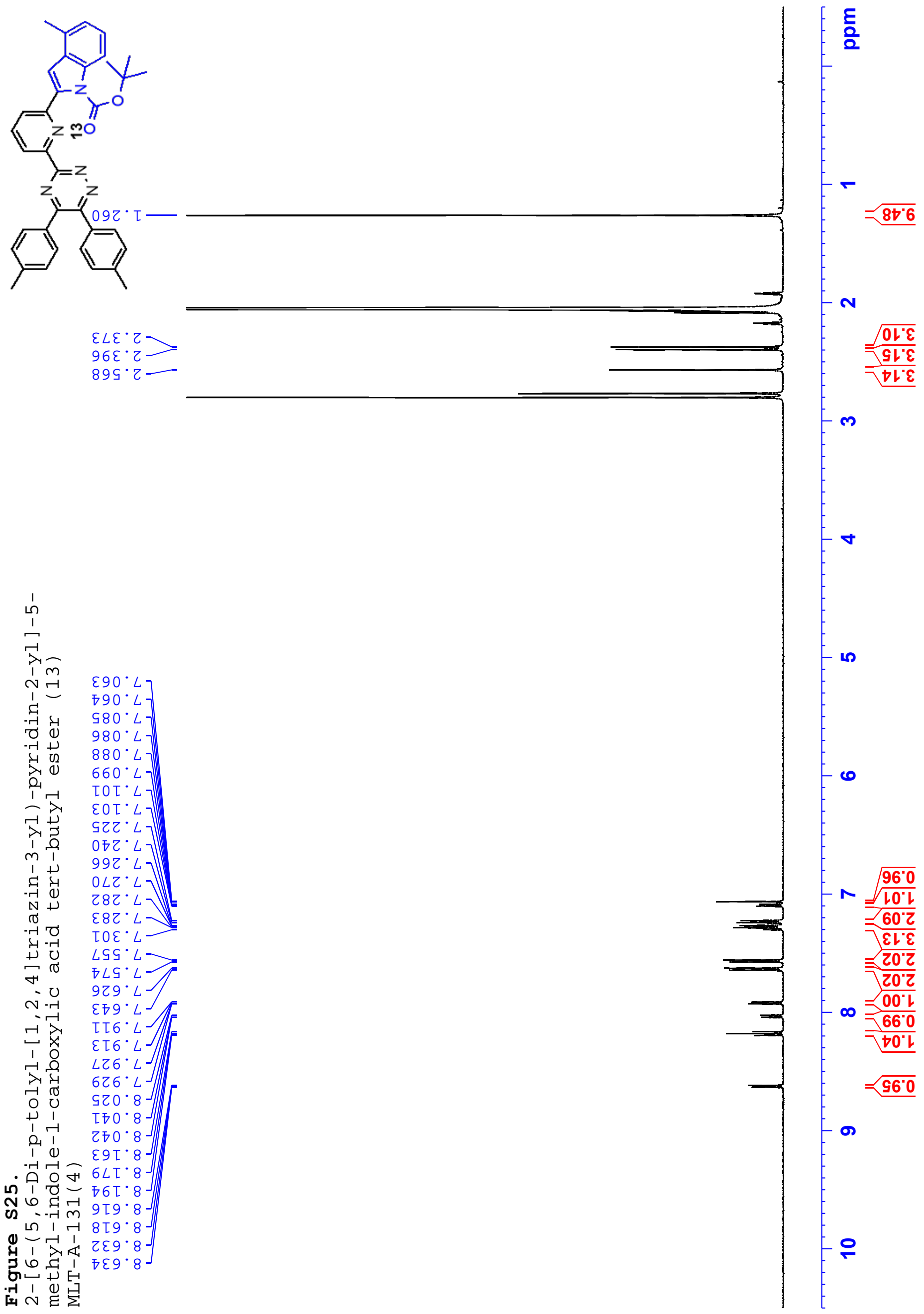




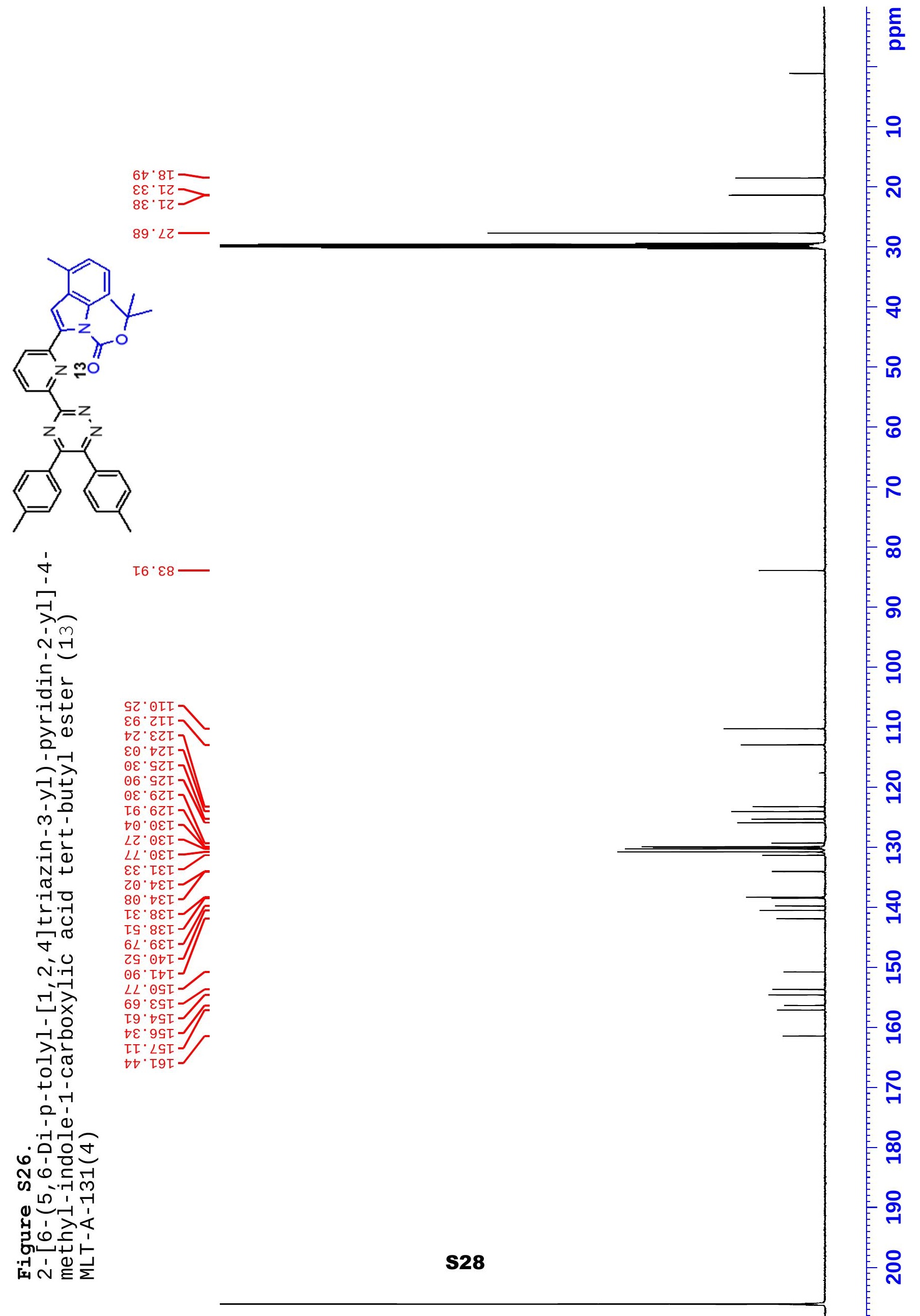




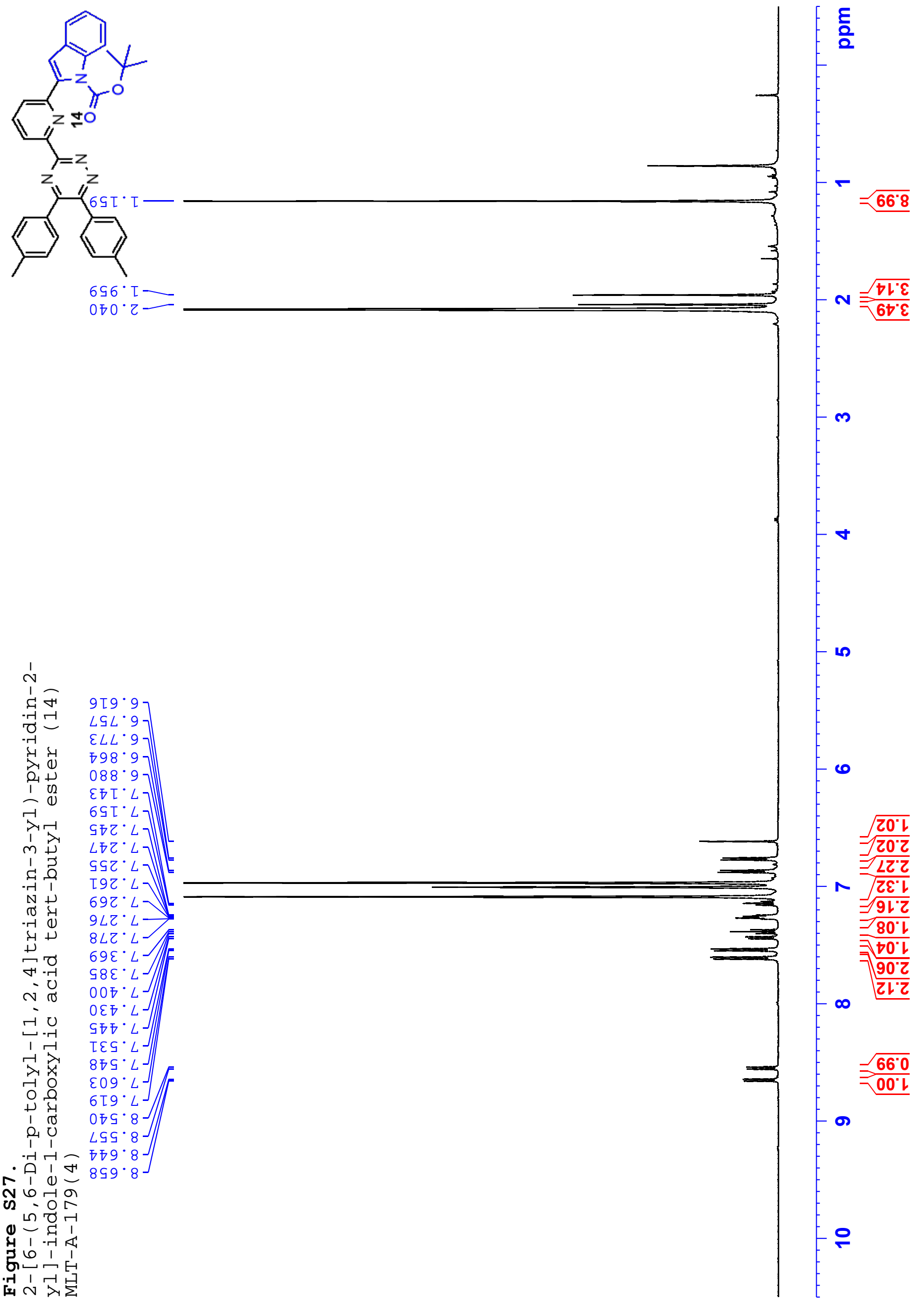




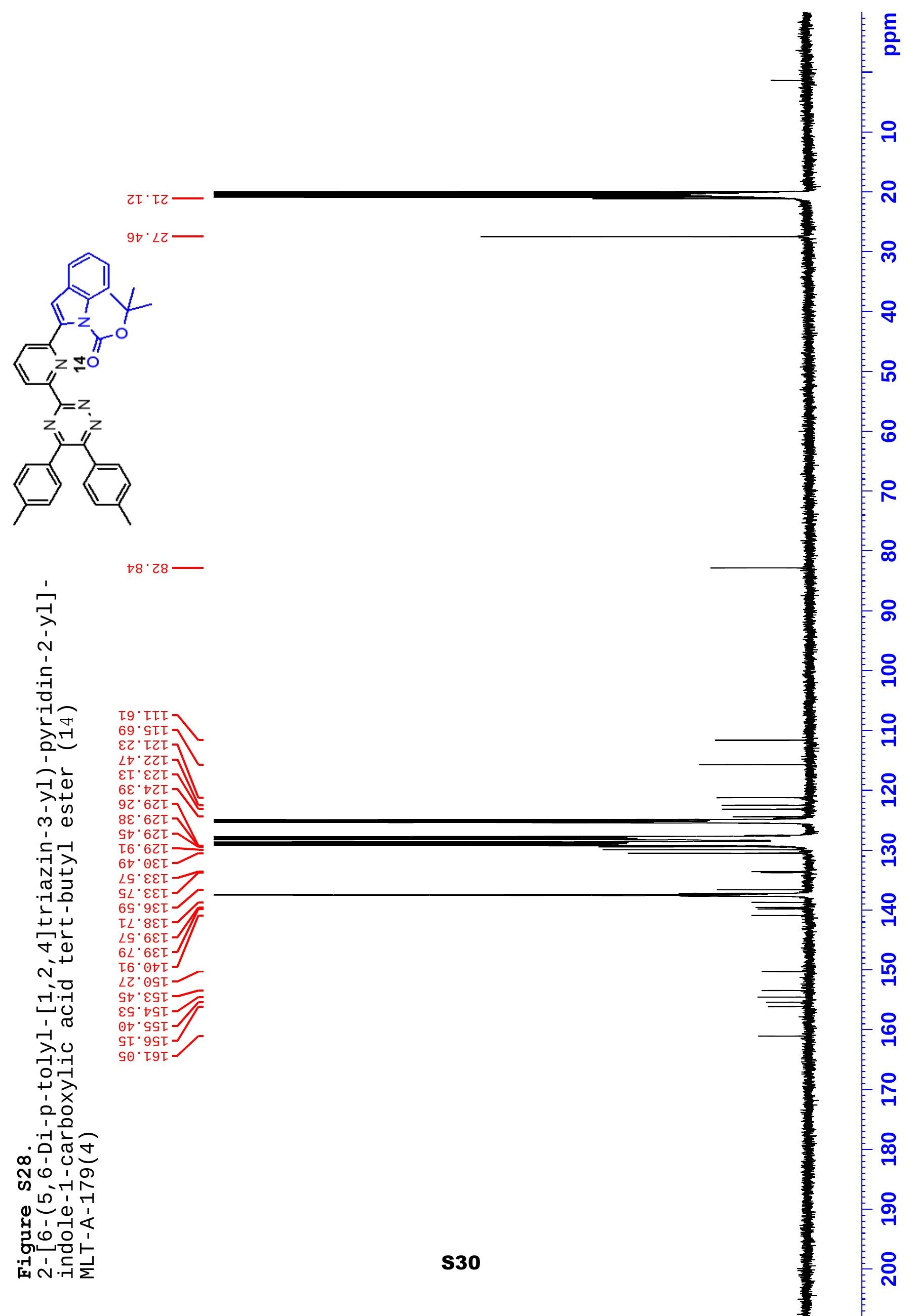




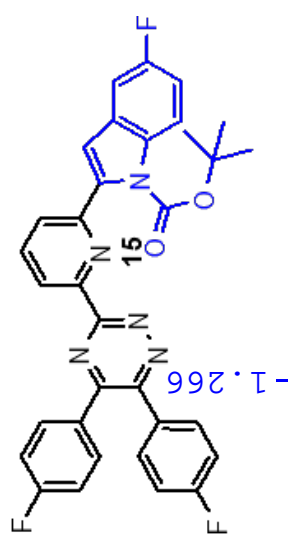

$\stackrel{1}{2}$

. त्व

.न

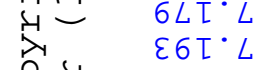

은

다 602 .

तथ $9 \tau 2$.

मेन LZZ'L

टत $\quad s t z \cdot L$

글 $\quad$ ㄷ․ $L$

त ' 69ट $L$

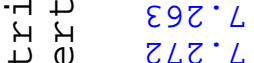

$\pm 0$

$\nabla$

․ㅜㅇ

$\left.9 \angle Z^{\circ} \angle\right]$

$60 t^{\circ} L$

तं

Zहt: $\backslash$

I. U IZL $L$

GZL'

IEL.

$8 \varepsilon L ' L$

St $L \cdot L$

$6 \nabla L \cdot L$

$\nabla L L=L$

$6 \angle L \cdot L J$

$m$

$N$

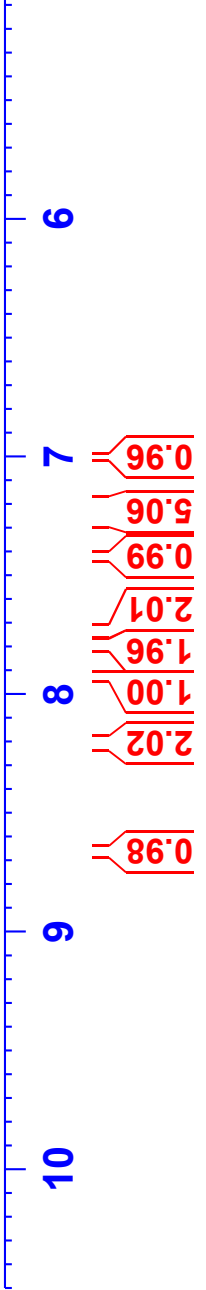




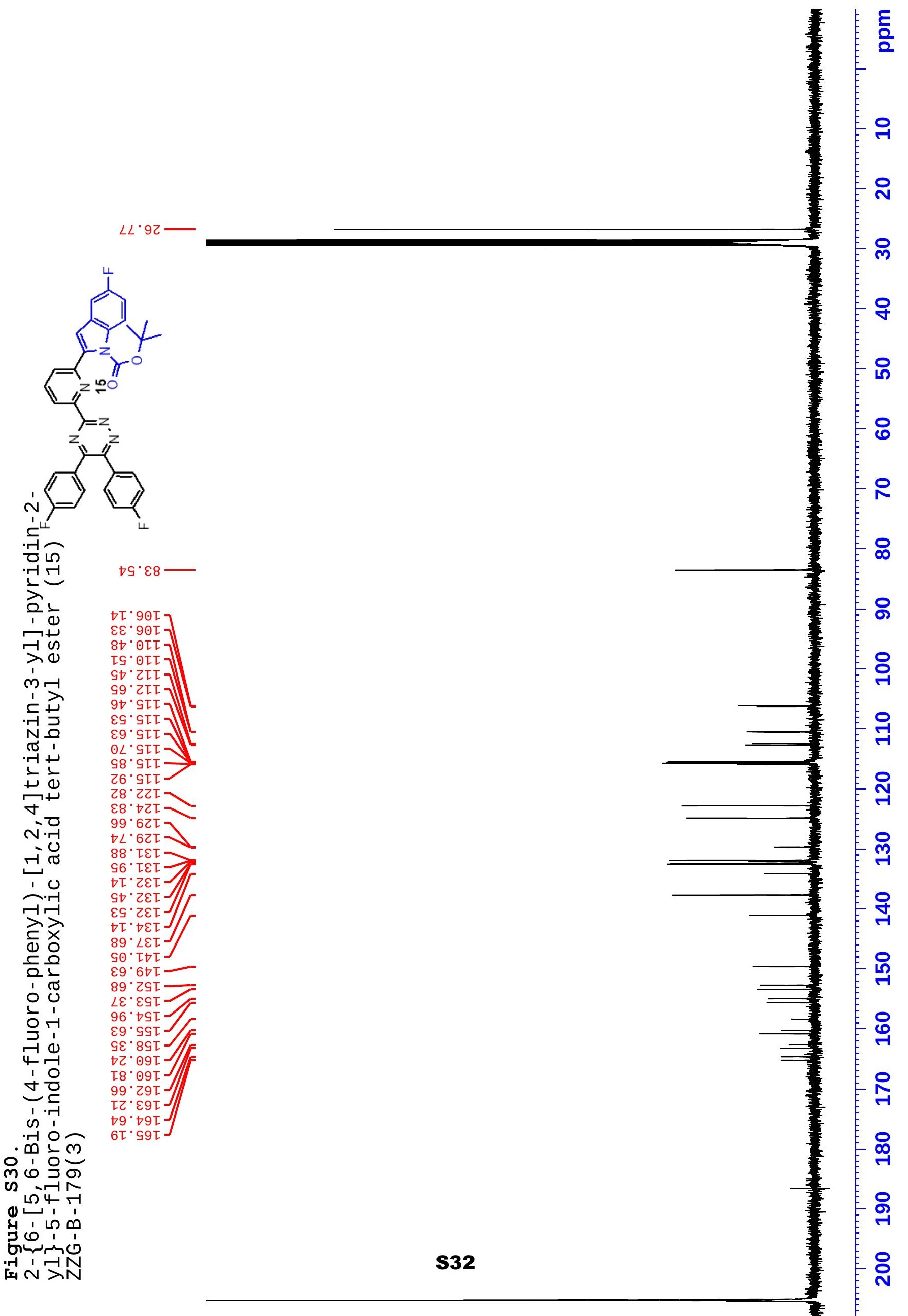




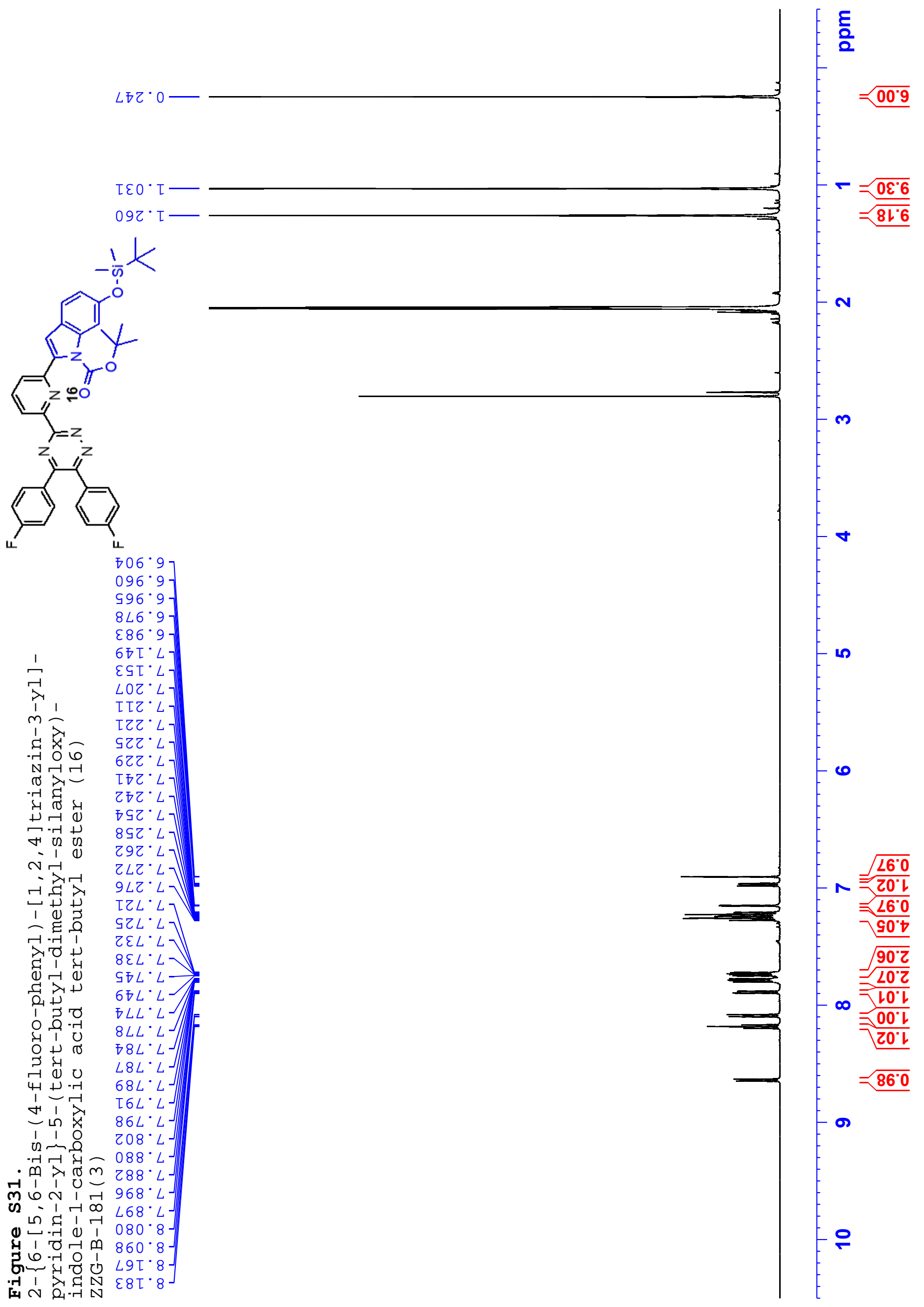




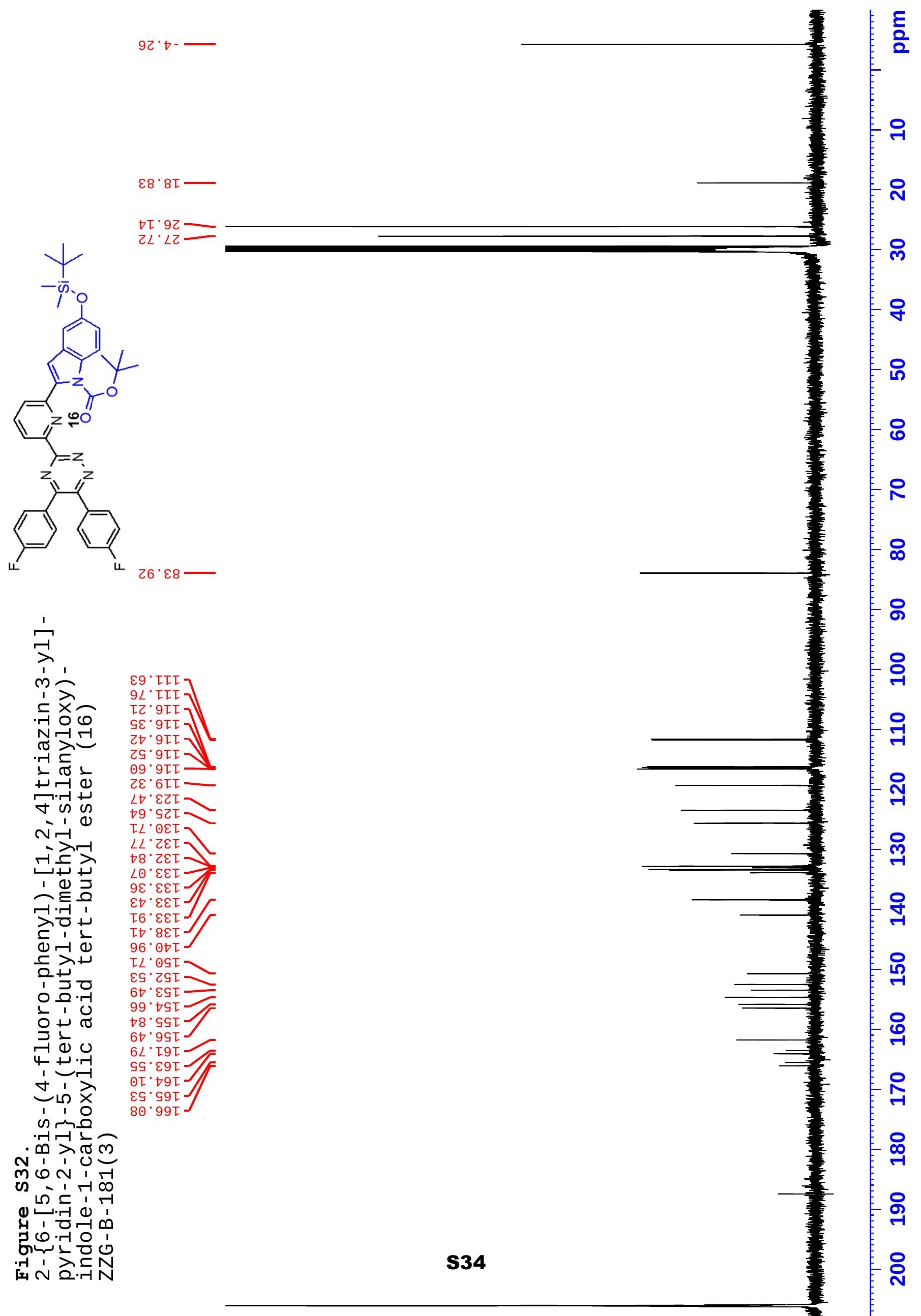




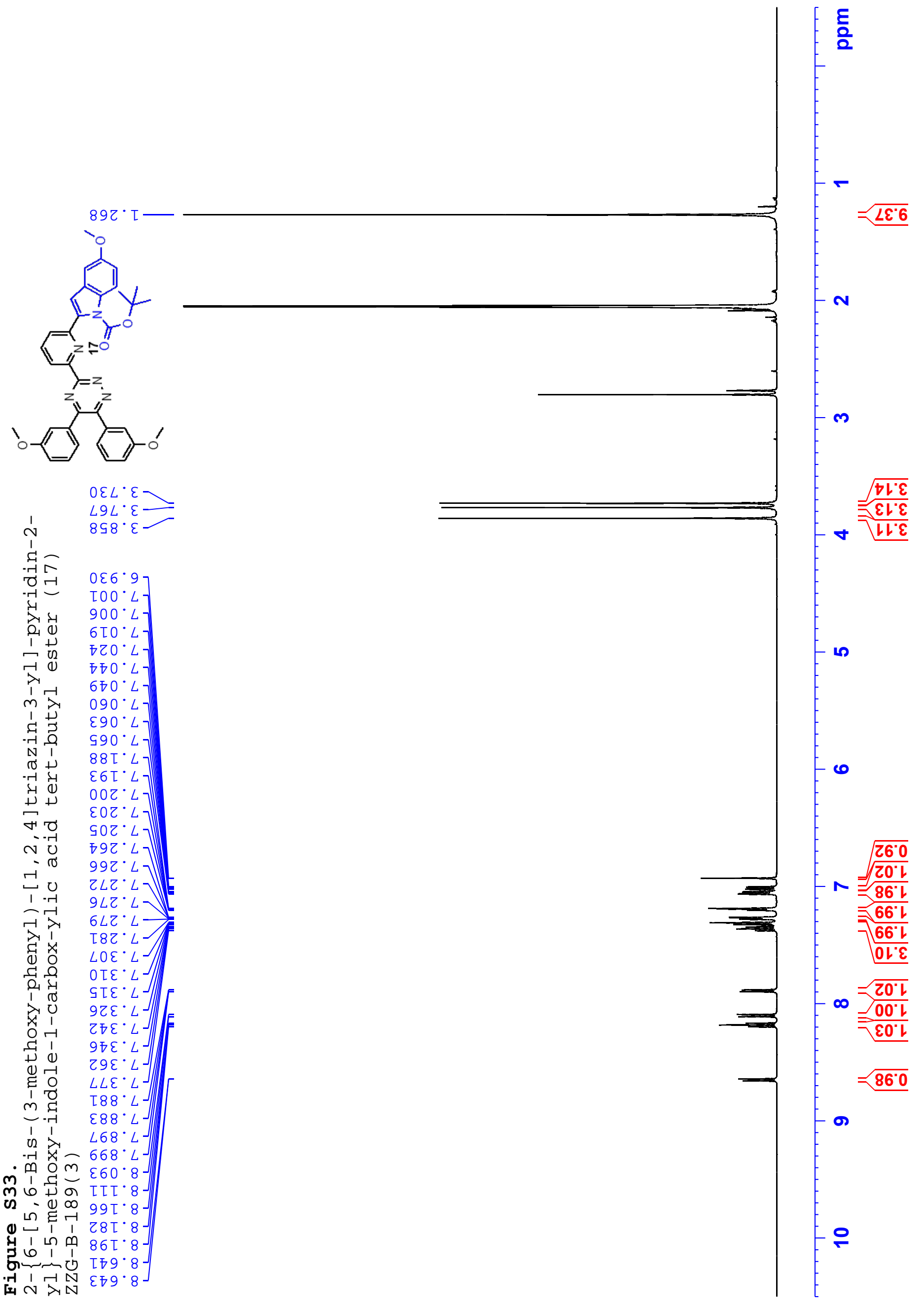



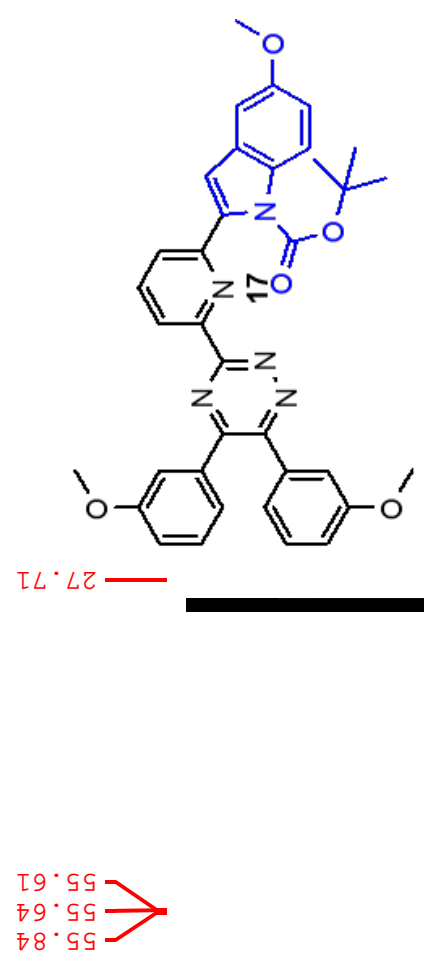

IL' $L Z=$

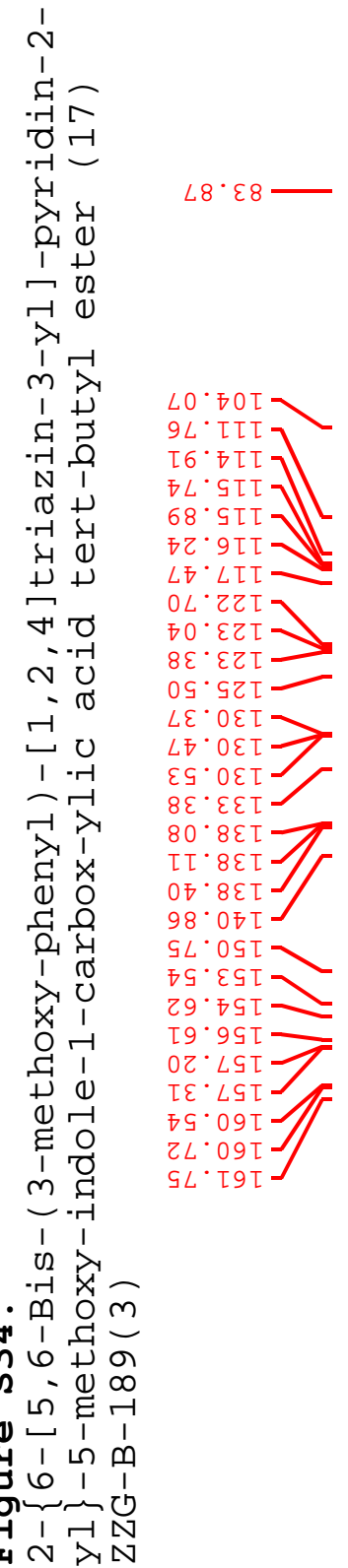




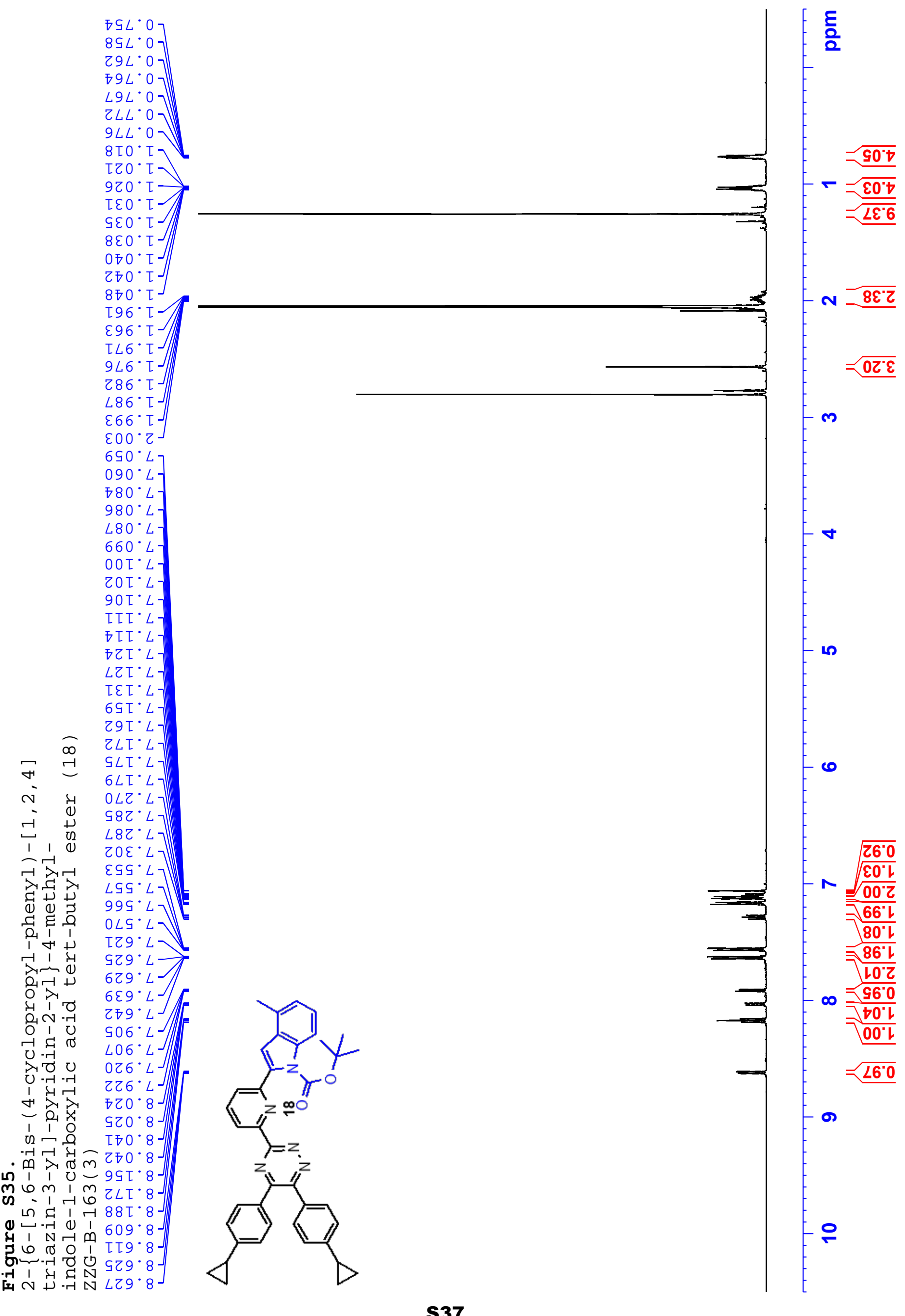




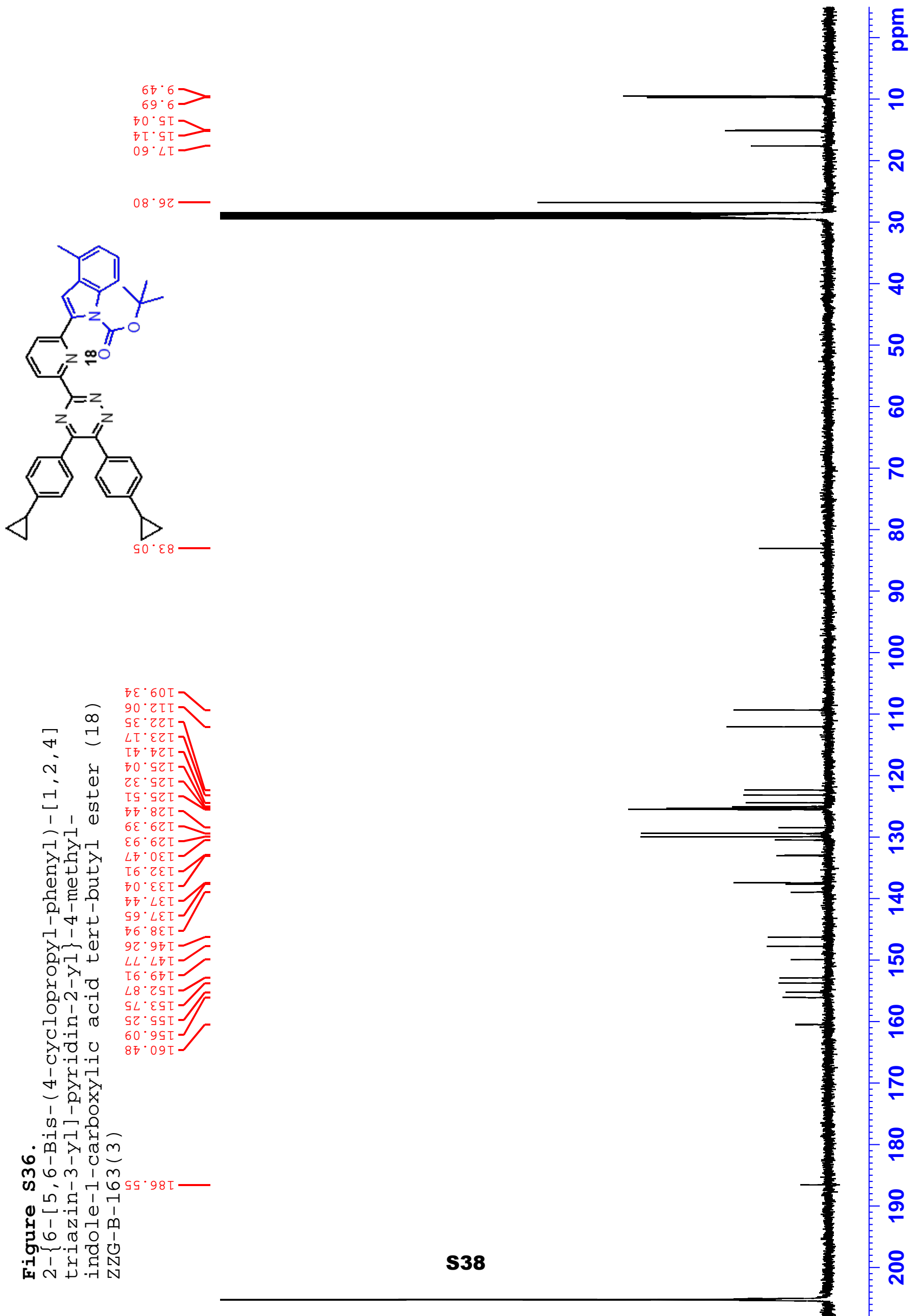


$9 \angle L \cdot 07$

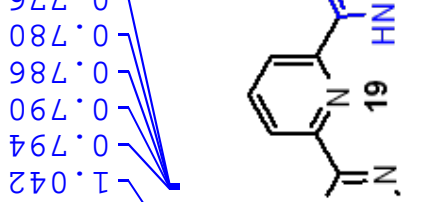

$2 \downarrow 0 \cdot I$

$9+0 \cdot \tau$

$650^{\circ} \cdot \tau$

$290^{\circ} \mathrm{L}$

$990 \cdot \tau$

$296^{\circ} \mathrm{I}$

GL6" $\mathrm{T}$

$986^{\circ} \mathrm{I}$

$766^{\circ} \tau-$

โ०० $冗$

โLO 乙

8[0.乙

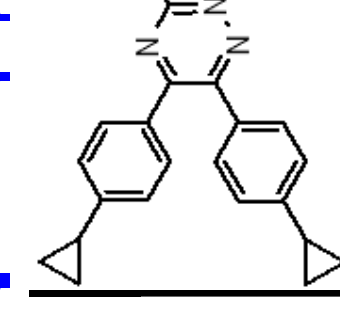

$S t 0^{\circ} L$

$\angle \nabla 0^{\circ} \angle-$

$690^{\circ} \mathrm{L}$

โ90. L

$\varepsilon 90^{\circ} L$

$\angle L 0^{\circ} \angle$

m $8 D I$.

c t9L.

.

†8I' $L$

$\angle 8 I \cdot L$

LOZ '

$\checkmark \quad 8 \angle Z^{\circ} L$

ते ह89.

ت단

$\overbrace{}^{\prime}$ L69.

글

高

음요

요 [G9. L

응긍 $989^{\circ} L$

으응 $689^{\circ}$

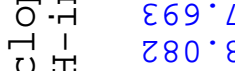

ग्न

Uं हII'8

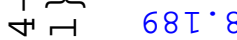

ป

ज ज०२ 8

.

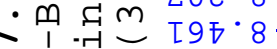

m 0 ठ

થ 건

0 元' $8 \angle t^{\prime} 8$

4 '

$90 I \cdot I$
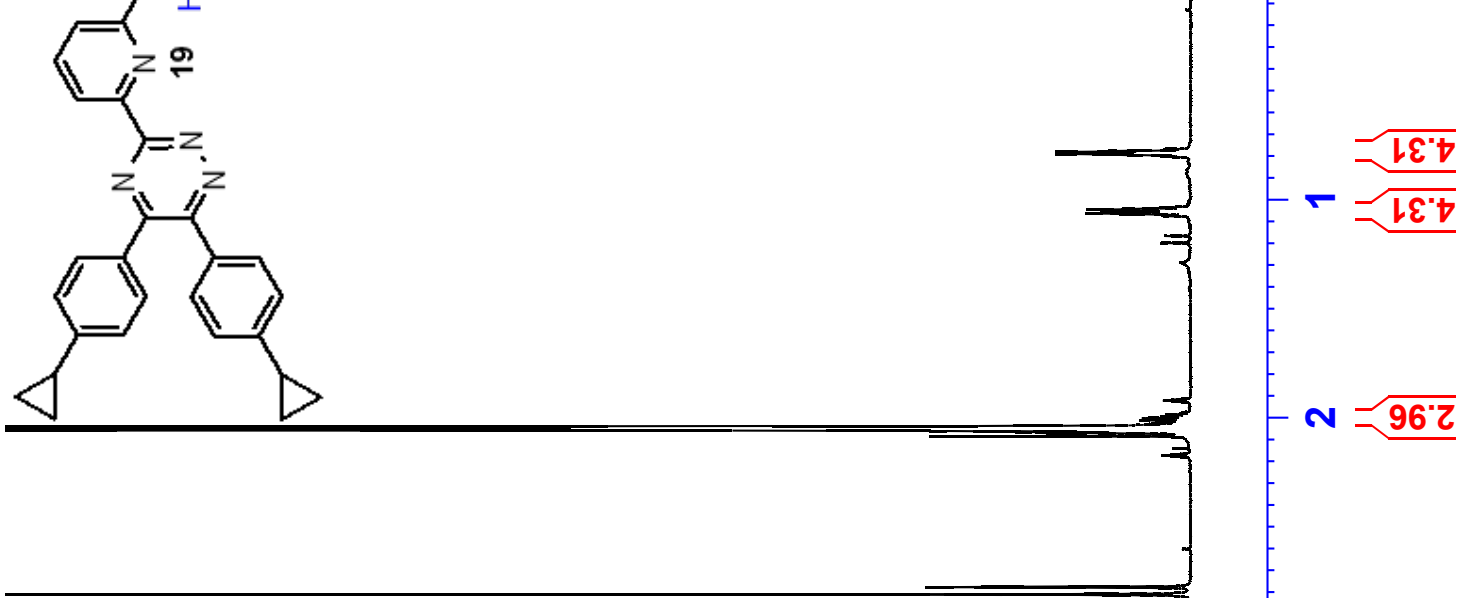

$N=\longdiv { 9 6 \cdot z }$

m

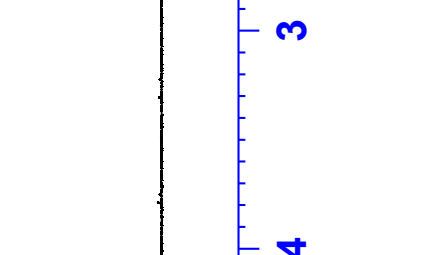

0.

घ $\sim \mathcal{N}$ 


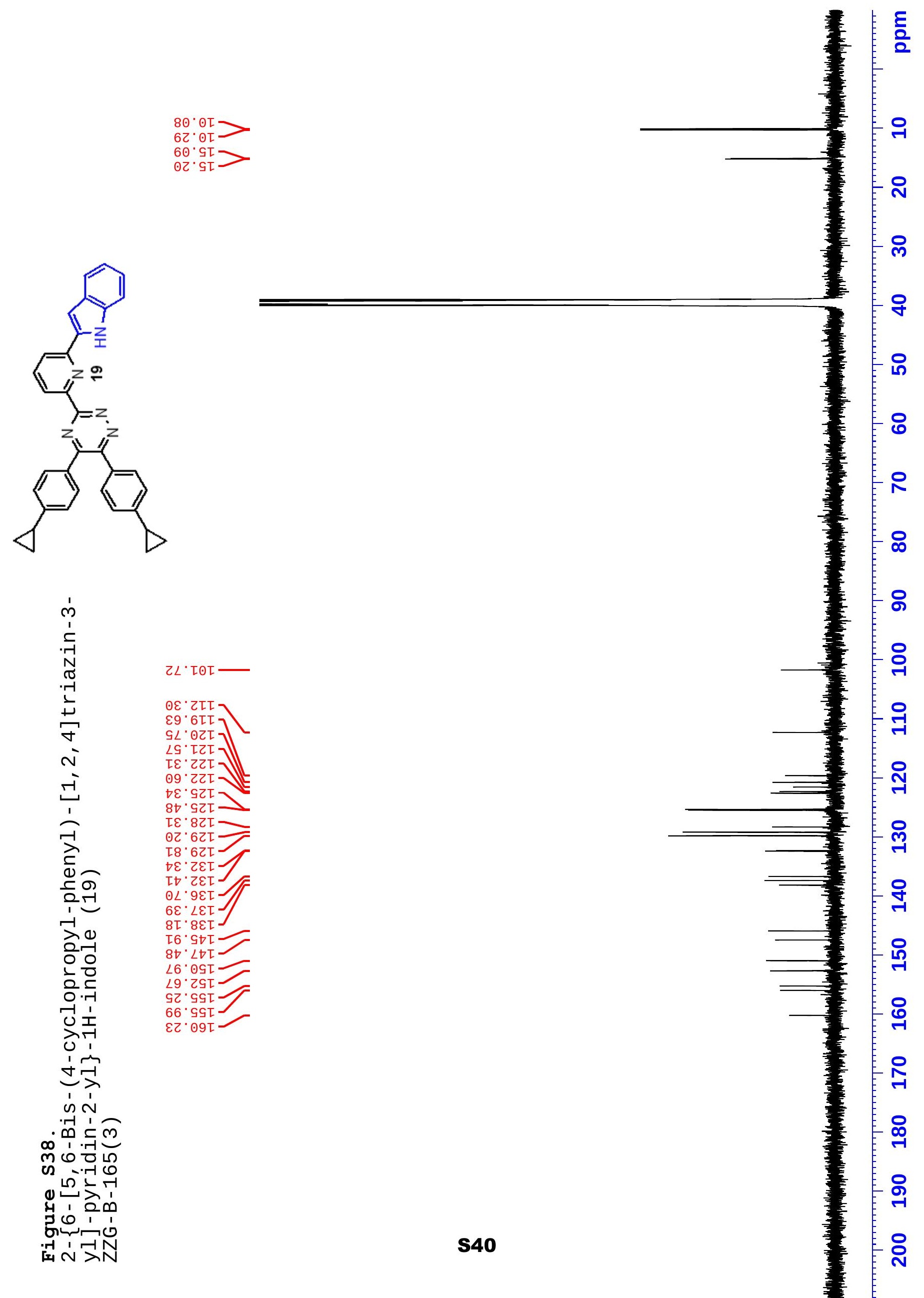




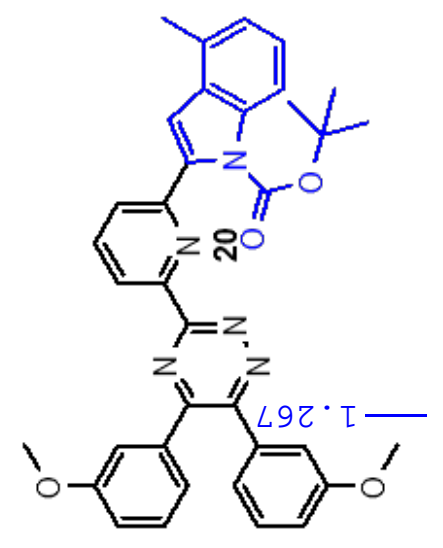

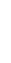

政
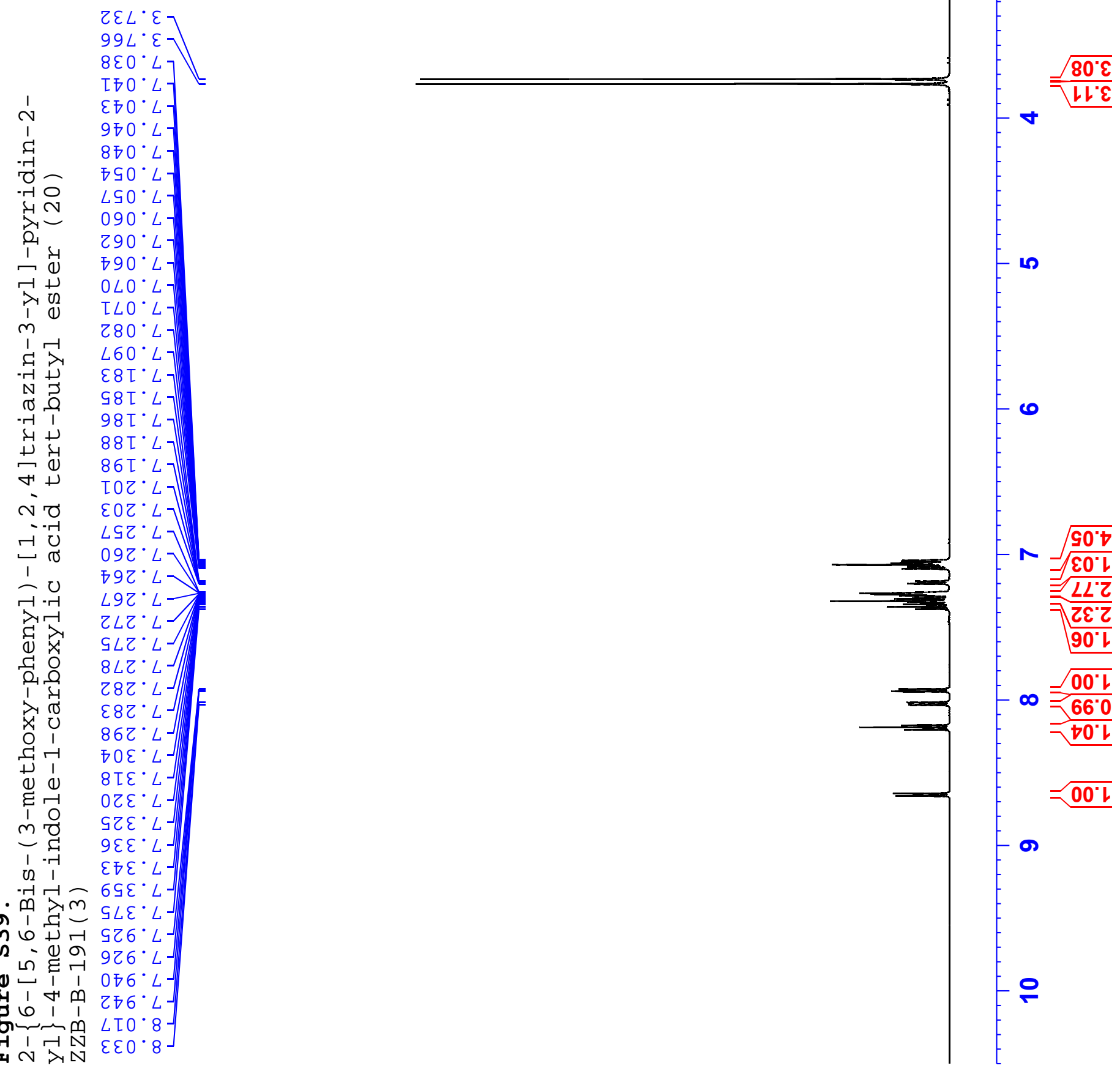


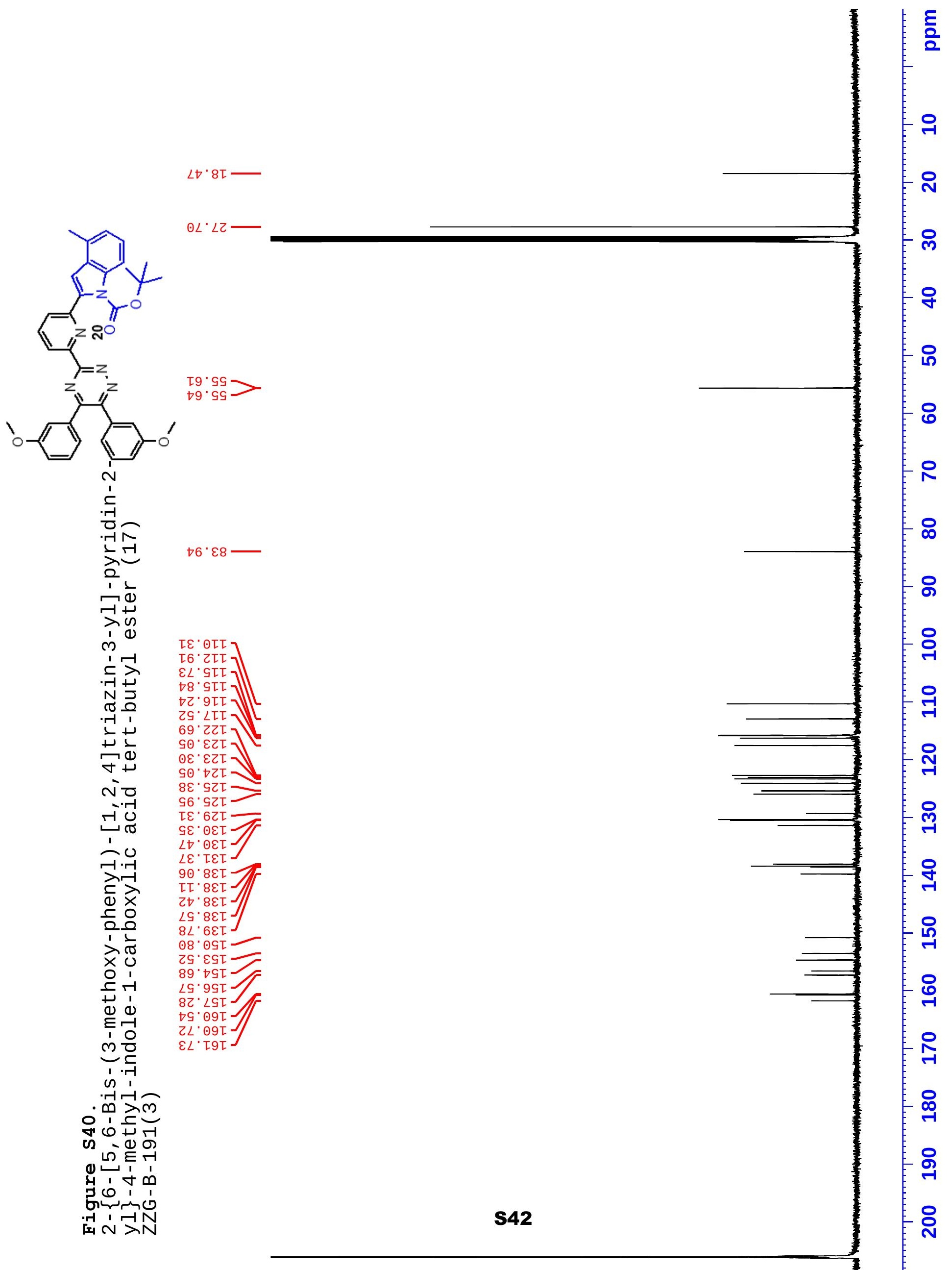



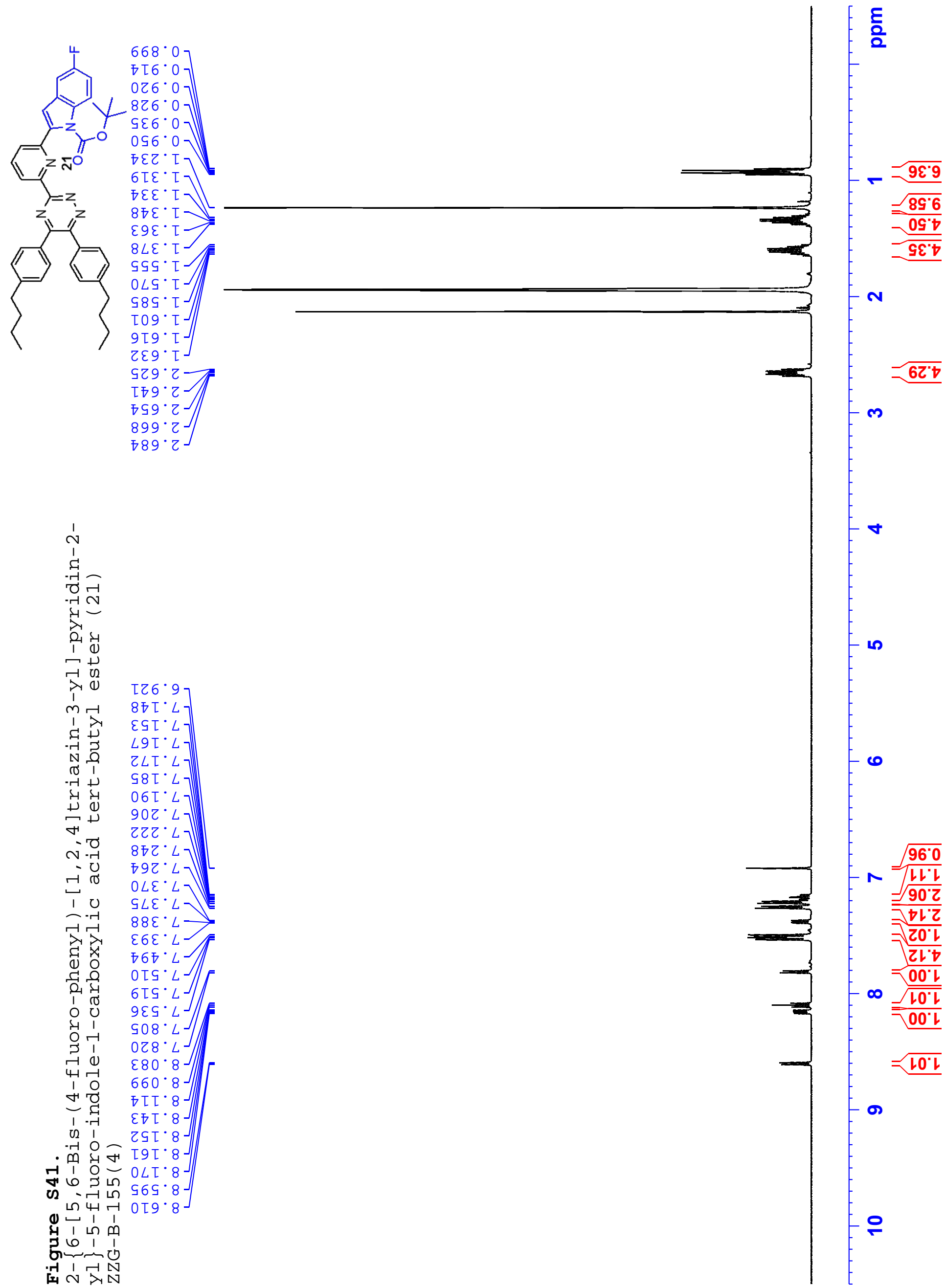


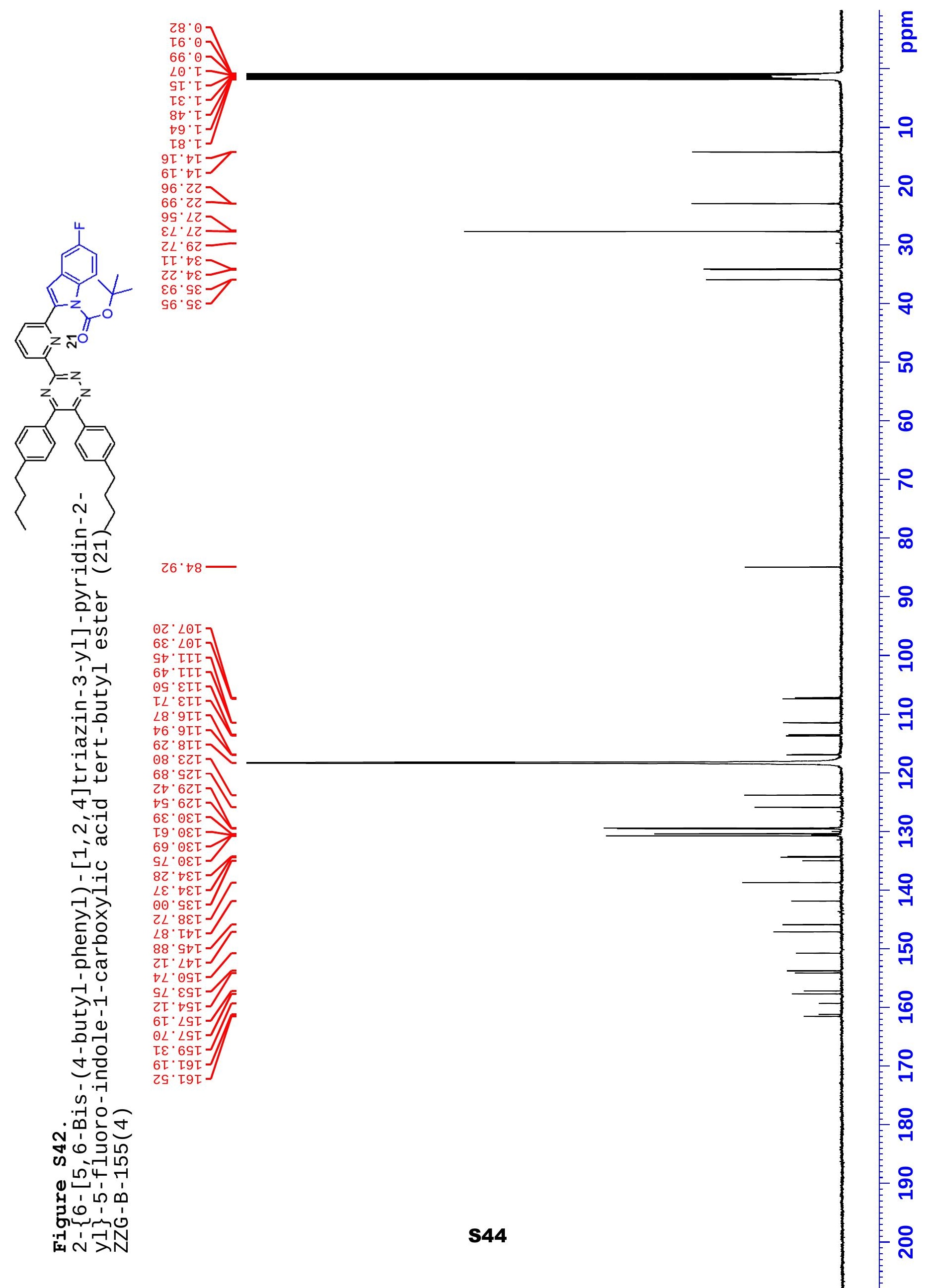




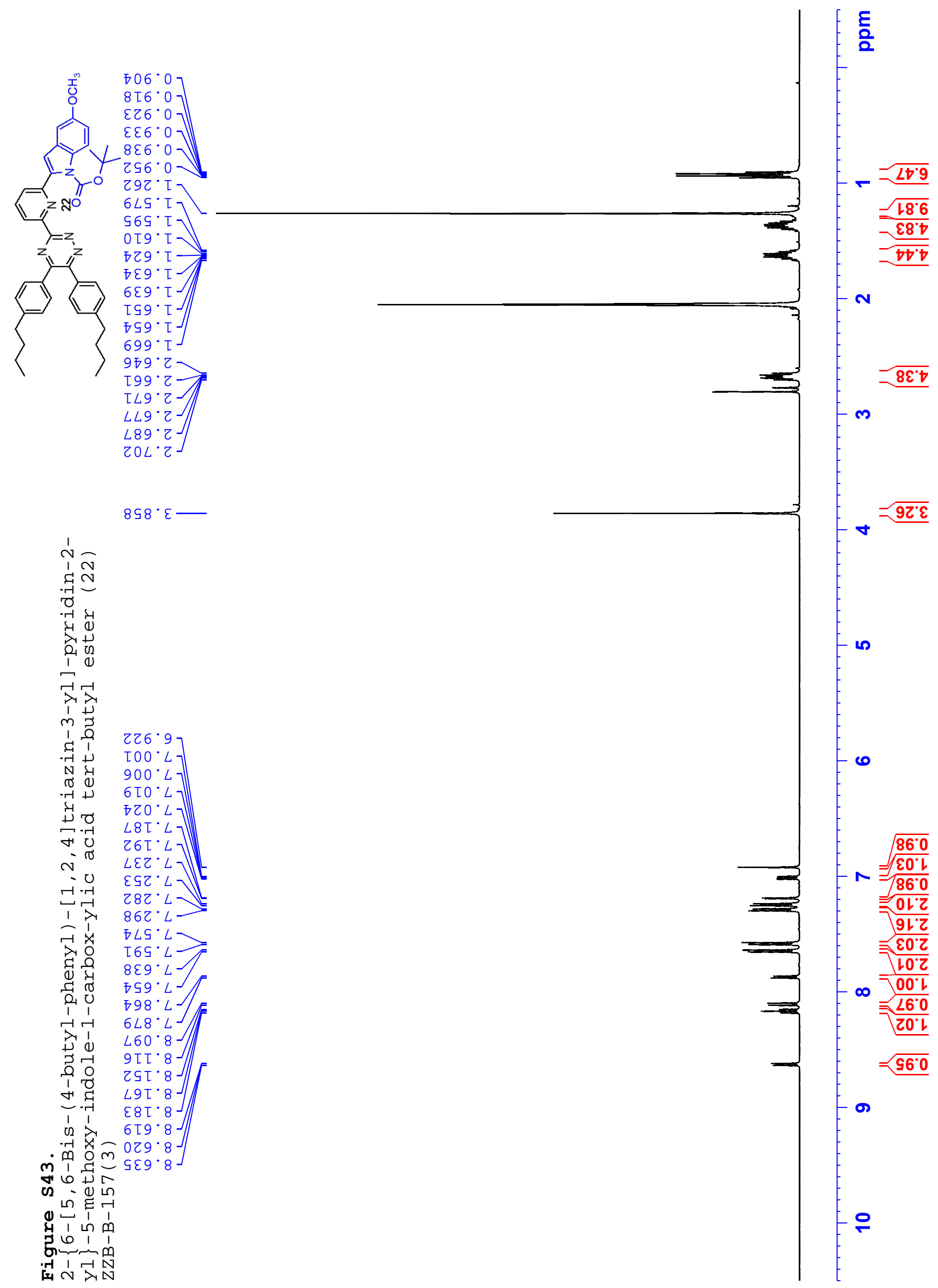




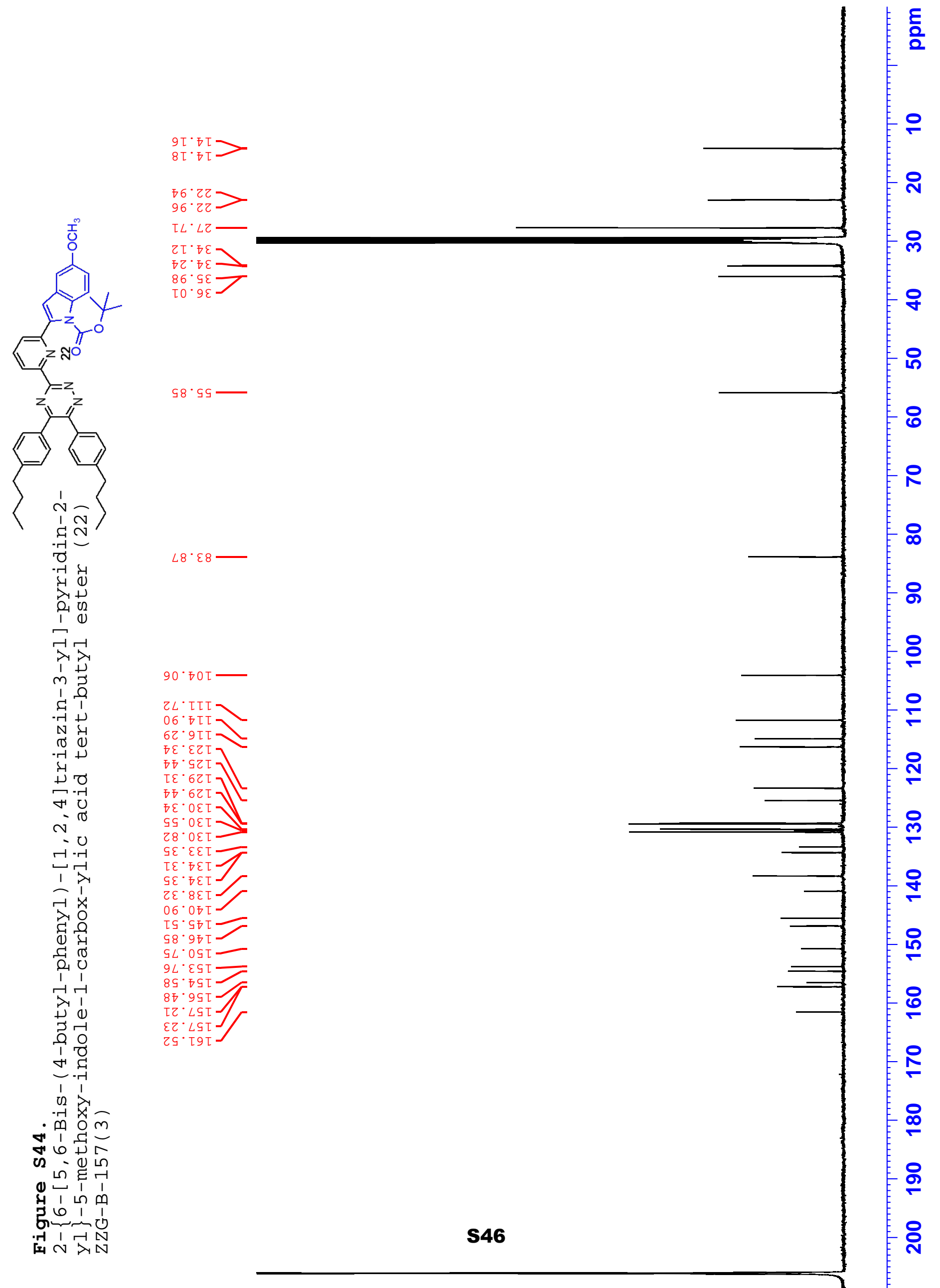




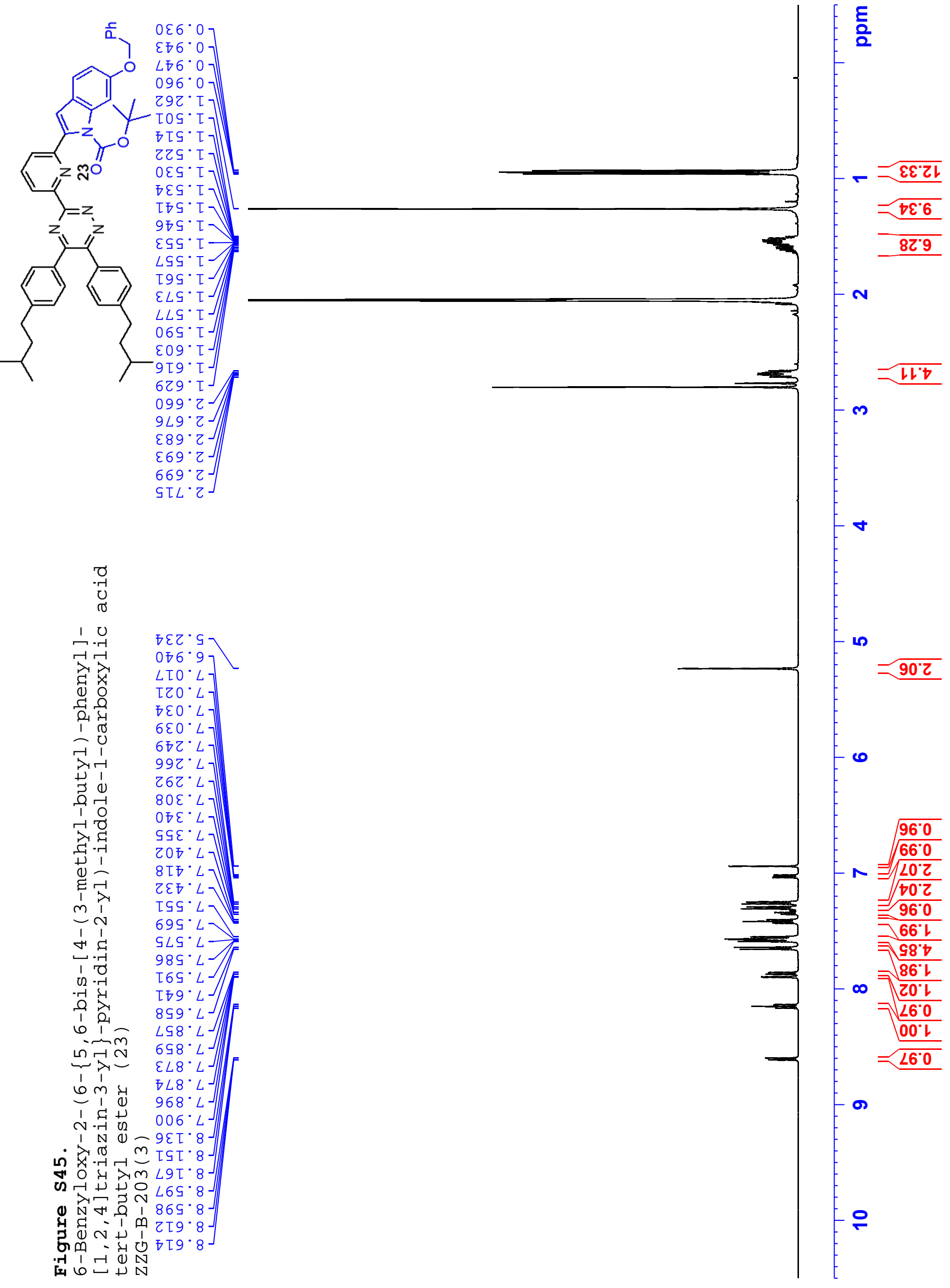




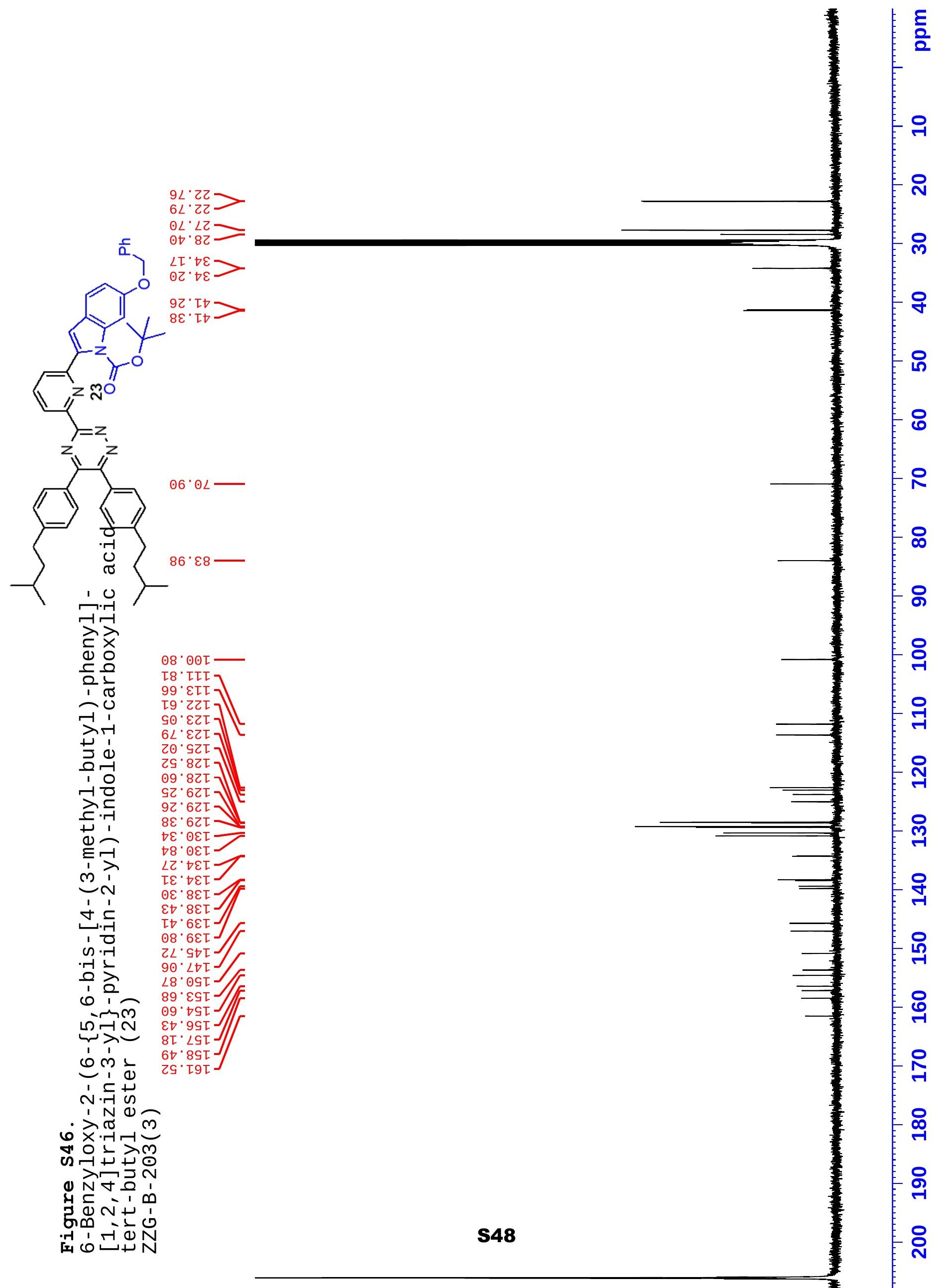




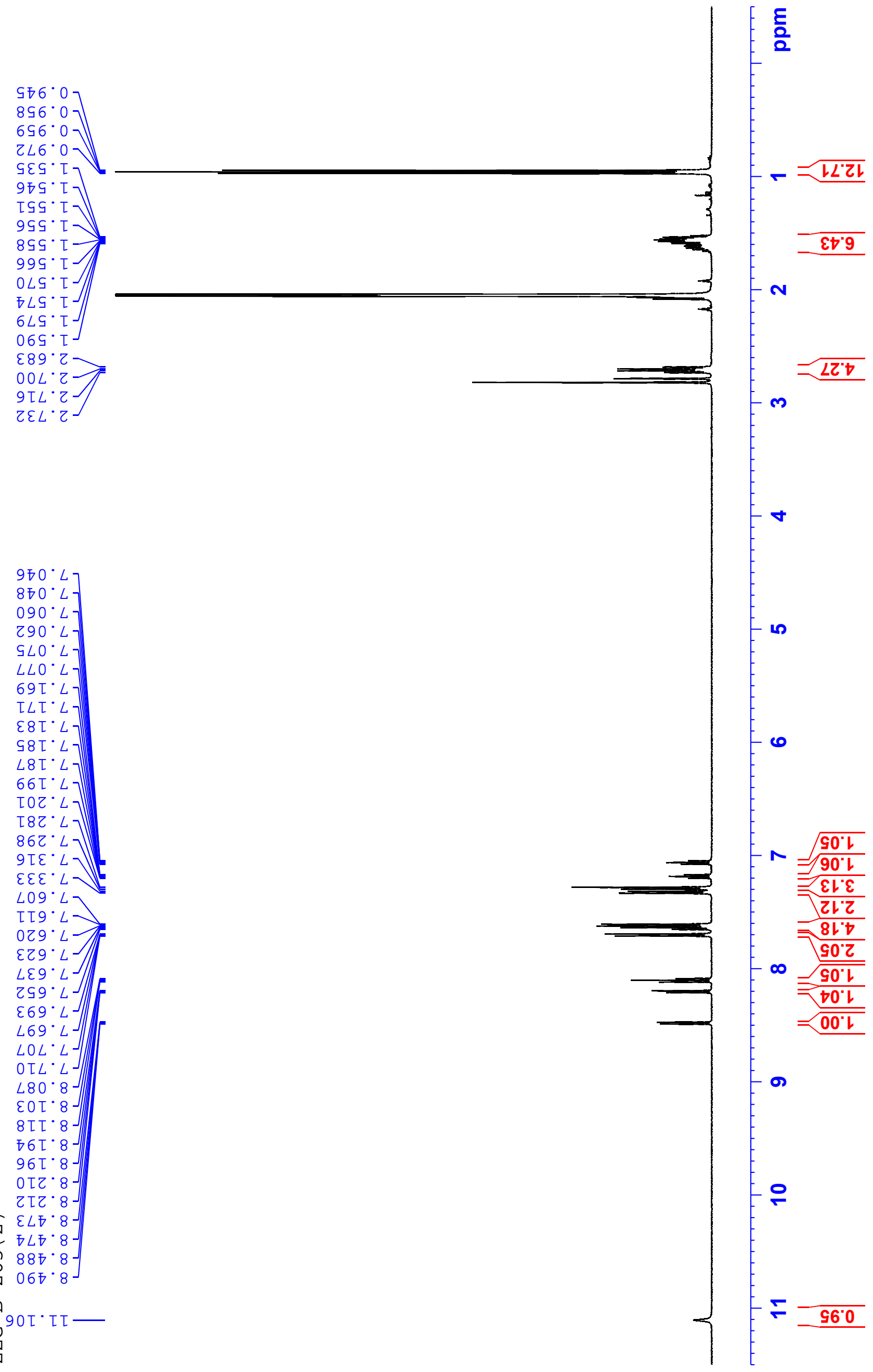




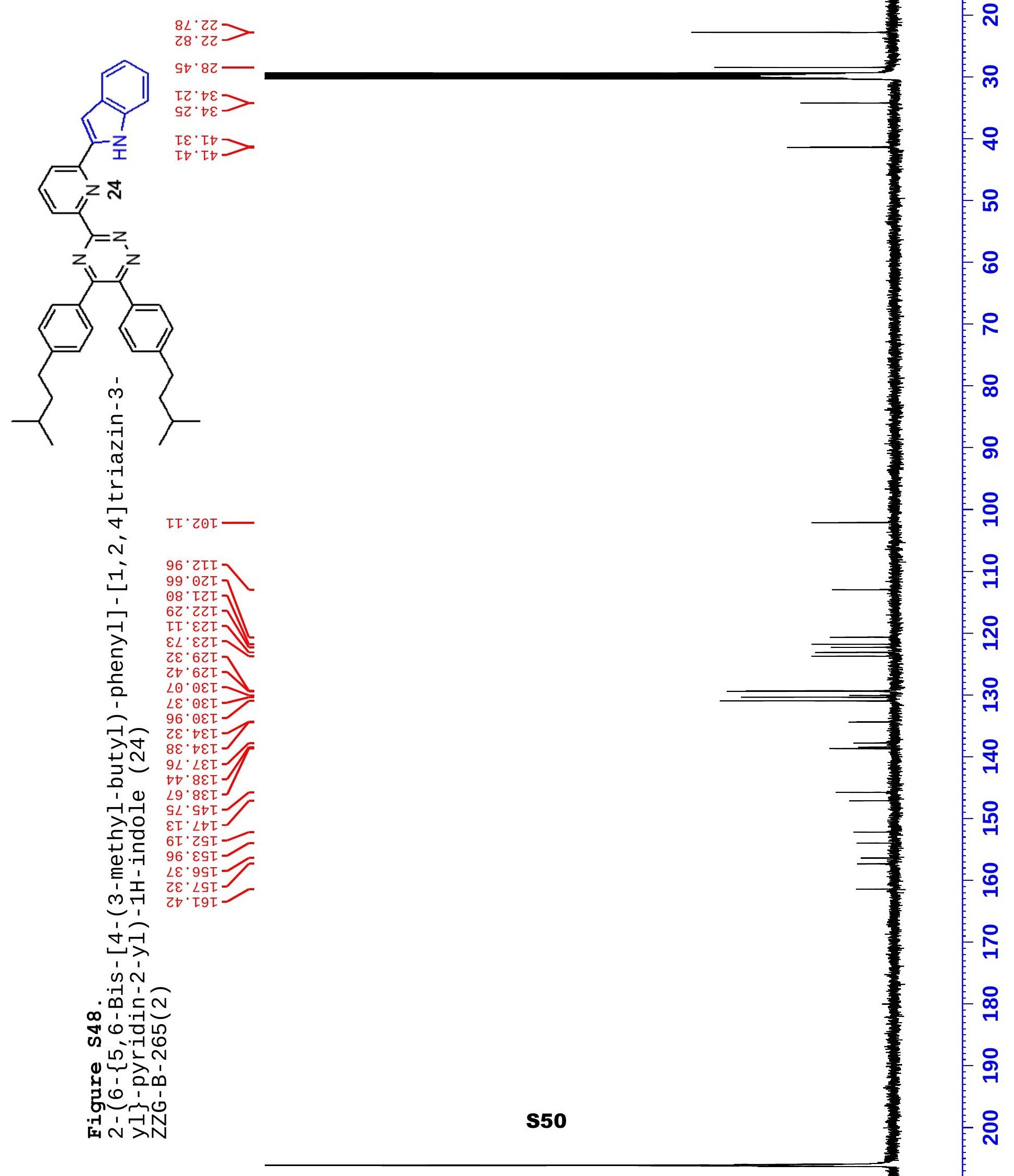




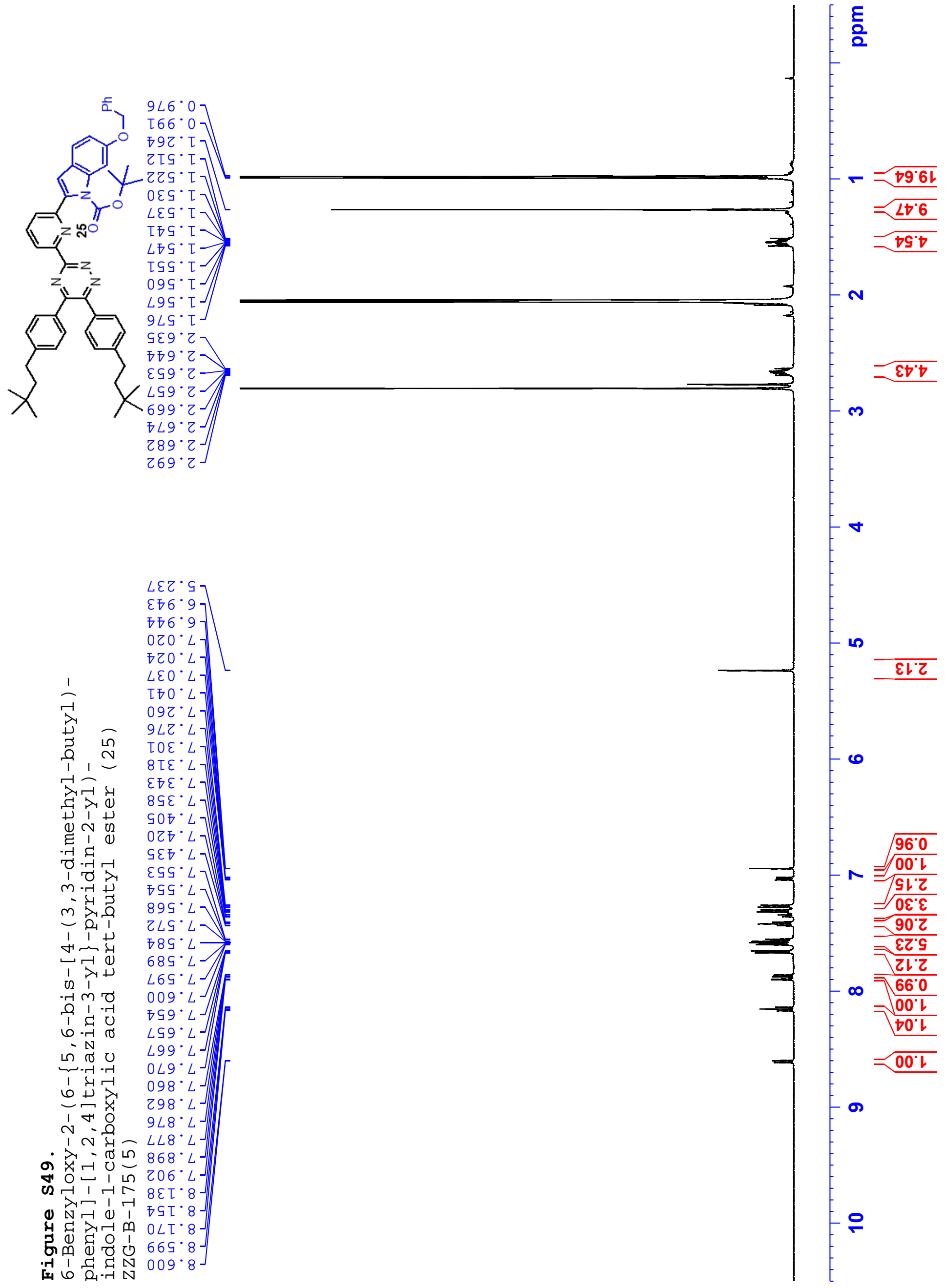




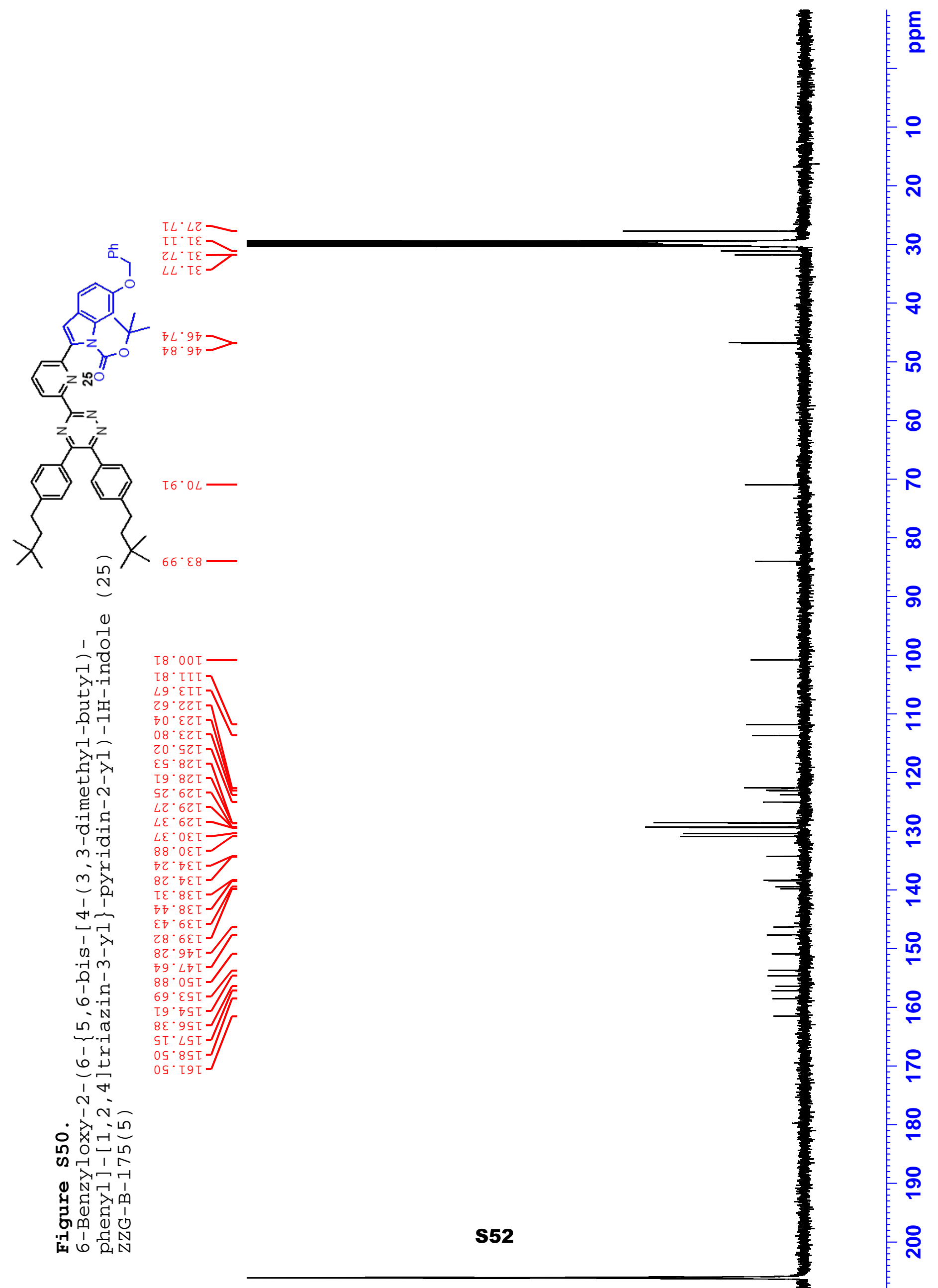



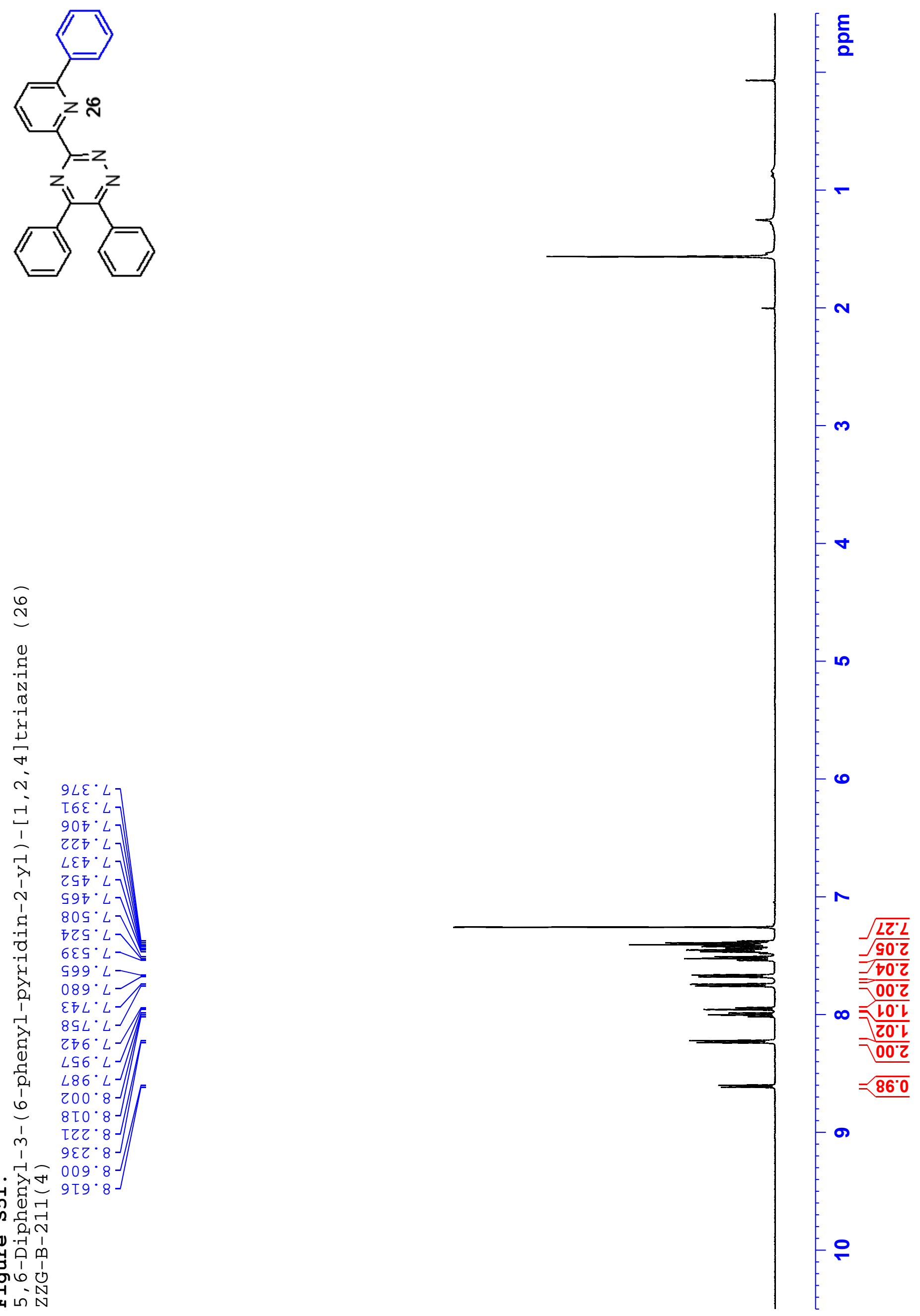


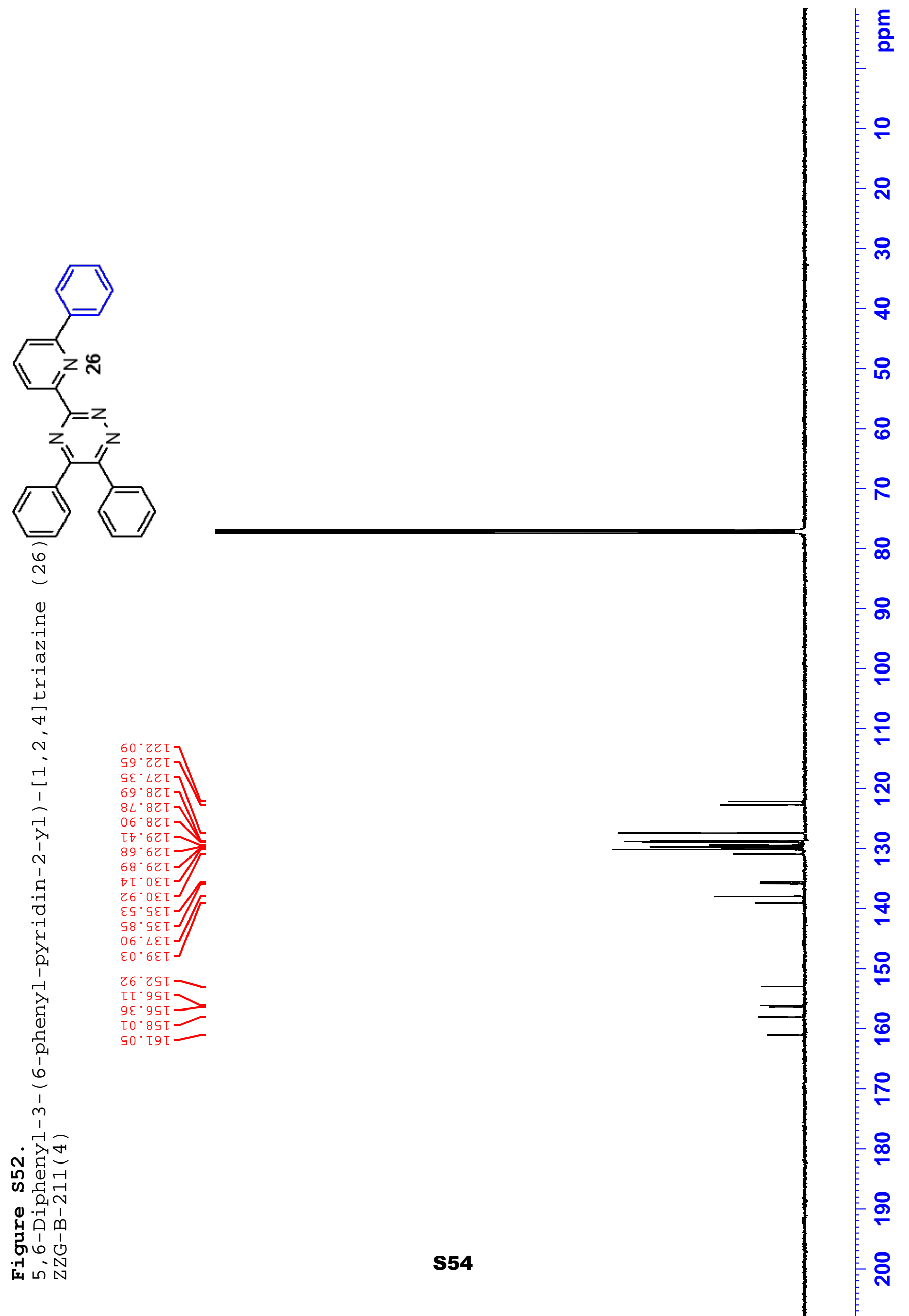


Figure S53. 1,2-bis-[4-(3-methyl-butyl)-phenyl]-ethane-1,2-dione Sample: ZZG-B-159

4x Column: 4

RediSep Column: Silica 24g

SN: E041508C98A253 Lot: 282217407X

Flow Rate: $35 \mathrm{ml} / \mathrm{min}$

Equilibration Volume: $168.0 \mathrm{ml}$

Initial Waste: $0.0 \mathrm{ml}$

Air Purge: 1.0 min

Solvent: A2 hexanes

Solvent: B2 (3:1) ethyl acetate:acetone
Peak Tube Volume: Max.

Non-Peak Tube Volume: Max.

Loading Type: Solid

Wavelength 1 (red): 254nm

Peak Width: 1 min

Threshold: 0.20 AU

Wavelength 2 (purple): 280nm

Run Notes:

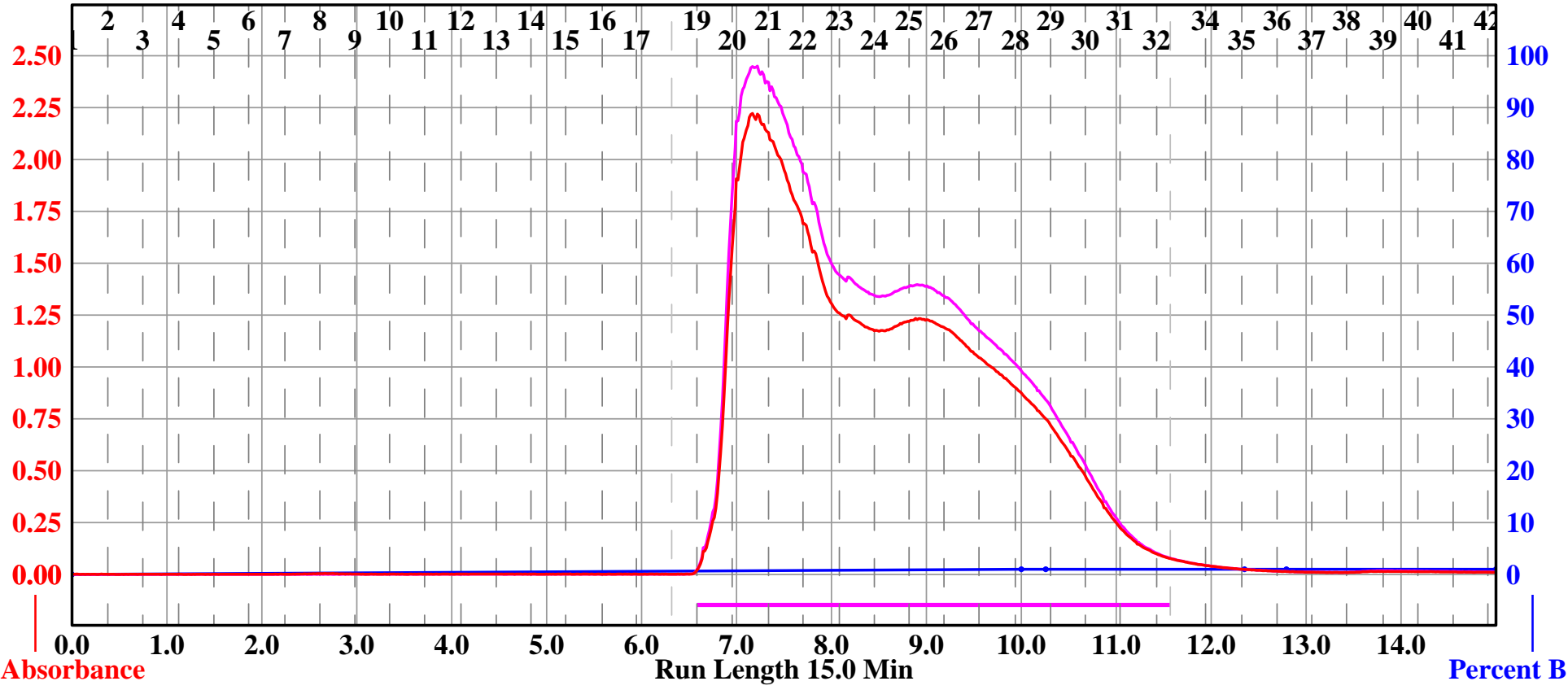

Rack A

\begin{tabular}{|c|c|c|c|}
\hline (1) & (72) & (73) & (74) \\
\hline (70) & (69) & (68) & (67) \\
\hline (61) & (62) & (63) & (64) \\
\hline (60) & (59) & (58) & (57) \\
\hline 51 & (52) & (33) & (54) \\
\hline 30 & (49) & (48) & (47) \\
\hline & (42) & (43) & (44) \\
\hline & (39) & (38) & (37) \\
\hline & (32) & (33) & (34) \\
\hline & (29) & (28) & (27) \\
\hline & (22) & (23) & (24) \\
\hline & (19) & (18) & (17) \\
\hline & (12) & (13) & (14) \\
\hline & (9) & (8) & (7) \\
\hline & (2) & (3) & (4) \\
\hline
\end{tabular}

$16 \mathrm{~mm} \times 100 \mathrm{~mm}$ Tubes

\begin{tabular}{|c|c|c|c|}
\hline \multicolumn{2}{|c|}{ Peak \# } & Start Tube & End Tube \\
\hline \multicolumn{2}{|c|}{1} & A:19 & \multirow[t]{2}{*}{ A:32 } \\
\hline Duration & $\% \mathrm{~B}$ & Solvent A & \\
\hline 0.0 & 0.0 & A2 dichlorometha & B2 methanol \\
\hline 10.0 & 1.0 & A2 dichlorometha & B2 methanol \\
\hline 0.3 & 1.0 & A2 dichlorometha & B2 methanol \\
\hline 2.1 & 1.0 & A2 dichlorometha & B2 methanol \\
\hline 0.4 & 1.0 & A2 dichlorometha & B2 methanol \\
\hline 2.2 & 1.0 & A2 dichlorometha & B2 methanol \\
\hline & & & \\
\hline & & & \\
\hline & & & \\
\hline
\end{tabular}


Figure S54. 3-(6-Bromo-pyridin-2-yl)-5,6-bis-[4-(3-methyl-butyl)-phenyl]-[1,2,4]triazine

Sample: ZZG-B-199

4x Column: 3

RediSep Column: Silica 24g

SN: E041508C987ED9 Lot: 282217407X

Flow Rate: $35 \mathrm{ml} / \mathrm{min}$

Equilibration Volume: $168.0 \mathrm{ml}$

Initial Waste: $0.0 \mathrm{ml}$

Air Purge: 1.0 min

Solvent: A2 hexanes

Solvent: B1 (3:1) ethyl acetate:acetone
Peak Tube Volume: Max.

Non-Peak Tube Volume: Max.

Loading Type: Solid

Wavelength 1 (red): 254nm

Peak Width: 1 min

Threshold: 0.20 AU

Wavelength 2 (purple): 280nm

Run Notes:

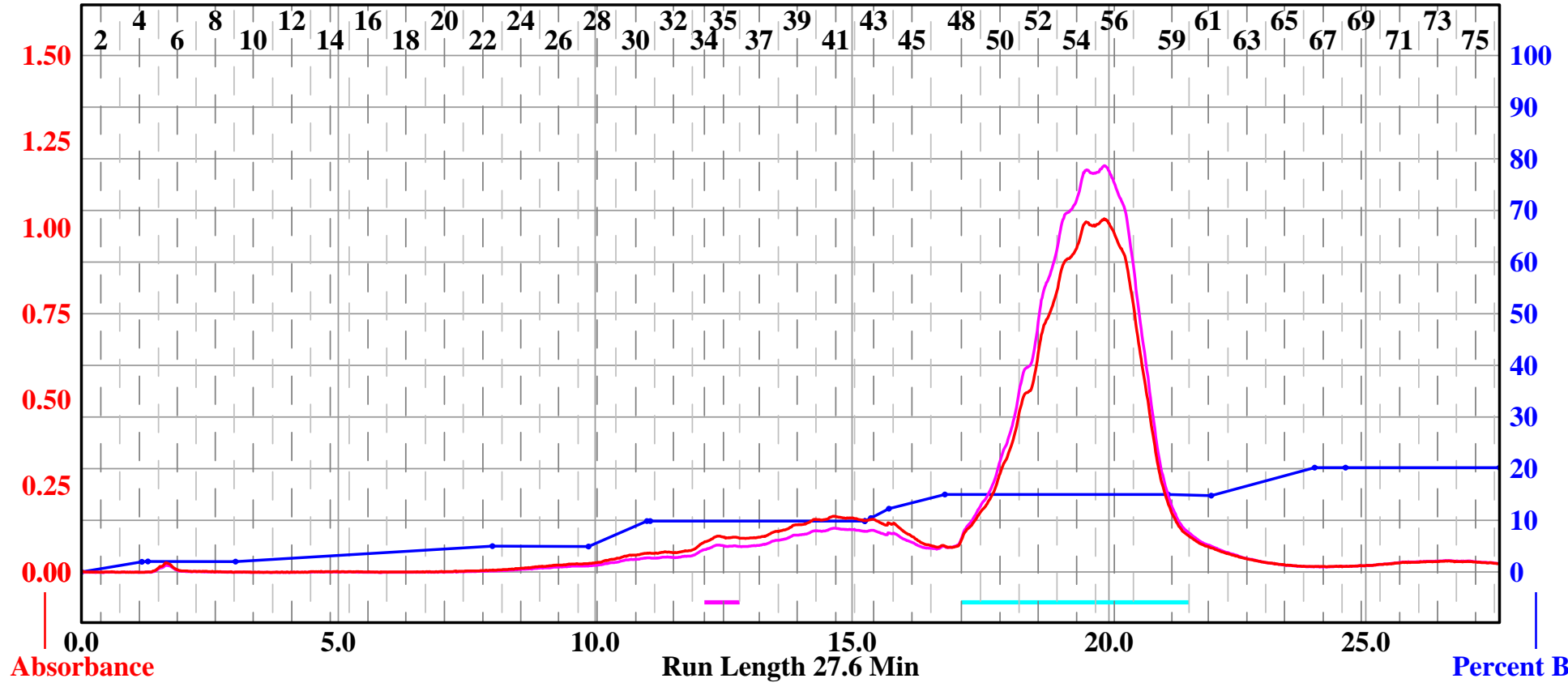

Rack A

Peak

\begin{tabular}{|c|c|c|c|}
\hline (71) & (72) & (73) & (74) \\
\hline (70) & (69) & (68) & (67) \\
\hline 61 & (62) & (63) & (64) \\
\hline (60) & (59) & (58) & (57) \\
\hline (e & (52) & (53) & (54) \\
\hline & (49) & (48) & (47) \\
\hline & (42) & (43) & (44) \\
\hline & (39) & (38) & (37) \\
\hline & (32) & (33) & (34) \\
\hline 3 & (29) & (28) & (27) \\
\hline & (22) & (23) & (24) \\
\hline & (19) & (18) & (17) \\
\hline & (12) & (13) & (14) \\
\hline & (9) & (8) & (7) \\
\hline & (2) & (3) & (4) \\
\hline
\end{tabular}

Start Tube

End Tube

\begin{tabular}{|c|c|c|c|}
\hline $\begin{array}{l}1 \\
2\end{array}$ & & $\begin{array}{l}A: 34 \\
A: 48\end{array}$ & $\begin{array}{l}A: 35 \\
A: 59\end{array}$ \\
\hline Duration & $\% \mathbf{B}$ & Solvent A & Solvent B \\
\hline 0.0 & 0.0 & A2 dichlorometha & B1 ethyl acetate \\
\hline 1.2 & 2.0 & A2 dichlorometha & B1 ethyl acetate \\
\hline 0.1 & 2.0 & A2 dichlorometha & B1 ethyl acetate \\
\hline 1.7 & 2.0 & A2 dichlorometha & B1 ethyl acetate \\
\hline 5.0 & 5.0 & A2 dichlorometha & B1 ethyl acetate \\
\hline 1.9 & 4.9 & A2 dichlorometha & B1 ethyl acetate \\
\hline 1.1 & 9.9 & A2 dichlorometha & B1 ethyl acetate \\
\hline 0.1 & 9.9 & A2 dichlorometha & B1 ethyl acetate \\
\hline 4.2 & 9.9 & A2 dichlorometha & B1 ethyl acetate \\
\hline $\begin{array}{l}0.0 \\
\ldots\end{array}$ & $\begin{array}{c}9.9 \\
\ldots\end{array}$ & $\begin{array}{l}\text { A2 dichlorometha } \\
. . .\end{array}$ & B1 ethyl acetate \\
\hline
\end{tabular}


Figure S55. 2-[6-(5,6-Diphenyl-[1,2,4]triazin-3-yl)-pyridin-2-yl]-5-methoxy-indole-1-carboxylic acid tert-butyl ester (2) Sample: ZZG-B-127

4x Column: 1

RediSep Column: Al2O3 pH=4 8g

Flow Rate: $18 \mathrm{ml} / \mathrm{min}$ Equilibration

Volume: $33.6 \mathrm{ml}$ Initial Waste: $0.0 \mathrm{ml}$

Air Purge: $1.5 \mathrm{~min}$

Solvent: A2 hexanes

Solvent: B2 (3:1) ethyl acetate:acetone
Peak Tube Volume: Max.

Non-Peak Tube Volume: Max.

Loading Type: Solid

Wavelength 1 (red): 254nm

Peak Width: $30 \mathrm{sec}$

Threshold: 0.20 AU

Wavelength 2 (purple): $280 \mathrm{~nm}$

Run Notes:

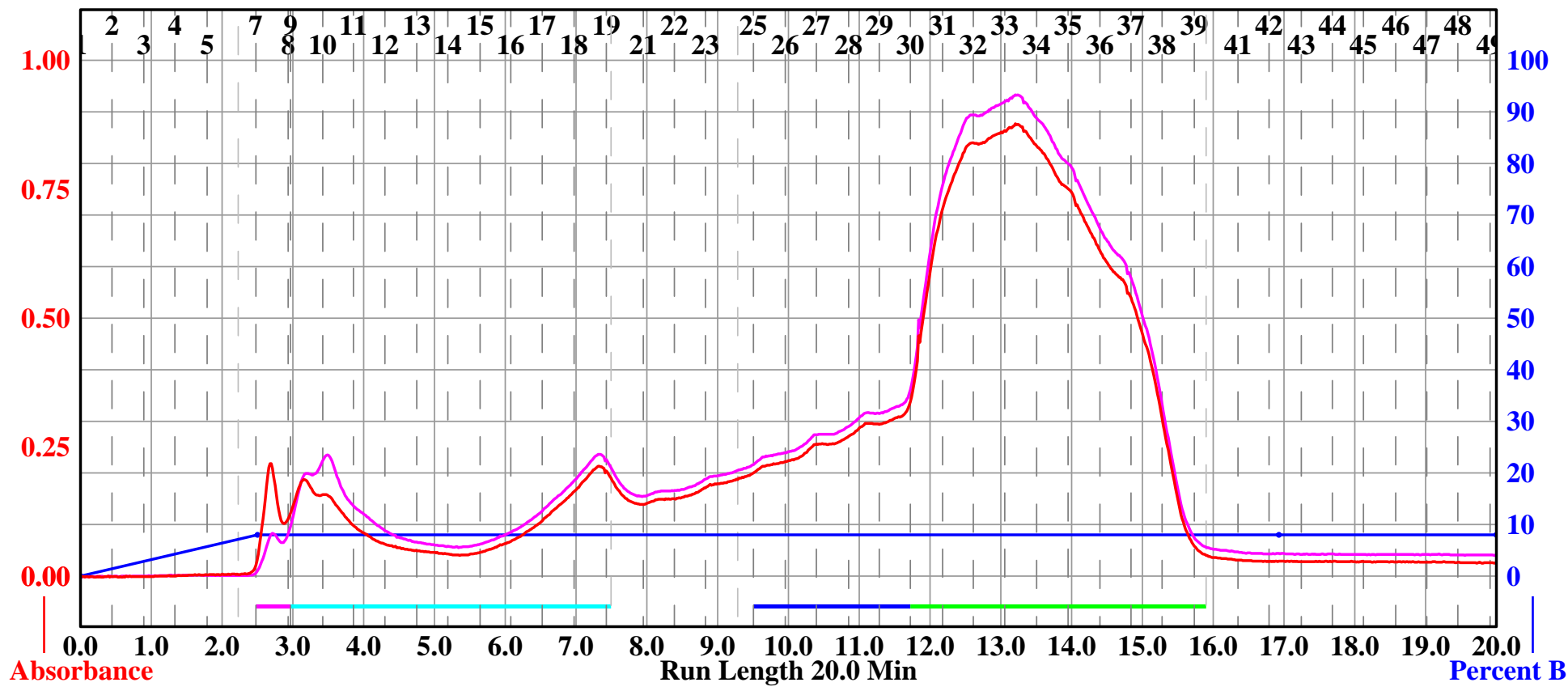

Rack A

Peak \#

Start Tube

End Tube

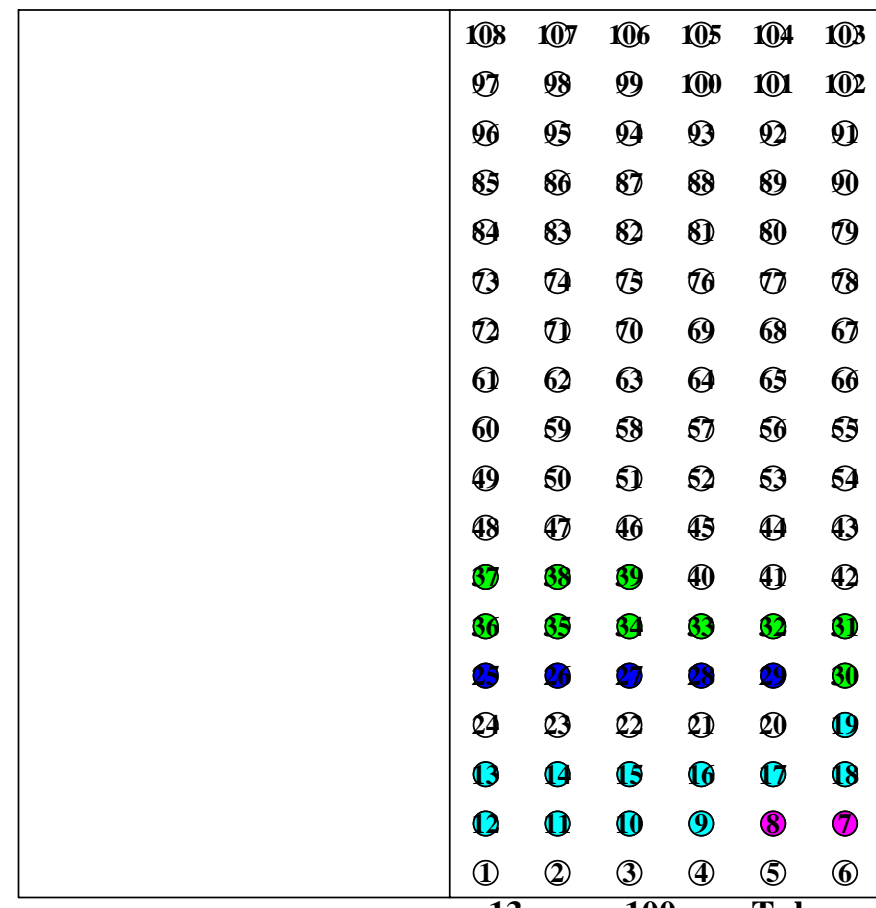

$13 \mathrm{~mm} \times 100 \mathrm{~mm}$ Tubes

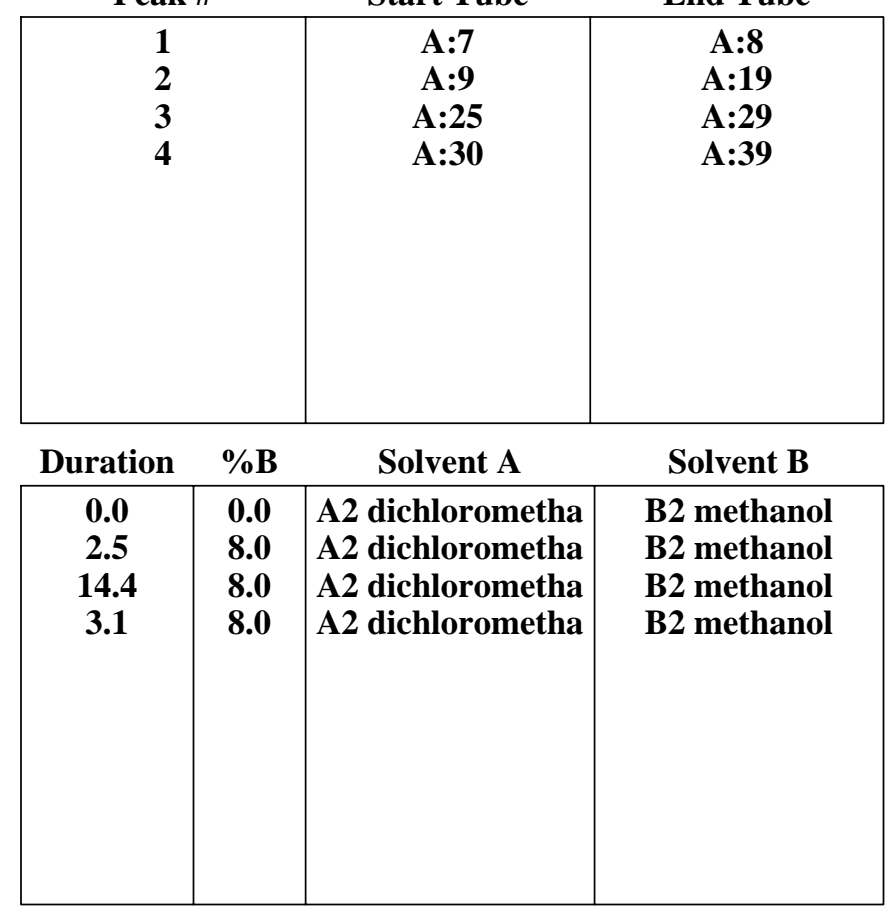


Figure S56. 2-[6-(5,6-Diphenyl-[1,2,4]triazin-3-yl)-pyridin-2-yl]-indole-1-carboxylic acid tert-butyl ester (3) Sample: MLT-A-103

4x Column: 1

RediSep Column: $\mathrm{Al2O3} \mathrm{pH}=48 \mathrm{~g}$

Flow Rate: $18 \mathrm{ml} / \mathrm{min}$ Equilibration

Volume: $33.6 \mathrm{ml}$ Initial Waste: $0.0 \mathrm{ml}$

Air Purge: $1.5 \mathrm{~min}$

Solvent: A2 hexanes

Solvent: B2 (3:1) ethyl acetate:acetone
Peak Tube Volume: $8 \mathrm{ml}$

Non-Peak Tube Volume: $8 \mathrm{ml}$

Loading Type: Solid

Wavelength 1 (red): 254nm

Peak Width: 30 sec

Threshold: 0.20 AU

Wavelength 2 (purple): 280nm

Run Notes:

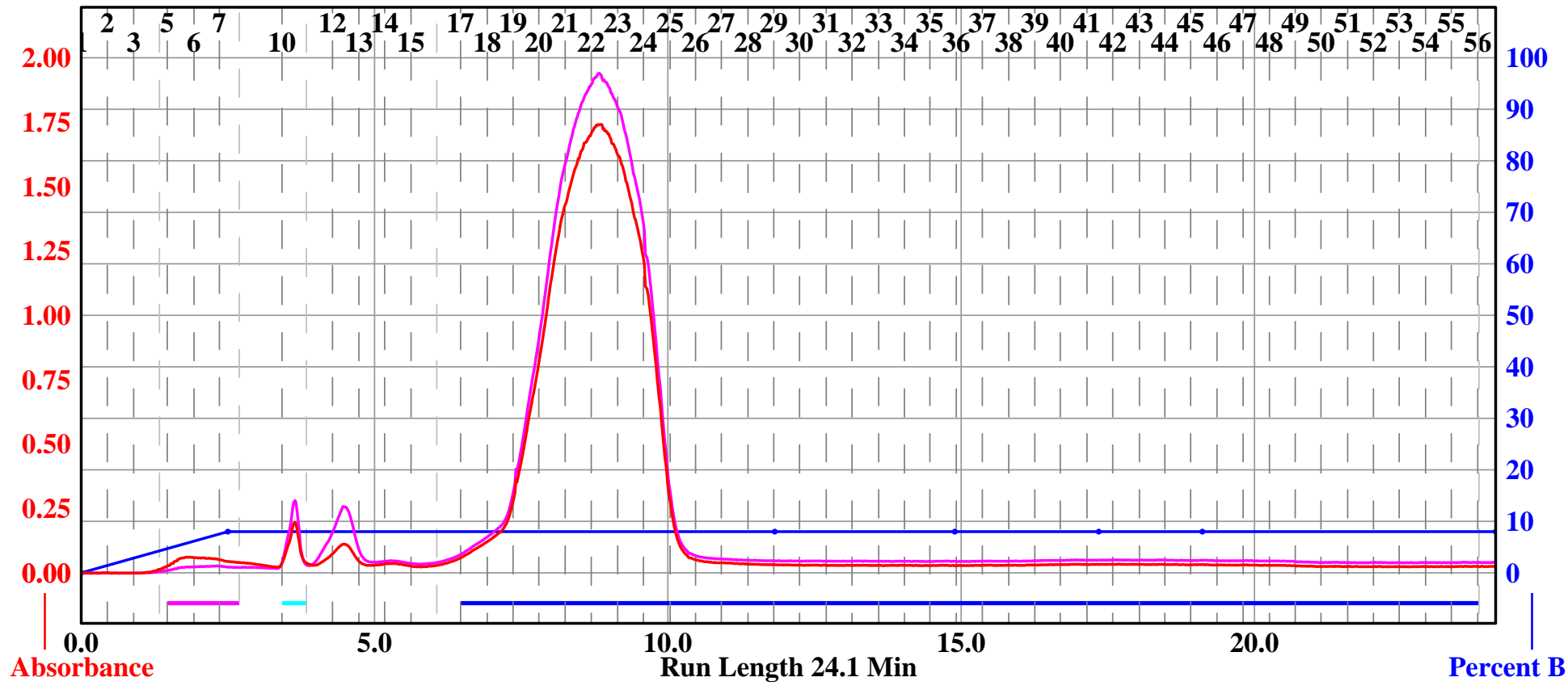

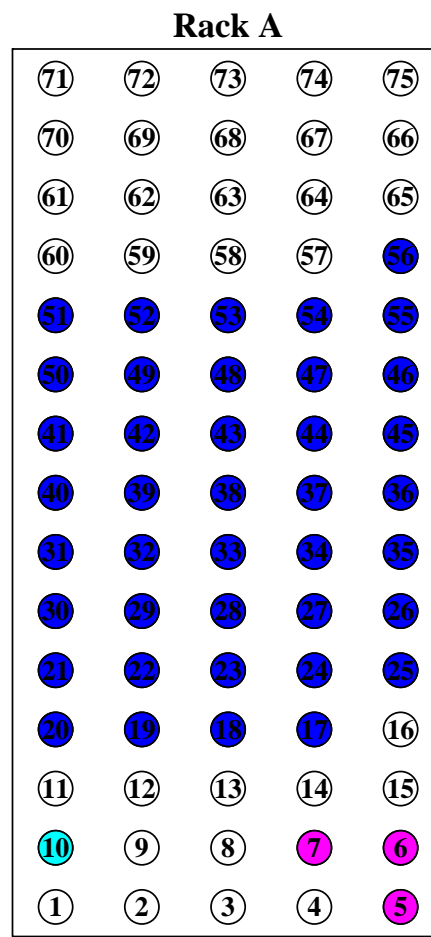

$16 \mathrm{~mm} \times 100 \mathrm{~mm}$ Tubes

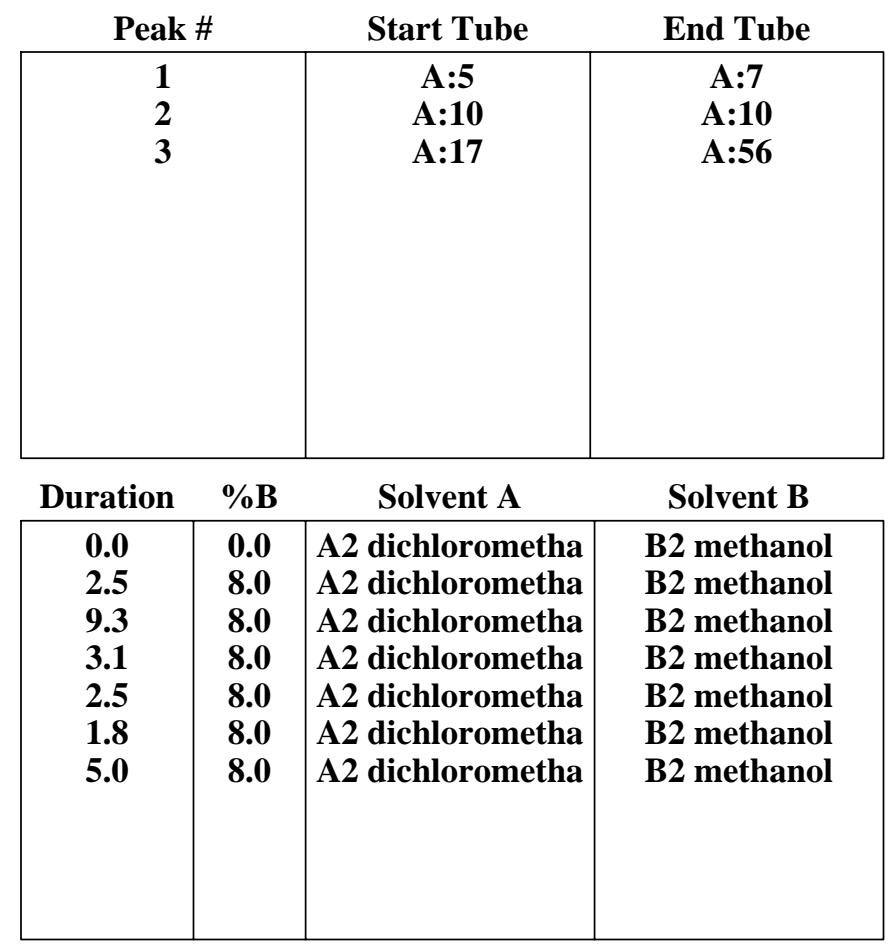


Figure S57. 2-[6-(5,6-Diphenyl-[1,2,4]triazin-3-yl)-pyridin-2-yl]-4-methyl-indole-1-carboxylic acid tert-butyl ester (4) Sample: ZZG-B-135

4x Column: 1

RediSep Column: Al2O3 pH=4 8g

Flow Rate: $18 \mathrm{ml} / \mathrm{min}$

Equilibration Volume: $33.6 \mathrm{ml}$

Initial Waste: $0.0 \mathrm{ml}$

Air Purge: 1.5 min

Solvent: A2 hexanes

Solvent: B2 (3:1) ethyl

acetate:acetone
Peak Tube Volume: $8 \mathrm{ml}$

Non-Peak Tube Volume: $8 \mathrm{ml}$

Loading Type: Solid

Wavelength 1 (red): 254nm

Peak Width: 30 sec

Threshold: 0.20 AU

Wavelength 2 (purple): 280nm

Run Notes:

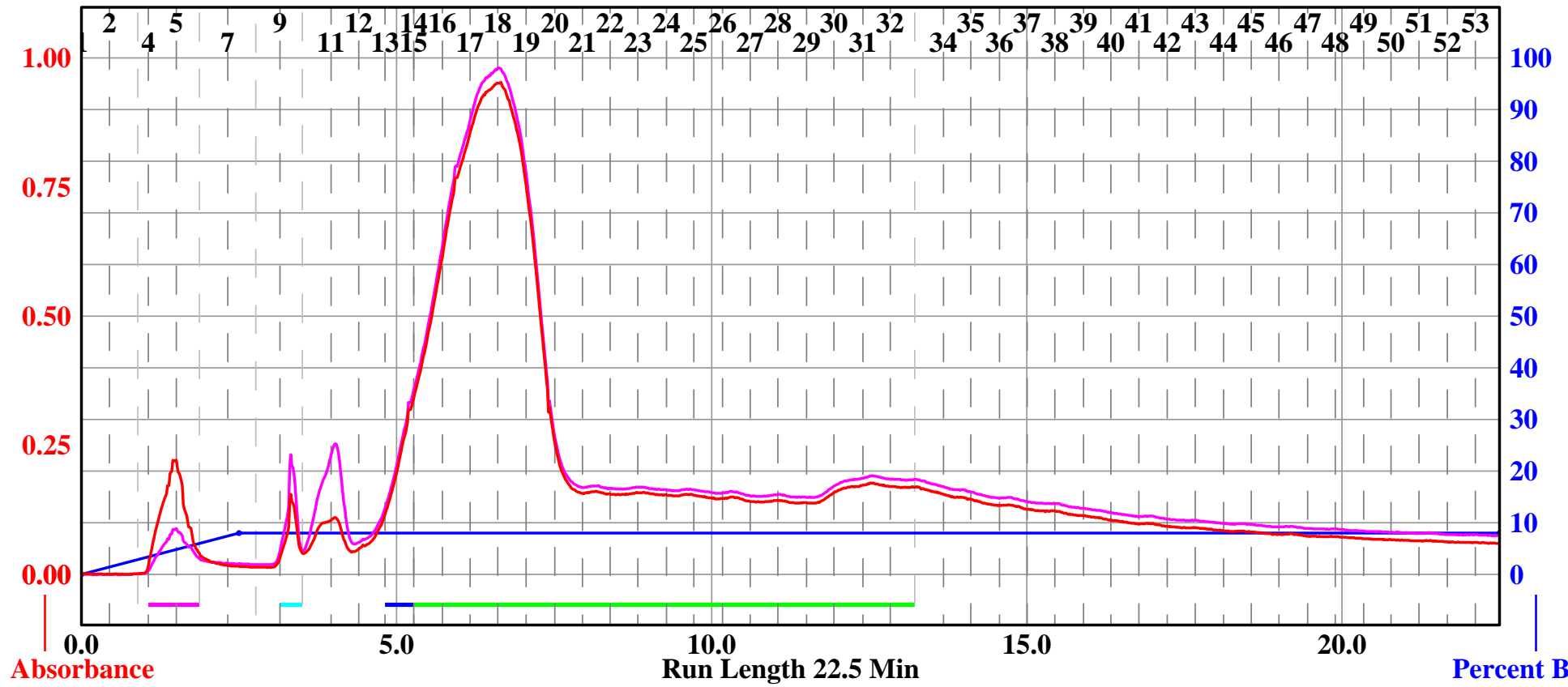

\begin{tabular}{|c|c|c|c|c|c|}
\hline \multicolumn{6}{|c|}{ Rack $A$} \\
\hline (๑) & (107 & 106 & 105 & (104 & \\
\hline 27 & (g) & (9) & 100 & (101 & (1) \\
\hline fo & (2) & $\mathscr{\theta}$ & (3) & (2) & Q1 \\
\hline 53 & 80 & 87 & 88 & 89 & 20 \\
\hline 84 & 83 & 82 & (D) & (10) & (19) \\
\hline 3 & 89 & (18) & (20) & $(77$ & 8 \\
\hline 12 & (71) & (11) & 69 & 68 & 6 \\
\hline (1) & (2) & 6 & (4) & 68 & 6 \\
\hline (1) & (9) & 58 & 57 & ש & 5 \\
\hline (49) & (D) & (1) & 52 & 53 & 5 \\
\hline 48 & (47) & (40) & 45 & (4) & 43 \\
\hline (37) & Bg & B9 & (10) & (41) & (4) \\
\hline (b) & B5 & B & B3 & (32) & 3 \\
\hline 25 & (2) & (2) & 8 & (2) & (3) \\
\hline & 23 & (2) & (2) & (2) & \\
\hline & 0 & (1) & (6) & (1) & ( \\
\hline (12) & (11) & (10) & (D) & (8) & (7) \\
\hline (1) & (2) & (3) & (4) & (5) & \\
\hline
\end{tabular}

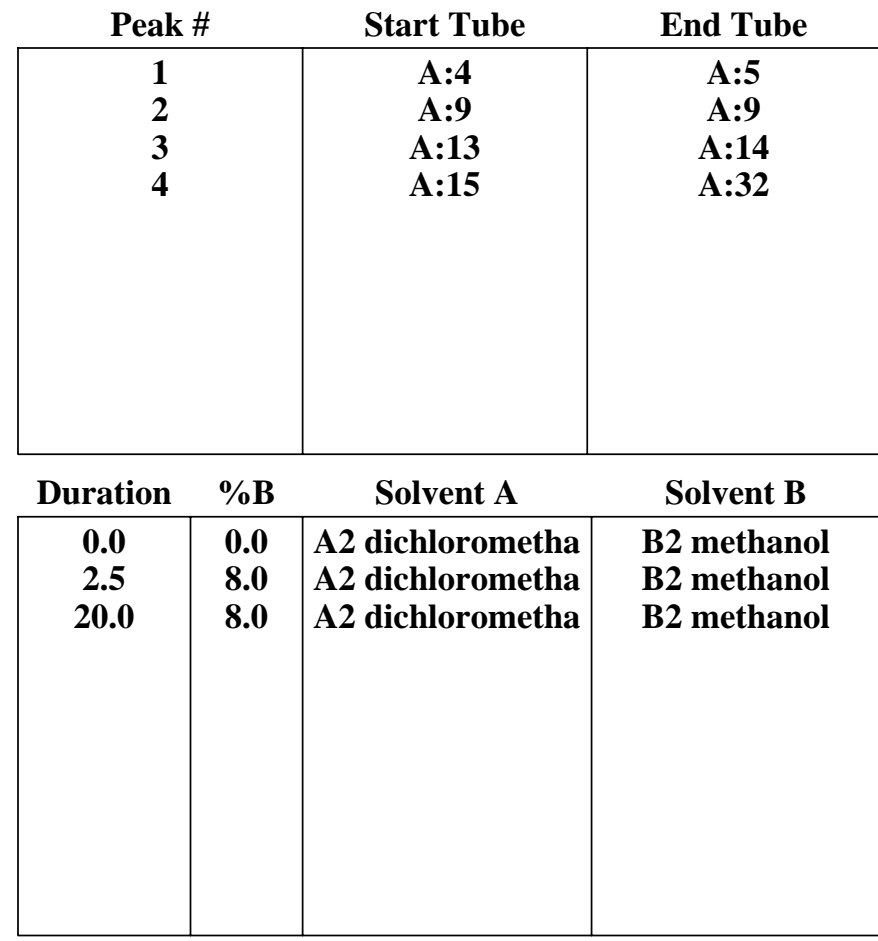

$13 \mathrm{~mm} \times 100 \mathrm{~mm}$ Tubes 
Figure S58. 2-[6-(5,6-Diphenyl-[1,2,4]triazin-3-yl)-pyridin-2-yl]-5-fluoro-indole-1-carboxylic acid tert-butyl ester (5) Sample: MLT-A-175

4x Column: 4

RediSep Column: Al2O3 pH=4 8g

Flow Rate: $18 \mathrm{ml} / \mathrm{min}$

Equilibration Volume: $33.6 \mathrm{ml}$

Initial Waste: $0.0 \mathrm{ml}$

Air Purge: 0.5 min

Solvent: A2 hexanes

Solvent: B2 (3:1) ethyl

acetate:acetone
Peak Tube Volume: Max.

Non-Peak Tube Volume: Max.

Loading Type: Solid

Wavelength 1 (red): 254nm

Peak Width: 30 sec

Threshold: 0.20 AU

Wavelength 2 (purple): 280nm

Run Notes:

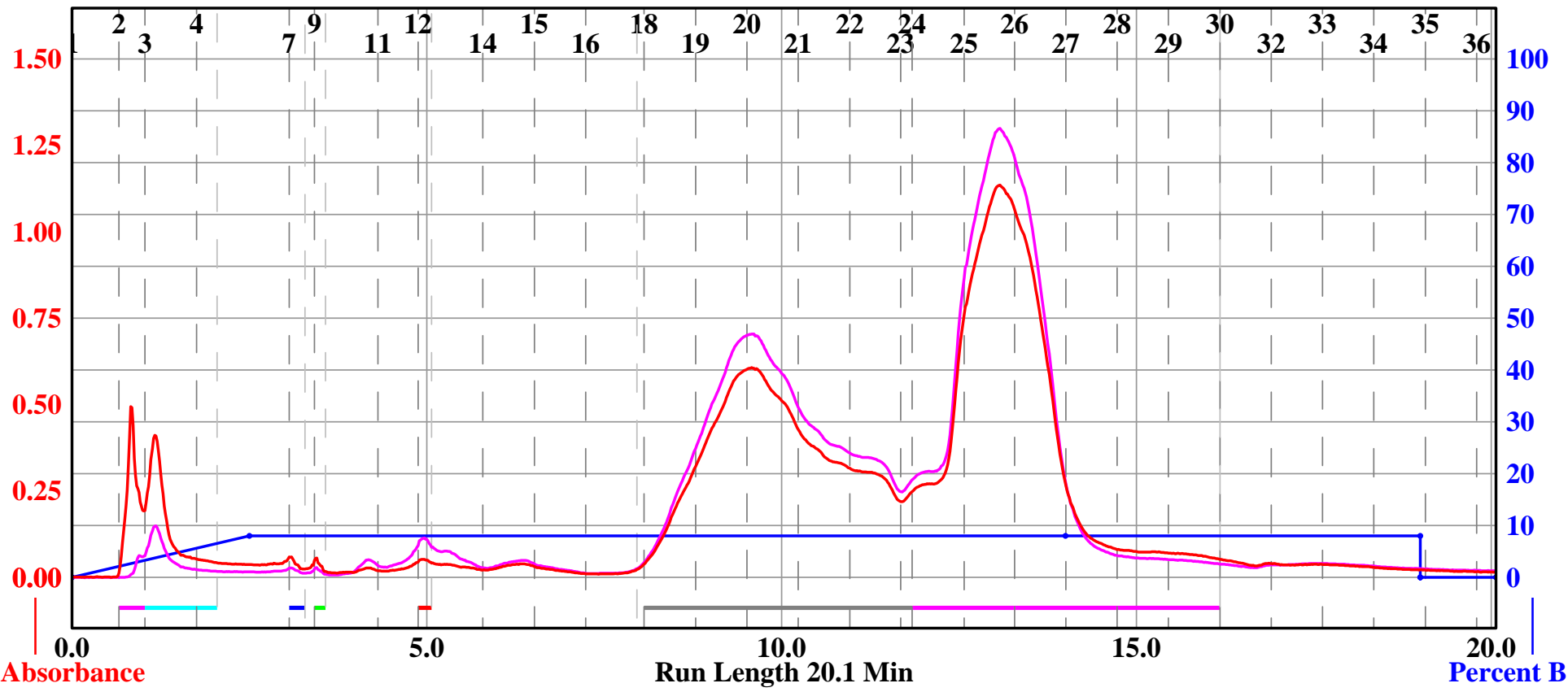

Rack A

\begin{tabular}{|c|c|c|c|}
\hline (71) & (72) & (73) & (74) \\
\hline (70) & (69) & (68) & (67) \\
\hline 61 & (62) & (63) & (64) \\
\hline (60) & (99) & (58) & (57) \\
\hline (51) & (52) & (33) & (54) \\
\hline (50) & (49) & (48) & (47) \\
\hline (41) & (42) & (43) & (44) \\
\hline (40) & (39) & (38) & (37) \\
\hline (31) & (32) & (33) & (34) \\
\hline (30) & (29) & (28) & (27) \\
\hline (21) & (22) & (23) & (24) \\
\hline (20) & (19) & (18) & (17) \\
\hline (11) & (12) & (13) & (14) \\
\hline (10) & (9) & (8) & (7) \\
\hline (1) & (2) & (3) & (4) \\
\hline
\end{tabular}

Start Tube

A:2

A:3

A:7

A:9

A:12

A:18

A:24

A:23

A:30

A:4

A:7

A:9

A:12

7

\begin{tabular}{|c|c|c|c|}
\multicolumn{1}{c}{ Duration } & \multicolumn{1}{c}{ \% B } & \multicolumn{1}{c}{ Solvent A } & Solvent B \\
\hline $\mathbf{0 . 0}$ & $\mathbf{0 . 0}$ & A2 dichlorometha & B2 methanol \\
$\mathbf{2 . 5}$ & $\mathbf{8 . 0}$ & A2 dichlorometha & B2 methanol \\
$\mathbf{1 1 . 5}$ & $\mathbf{8 . 0}$ & A2 dichlorometha & B2 methanol \\
$\mathbf{5 . 0}$ & $\mathbf{8 . 0}$ & A2 dichlorometha & B2 methanol \\
$\mathbf{0 . 0}$ & $\mathbf{0 . 0}$ & A2 dichlorometha & B2 methanol \\
$\mathbf{0 . 0}$ & $\mathbf{0 . 0}$ & A2 dichlorometha & B2 methanol \\
$\mathbf{1 . 1}$ & $\mathbf{0 . 0}$ & A2 dichlorometha & B2 methanol \\
& & & \\
& & & \\
& & & \\
\hline
\end{tabular}


Figure S59. 2-[6-(5,6-Diphenyl-[1,2,4]triazin-3-yl)-pyridin-2-yl]-5-bromo-indole-1-carboxylic acid tert-butyl ester (6) Sample: ZZG-B-133

4x Column: 1

RediSep Column: Al2O3 pH=4 $8 \mathrm{~g}$

Flow Rate: $18 \mathrm{ml} / \mathrm{min}$

Equilibration Volume: $33.6 \mathrm{ml}$

Initial Waste: $0.0 \mathrm{ml}$

Air Purge: $1.5 \mathrm{~min}$

Solvent: A2 hexanes

Solvent: B2 (3:1) ethyl

acetate:acetone
Peak Tube Volume: $8 \mathrm{ml}$

Non-Peak Tube Volume: $8 \mathrm{ml}$

Loading Type: Solid

Wavelength 1 (red): 254nm

Peak Width: 30 sec

Threshold: 0.20 AU

Wavelength 2 (purple): 280nm

Run Notes:

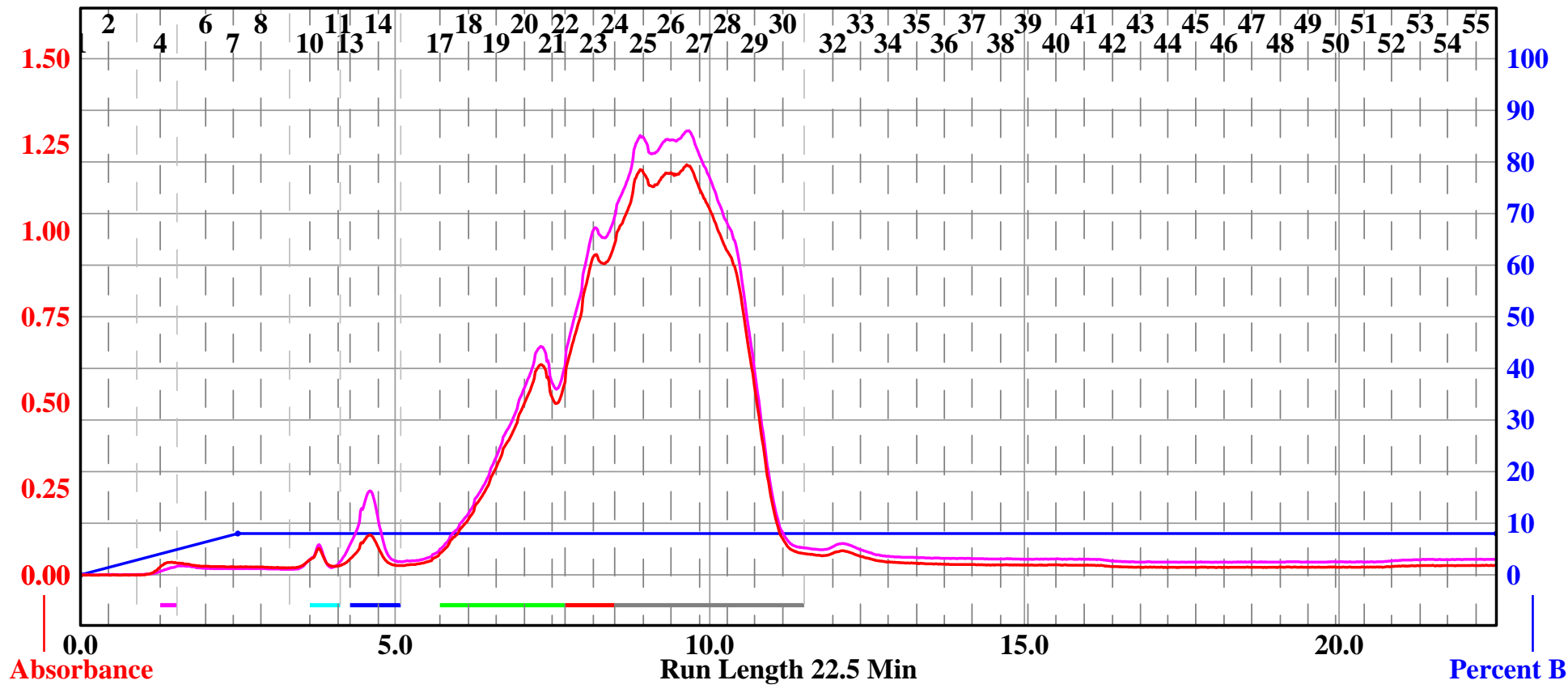

\begin{tabular}{|c|c|c|c|c|c|}
\hline \multicolumn{6}{|c|}{ Rack A } \\
\hline 108 & (107) & 106 & 105 & (104 & \\
\hline 27 & $\mathscr{Q}$ & (29) & 100 & (101 & \\
\hline d) & (2) & (9) & (3) & (2) & \\
\hline 35 & 8 & 87 & 88 & 89 & \\
\hline 84 & 8 & 82 & $\$$ & 80 & \\
\hline 13 & 87 & 83 & (ש6) & (27) & \\
\hline (12) & (11) & (11) & (9) & 68 & \\
\hline (1) & (2) & (3) & (3) & (6) & \\
\hline (10 & 59 & 58 & 57 & b & \\
\hline (49) & (10) & (D) & 22 & 53 & \\
\hline 48 & 48 & (46) & (45) & (4) & \\
\hline B77 & B8 & (39) & (40) & (41) & \\
\hline b & WG & B9 & B3 & (32) & \\
\hline 25 & 2 & (2) & 8 & 9 & \\
\hline (2) & (2) & 2 & (2) & (21) & \\
\hline 0 & 0 & (15) & (16) & (17) & \\
\hline (12) & (1) & (11) & (D) & (8) & \\
\hline (1) & (2) & (3) & (4) & (5) & \\
\hline
\end{tabular}

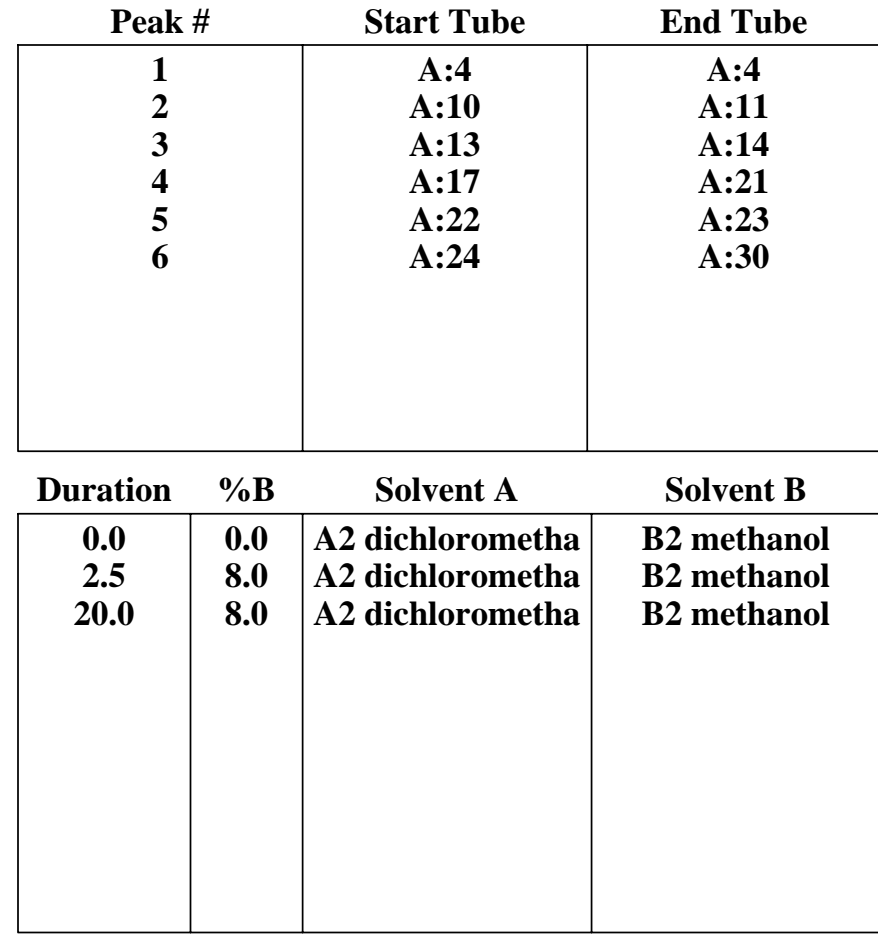

$13 \mathrm{~mm} \times 100 \mathrm{~mm}$ Tubes 
Figure S60. 5-(tert-Butyl-dimethyl-silanyloxy)-2-[6-(5,6-diphenyl-[1,2,4]triazin-3-yl)-pyridin-2-yl]-indole-1-carboxylic acid tert-butyl ester (7)

Sample: ZZG-B-161

Rf 200

Monday 25 February 2019 04:40PM

4x Column: 1

RediSep Column: Al2O3 pH=4 8g

Flow Rate: $18 \mathrm{ml} / \mathrm{min}$

Equilibration Volume: $33.6 \mathrm{ml}$

Initial Waste: $0.0 \mathrm{ml}$

Air Purge: 1.5 min

Solvent: A2 hexanes

Solvent: B2 3:1 ethyl

Peak Tube Volume: $8 \mathrm{ml}$

Non-Peak Tube Volume: $8 \mathrm{ml}$

Loading Type: Solid

Wavelength 1 (red): 254nm

Peak Width: 30 sec

Threshold: 0.20 AU

Wavelength 2 (purple): 280nm

acetate:acetone

Run Notes:

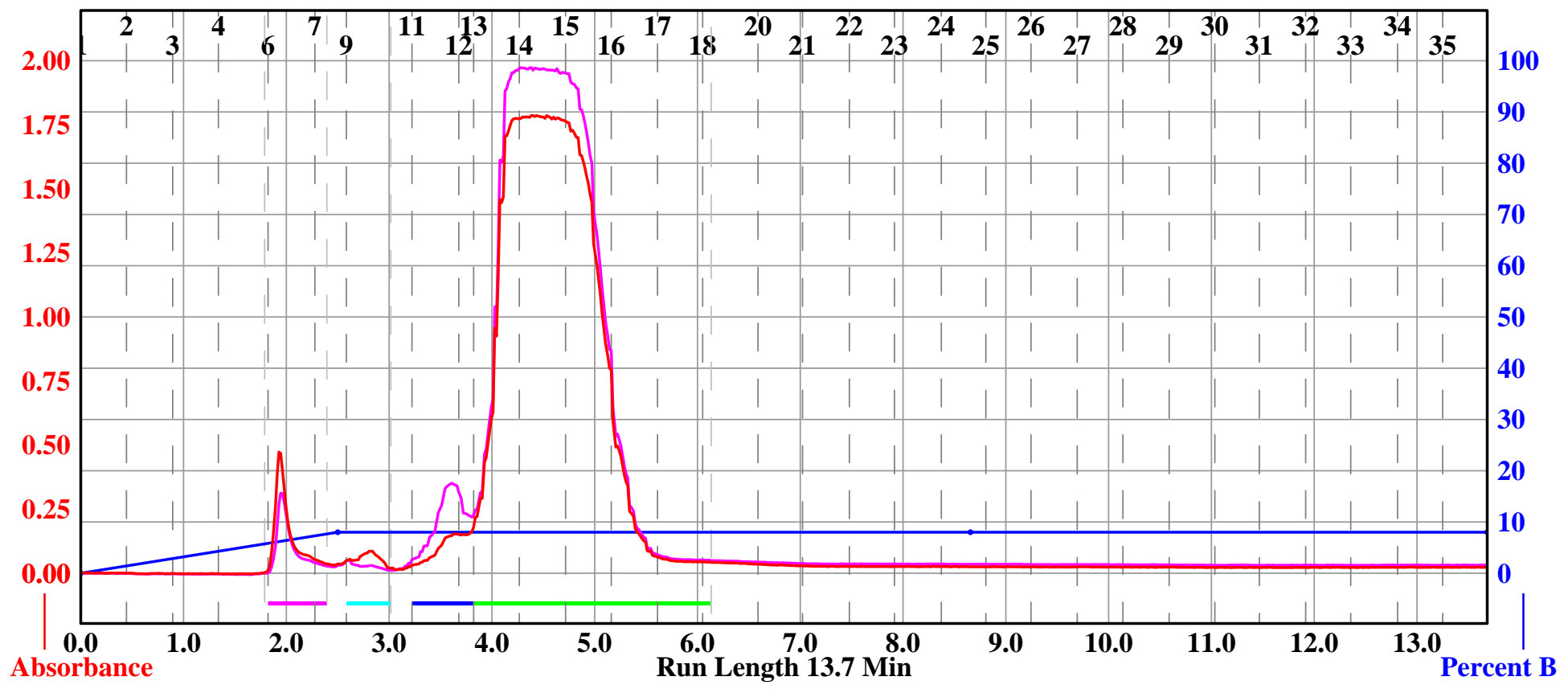

Absorbance

Rack A

Peak \#

Start Tube

End Tube

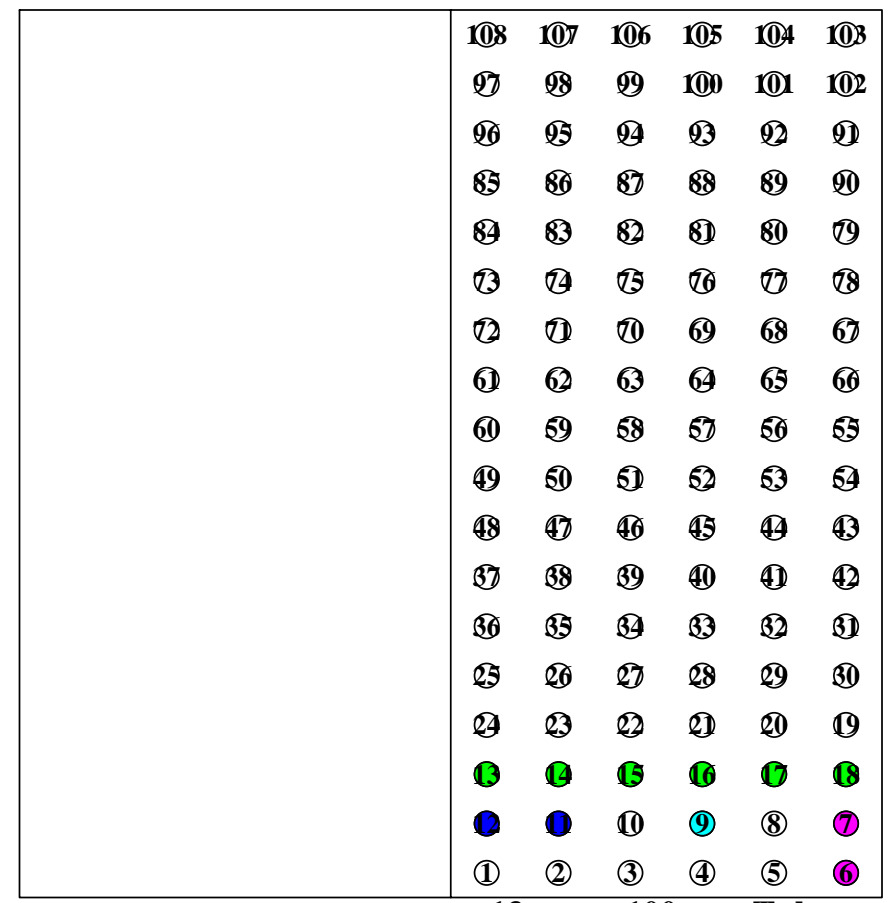

$13 \mathrm{~mm} \times 100 \mathrm{~mm}$ Tubes

\begin{tabular}{|c|c|c|c|}
\hline $\begin{array}{l}\mathbf{1} \\
\mathbf{2} \\
\mathbf{3} \\
\mathbf{4}\end{array}$ & & $\begin{array}{c}\text { A:6 } \\
\text { A:9 } \\
\text { A:11 } \\
\text { A:13 }\end{array}$ & $\begin{array}{c}\text { A:7 } \\
\text { A:9 } \\
\text { A:12 } \\
\text { A:18 }\end{array}$ \\
\hline Duration & $\%$ B & Solvent A & Solvent B \\
\hline $\begin{array}{l}0.0 \\
2.5 \\
6.2 \\
5.0\end{array}$ & $\begin{array}{l}\text { 0.0 } \\
8.0 \\
8.0 \\
8.0\end{array}$ & $\begin{array}{l}\text { A2 dichlorometha } \\
\text { A2 dichlorometha } \\
\text { A2 dichlorometha } \\
\text { A2 dichlorometha }\end{array}$ & $\begin{array}{l}\text { B2 methanol } \\
\text { B2 methanol } \\
\text { B2 methanol } \\
\text { B2 methanol }\end{array}$ \\
\hline
\end{tabular}


Figure S61. 6-Benzyloxy-2-[6-(5,6-diphenyl-[1,2,4]triazin-3-yl)-pyridin-2-yl]-indole-1-carboxylic acid tert-butyl ester (8) Sample: ZZG-B-145

4x Column: 1

RediSep Column: Al2O3 pH=4 8g

Flow Rate: $18 \mathrm{ml} / \mathrm{min}$

Equilibration Volume: $33.6 \mathrm{ml}$

Initial Waste: $0.0 \mathrm{ml}$

Air Purge: 1.5 min

Solvent: A2 hexanes

Solvent: B2 (3:1) ethyl

acetate:acetone
Peak Tube Volume: $8 \mathrm{ml}$

Non-Peak Tube Volume: $8 \mathrm{ml}$

Loading Type: Solid (Pause)

Wavelength 1 (red): 254nm

Peak Width: 30 sec

Threshold: $0.20 \mathrm{AU}$

Wavelength 2 (purple): 280nm

Run Notes:

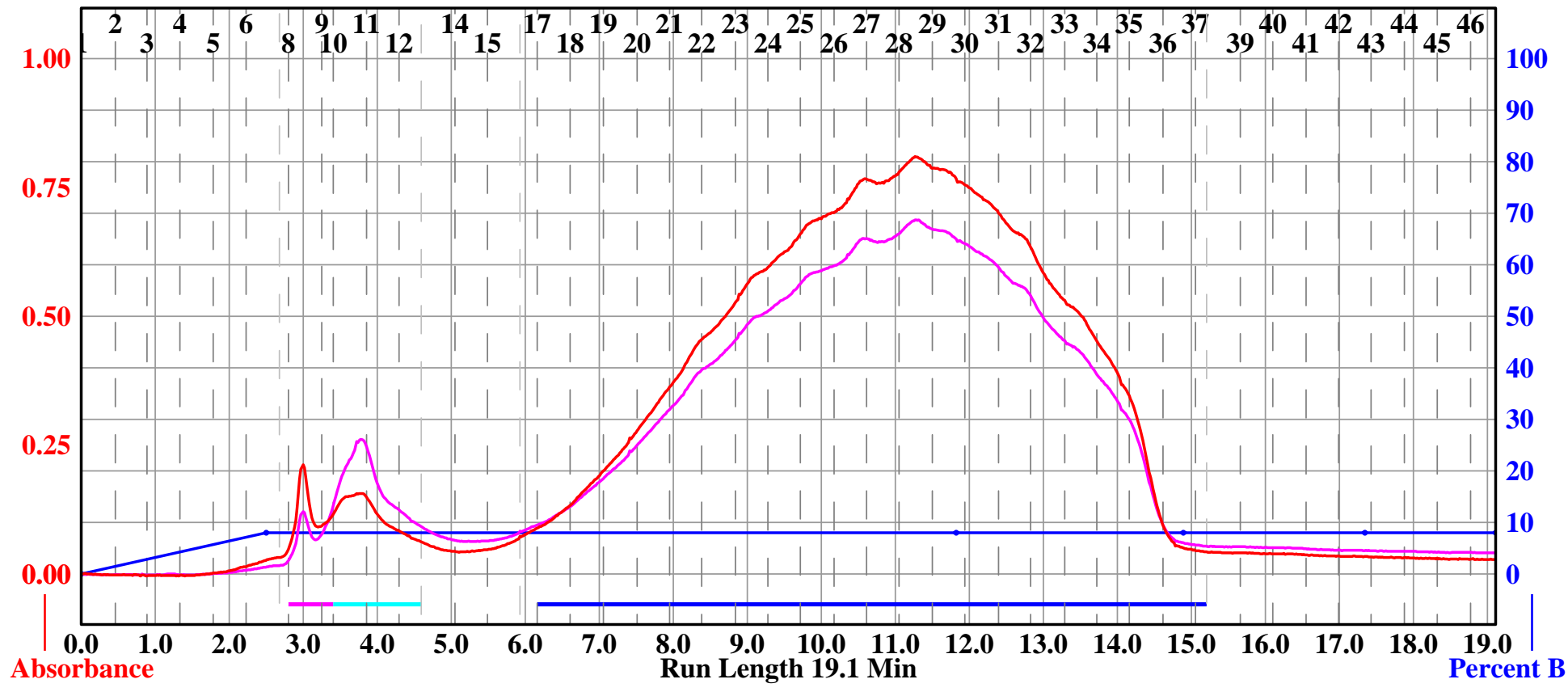

Rack A

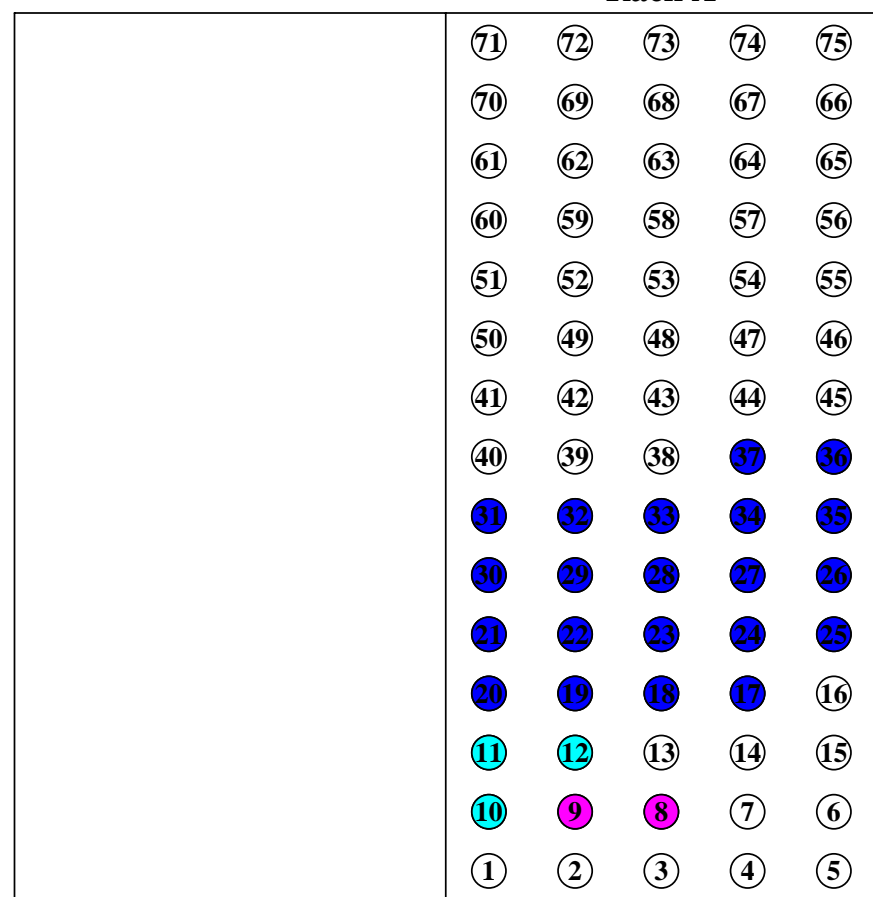

\begin{tabular}{|c|c|c|}
\hline \multicolumn{1}{|c|}{ Peak \# } & Start Tube & End Tube \\
\hline $\mathbf{1}$ & A:8 & A:9 \\
3 & A:10 & A:12 \\
& A:17 & A:37 \\
& & \\
& & \\
& & \\
& & \\
\hline
\end{tabular}

\begin{tabular}{|c|c|c|c|}
\multicolumn{1}{|c|}{ Duration } & \multicolumn{1}{c}{$\%$ B } & Solvent A & Solvent B \\
\hline $\mathbf{0 . 0}$ & $\mathbf{0 . 0}$ & A2 dichlorometha & B2 methanol \\
2.5 & $\mathbf{8 . 0}$ & A2 dichlorometha & B2 methanol \\
9.3 & $\mathbf{8 . 0}$ & A2 dichlorometha & B2 methanol \\
3.1 & $\mathbf{8 . 0}$ & A2 dichlorometha & B2 methanol \\
$\mathbf{2 . 5}$ & $\mathbf{8 . 0}$ & A2 dichlorometha & B2 methanol \\
1.8 & $\mathbf{8 . 0}$ & A2 dichlorometha & B2 methanol \\
& & & \\
& & & \\
& & & \\
& & & \\
\hline
\end{tabular}


Figure S62. 2-[6-(5,6-Diphenyl-[1,2,4]triazin-3-yl)-pyridin-2-yl]-indole-1,6-dicarboxylic acid 1-tert-butyl ester 6-methyl Ssezply9ZZG-B-193

4x Column: 4

RediSep Column: Al2O3 pH=4 8g

Flow Rate: $18 \mathrm{ml} / \mathrm{min}$

Equilibration Volume: $33.6 \mathrm{ml}$

Initial Waste: $0.0 \mathrm{ml}$

Air Purge: $1.5 \mathrm{~min}$

Solvent: A2 hexanes

Solvent: B2 (3:1) ethyl

acetate:acetone
Peak Tube Volume: $8 \mathrm{ml}$

Non-Peak Tube Volume: $8 \mathrm{ml}$

Loading Type: Solid

Wavelength 1 (red): $254 \mathrm{~nm}$

Peak Width: 30 sec

Threshold: $0.20 \mathrm{AU}$

Wavelength 2 (purple): $280 \mathrm{~nm}$

Run Notes:

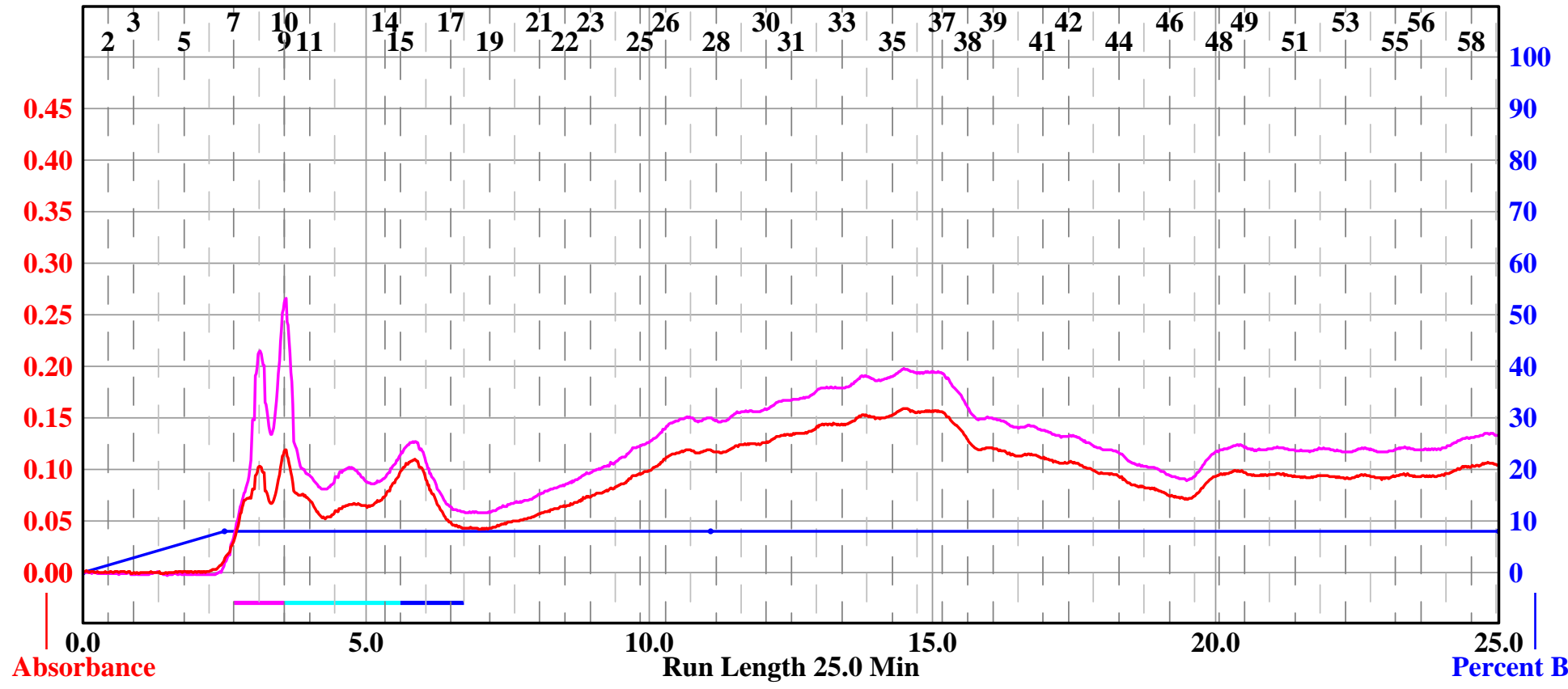

Start Tube

End Tube

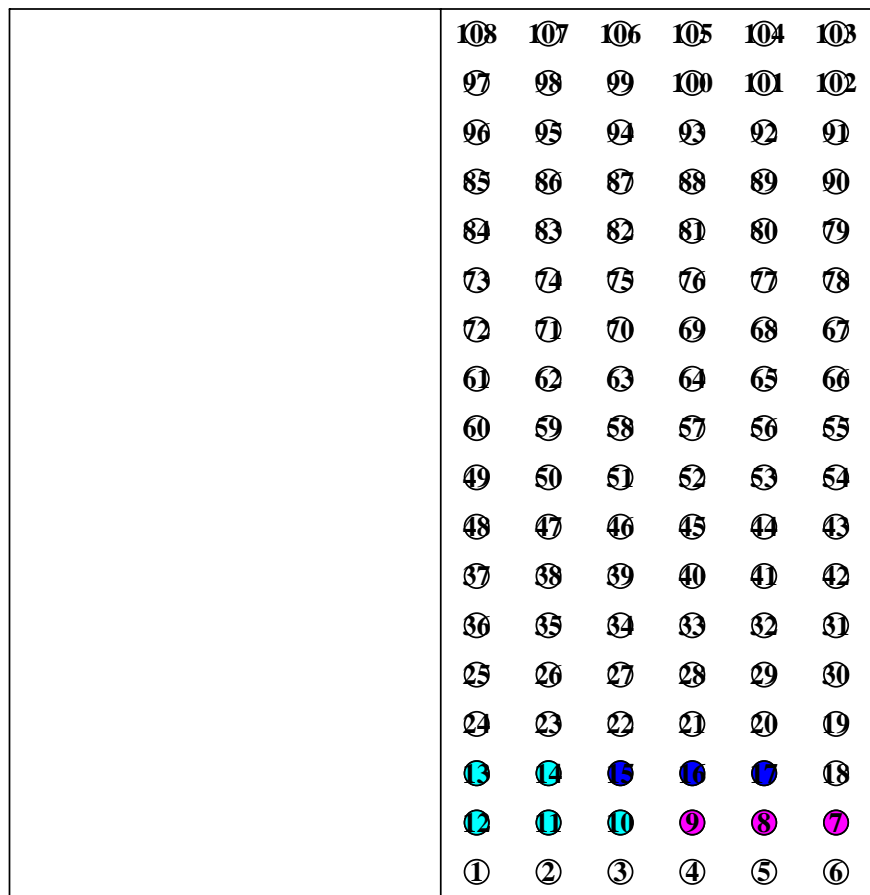

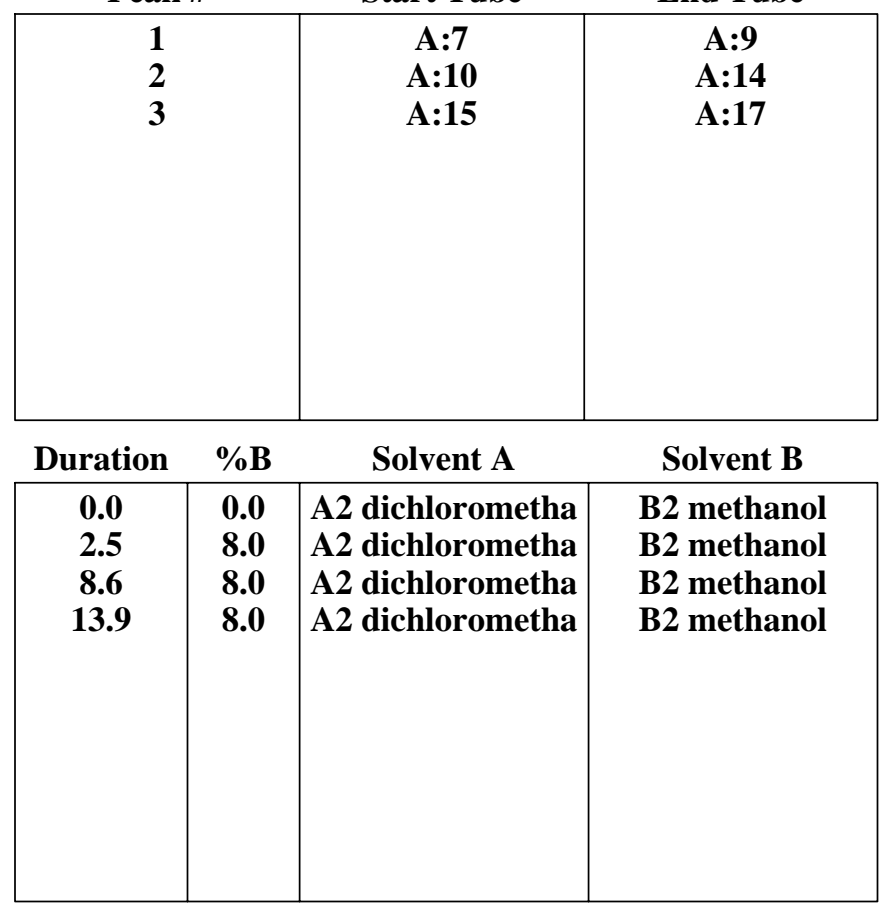


Figure S63. 2-[6-(5,6-Diphenyl-[1,2,4]triazin-3-yl)-pyridin-2-yl]-1H-indole (10) Sample: MLT-A-115

4x Column: 1

RediSep Column: Al2O3 pH=4 $8 \mathrm{~g}$

Flow Rate: $18 \mathrm{ml} / \mathrm{min}$

Equilibration Volume: $33.6 \mathrm{ml}$

Initial Waste: $0.0 \mathrm{ml}$

Air Purge: 1.5 min

Solvent: A2 hexanes

Solvent: B2 (3:1) ethyl

acetate:acetone
Peak Tube Volume: $8 \mathrm{ml}$

Non-Peak Tube Volume: $8 \mathrm{ml}$

Loading Type: Solid

Wavelength 1 (red): 254nm

Peak Width: $30 \mathrm{sec}$

Threshold: 0.20 AU

Wavelength 2 (purple): 280nm

Run Notes:

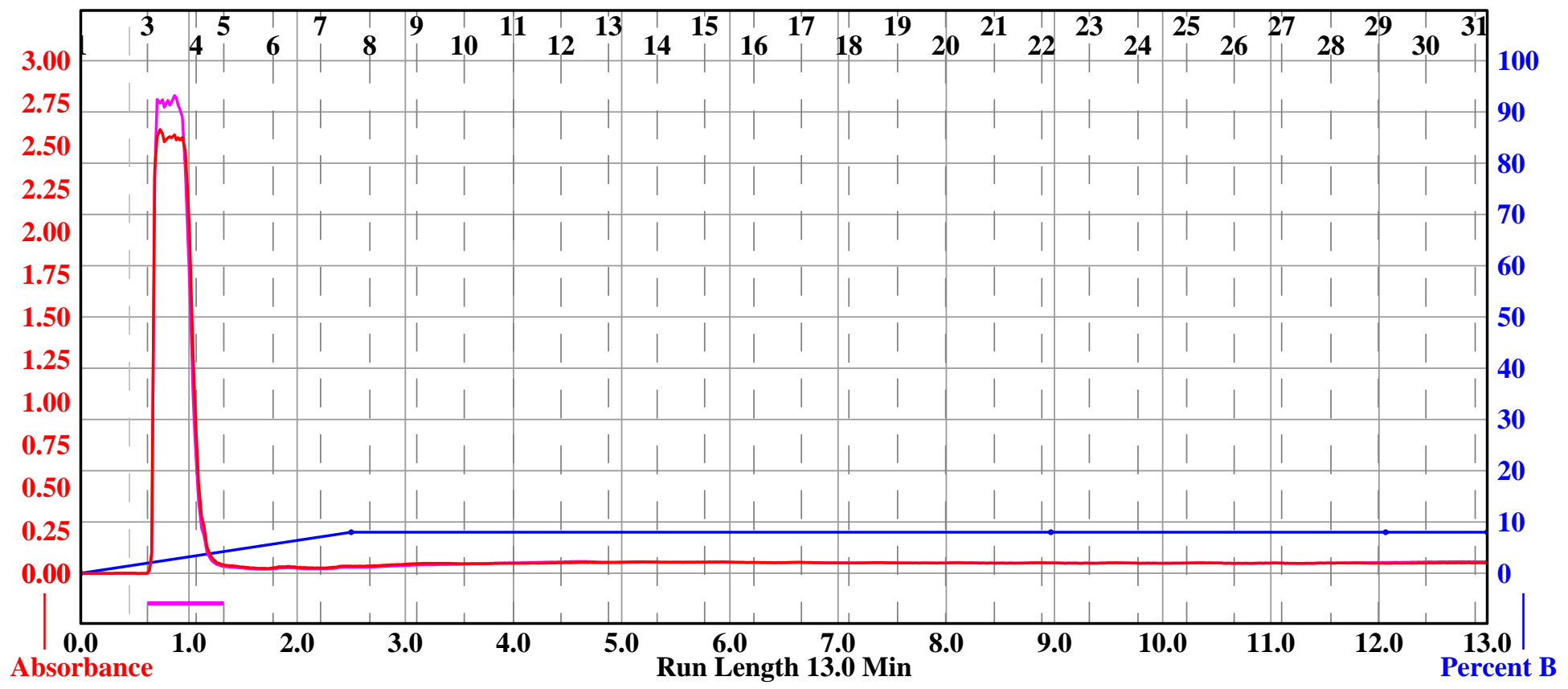

Rack A

\begin{tabular}{|c|c|c|c|c|c|}
\hline & (107) & 106 & 105 & & \\
\hline (2) & 18 & (9) & (10) & (1) & (10) \\
\hline & $\sqrt{9}$ & (9) & Q3 & (2) & (2) \\
\hline & 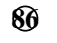 & 87 & 88 & 89 & DD \\
\hline & 83 & 82 & (11) & 80 & (19) \\
\hline & 87 & 17 & (16) & (17) & 18 \\
\hline & (11) & (10) & (9) & 68 & (67) \\
\hline & (2) & (3) & 69 & 65) & (6) \\
\hline & 59 & 68 & 57 & b & 5 \\
\hline & 50 & (1) & 52 & 53 & 54 \\
\hline & (47) & (46) & 45 & (4) & 43 \\
\hline & 18 & (39) & (41) & (41) & (42) \\
\hline & BF & B9 & B3 & (32) & (1) \\
\hline & שூ & (27) & 8 & (29) & (10) \\
\hline & 23 & (2) & (2) & (21) & (19) \\
\hline & $\mathbb{1}$ & (15) & (16) & (1) & $\mathbb{1 8}$ \\
\hline & (11) & (10) & (2) & (8) & (7) \\
\hline & & & & ( & \\
\hline
\end{tabular}

$13 \mathrm{~mm} \times 100 \mathrm{~mm}$ Tubes

\begin{tabular}{|c|c|c|c|}
\hline \multicolumn{2}{|c|}{ Peak \# } & Start Tube & End Tube \\
\hline \multicolumn{2}{|c|}{1} & $\mathbf{A}: \mathbf{3}$ & \multirow[b]{2}{*}{ Solvent B } \\
\hline Duration & $\% \mathbf{B}$ & Solvent A & \\
\hline 0.0 & 0.0 & A2 dichlorometha & B2 methanol \\
\hline 2.5 & 8.0 & A2 dichlorometha & B2 methanol \\
\hline 6.5 & 8.0 & A2 dichlorometha & B2 methanol \\
\hline 3.1 & 8.0 & A2 dichlorometha & B2 methanol \\
\hline 0.9 & 8.0 & A2 dichlorometha & B2 methanol \\
\hline & & & \\
\hline & & & \\
\hline & & & \\
\hline
\end{tabular}


Figure S64. 3-(6-Benzofuran-2-yl-pyridin-2-yl)-5,6-diphenyl-[1,2,4] triazine (11) Sample: ZZG-B-227

4x Column: 4

RediSep Column: $\mathrm{Al}_{2} \mathrm{O}_{3}$,

$\mathrm{pH}=4,8 \mathrm{~g}$

Flow Rate: $18 \mathrm{ml} / \mathrm{min}$

Equilibration Volume: $33.6 \mathrm{ml}$

Initial Waste: $0.0 \mathrm{ml}$

Air Purge: $1.0 \mathrm{~min}$

Solvent: A2 hexanes

Solvent: B1 ethyl acetate
Peak Tube Volume: $8 \mathrm{ml}$

Non-Peak Tube Volume: $8 \mathrm{ml}$

Loading Type: Solid

Wavelength 1 (red): 254nm

Peak Width: 30 sec

Threshold: 0.20 AU

Wavelength 2 (purple): $365 \mathrm{~nm}$

Run Notes:

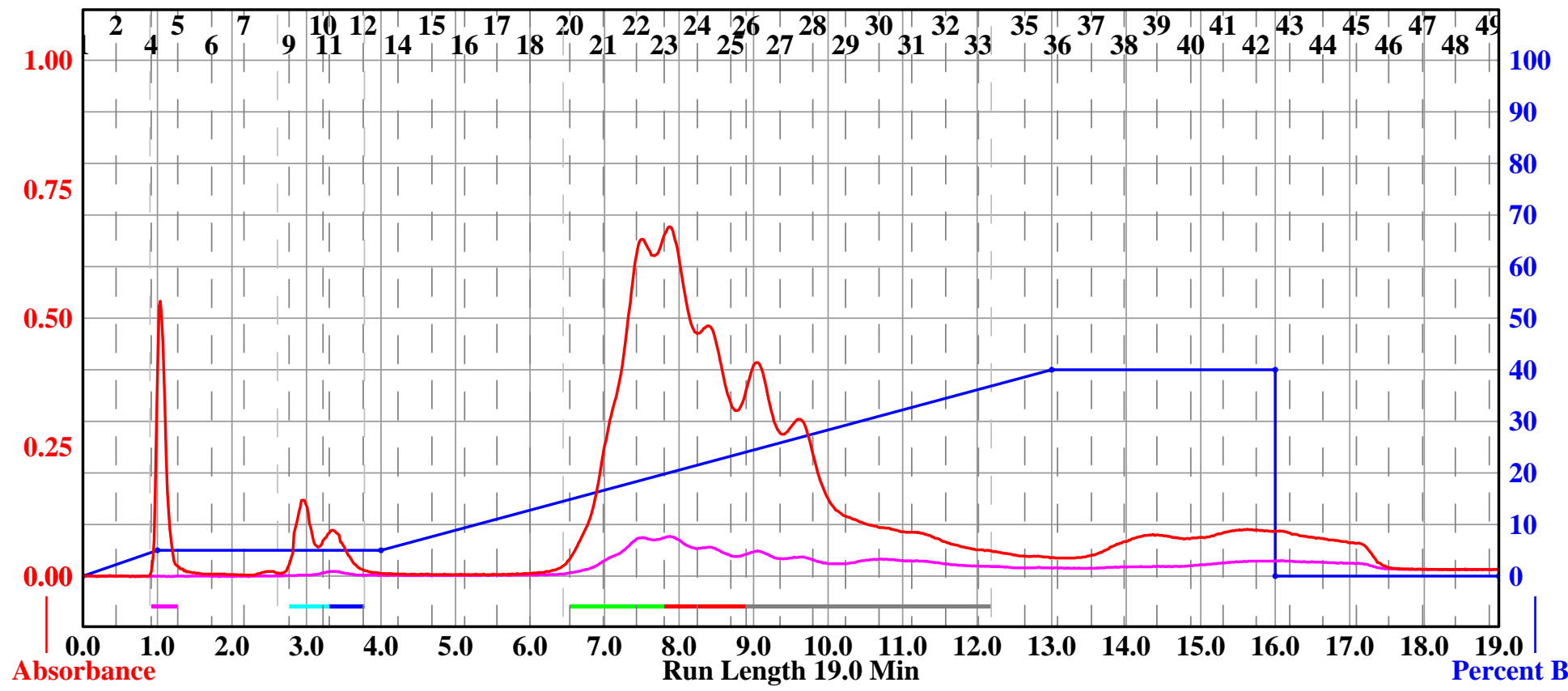

Rack A

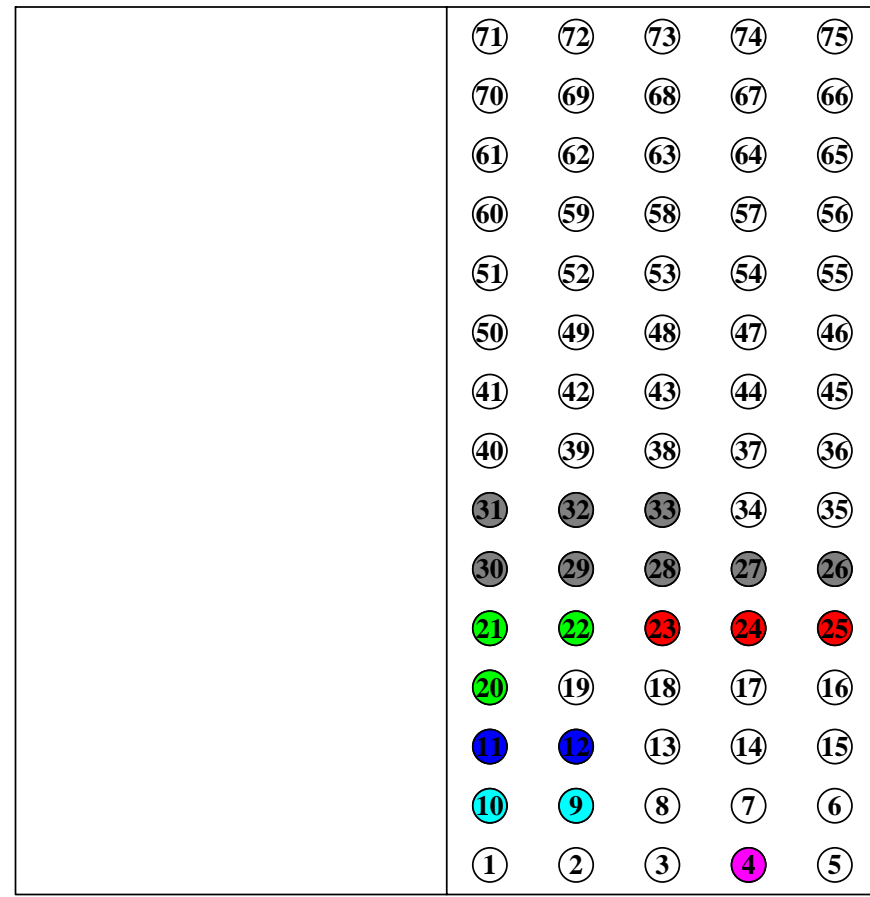

Peak \# $\quad$ Start Tube $\quad$ End Tube

\begin{tabular}{|c|c|c|c|}
\hline $\begin{array}{l}1 \\
2 \\
2 \\
3 \\
4 \\
5 \\
5 \\
6\end{array}$ & & $\begin{array}{c}\text { A:4 } \\
A: 9 \\
A: 11 \\
A: 20 \\
A: 23 \\
A: 26\end{array}$ & $\begin{array}{c}\text { A:4 } \\
A: 10 \\
A: 12 \\
A: 22 \\
A: 25 \\
A: 33\end{array}$ \\
\hline Duration & $\% \mathbf{B}$ & Solvent A & Solvent B \\
\hline $\begin{array}{l}\text { 0.0 } \\
\text { 1.0 } \\
\text { 3.0 } \\
\text { 9.0 } \\
\text { 3.0 } \\
\text { 0.0 } \\
\text { 3.0 }\end{array}$ & $\begin{array}{c}0.0 \\
5.0 \\
5.0 \\
40.0 \\
40.0 \\
0.0 \\
0.0\end{array}$ & $\begin{array}{l}\text { A2 dichlorometha } \\
\text { A2 dichlorometha } \\
\text { A2 dichlorometha } \\
\text { A2 dichlorometha } \\
\text { A2 dichlorometha } \\
\text { A2 dichlorometha } \\
\text { A2 dichlorometha }\end{array}$ & $\begin{array}{l}\text { B1 ethyl acetate } \\
\text { B1 ethyl acetate } \\
\text { B1 ethyl acetate } \\
\text { B1 ethyl acetate } \\
\text { B1 ethyl acetate } \\
\text { B1 ethyl acetate } \\
\text { B1 ethyl acetate }\end{array}$ \\
\hline
\end{tabular}


Figure S65. 2-[6-(5,6-Di-p-tolyl-[1,2,4]triazin-3-yl)-pyridin-2-yl]-4-methyl-indole-1-carboxylic acid tert-butyl ester (13) Sample: MLT-A-131

4x Column: 1

RediSep Column: Al2O3 pH=4 8g

Flow Rate: $18 \mathrm{ml} / \mathrm{min}$

Equilibration Volume: $33.6 \mathrm{ml}$

Initial Waste: $0.0 \mathrm{ml}$

Air Purge: 1.5 min

Solvent: A2 hexanes

Solvent: B2 (3:1) ethyl

acetate:acetone
Peak Tube Volume: Max.

Non-Peak Tube Volume: Max.

Loading Type: Solid

Wavelength 1 (red): 254nm

Peak Width: $30 \mathrm{sec}$

Threshold: 0.20 AU

Wavelength 2 (purple): 280nm

Run Notes:

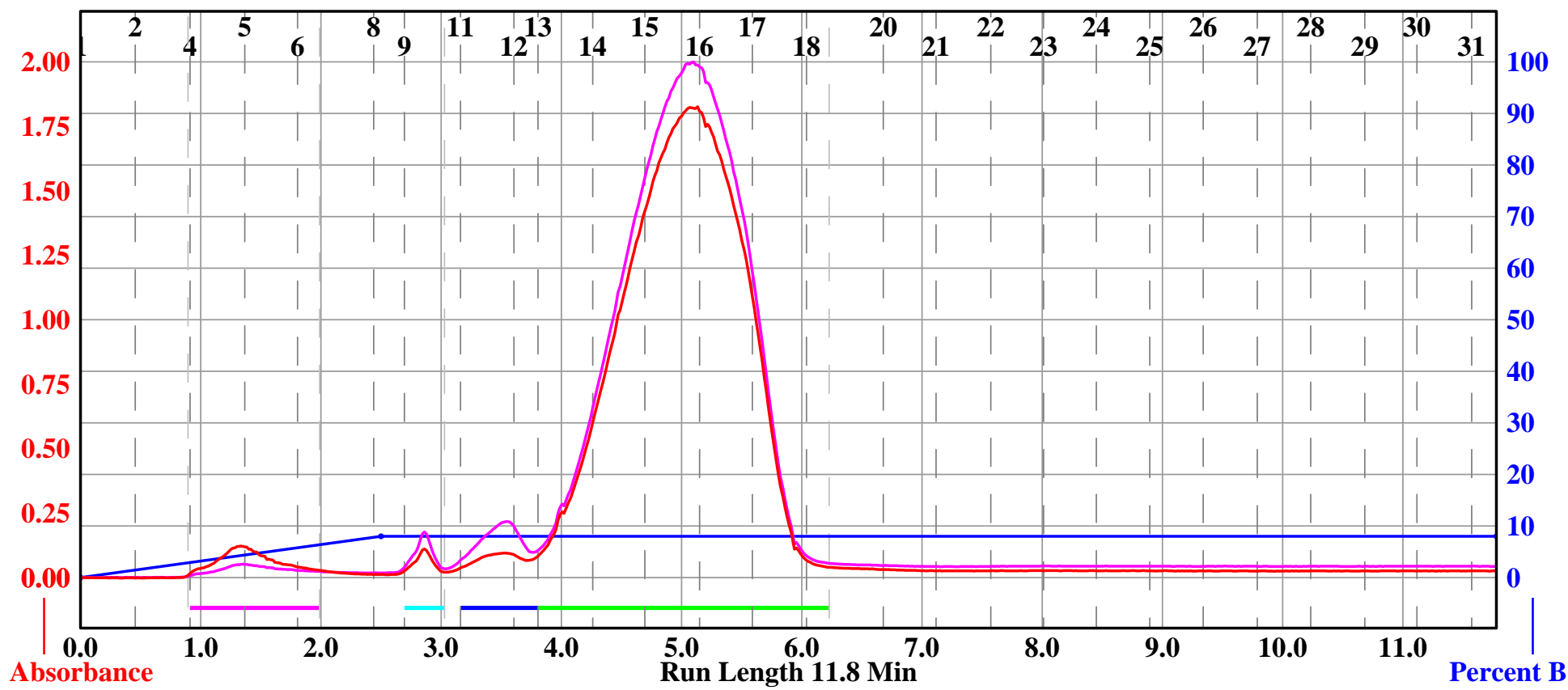

Rack A

\begin{tabular}{|c|c|c|c|c|c|}
\hline 18 & (107) & (106 & (105 & (1)4 & 103 \\
\hline (27) & 18 & (99 & 100 & (1)1 & (102 \\
\hline 26 & (93) & (9) & (3) & (2) & (2) \\
\hline 85 & 8 & 87 & 88 & 89 & (D) \\
\hline 89 & 83 & 82 & (1) & (810 & (7) \\
\hline 13 & (27) & 87 & (80) & (77) & 88 \\
\hline (2) & (11) & (1) & (9) & 68 & (67) \\
\hline (1) & (2) & 63 & (4) & 6 & (6) \\
\hline (1) & 59 & 68 & 57 & שd & 55 \\
\hline (49) & (1) & (1) & 52 & 63 & 59 \\
\hline 48 & (47) & (46) & 49 & (4) & (43) \\
\hline (37) & 18 & (39) & (41) & (41) & (42) \\
\hline (36) & BF & (3) & B3 & (22) & (1) \\
\hline (25 & 20 & 87 & 88 & (29) & (31) \\
\hline (2) & 3 & 22 & (1) & (21) & (1) \\
\hline (B) & (1) & (1) & (16) & (1) & (18) \\
\hline 0 & 0 & (II) & (D) & (8) & (7) \\
\hline (1) & (2) & (3) & (4) & (5) & (6) \\
\hline
\end{tabular}

$13 \mathrm{~mm} \times 100 \mathrm{~mm}$ Tubes

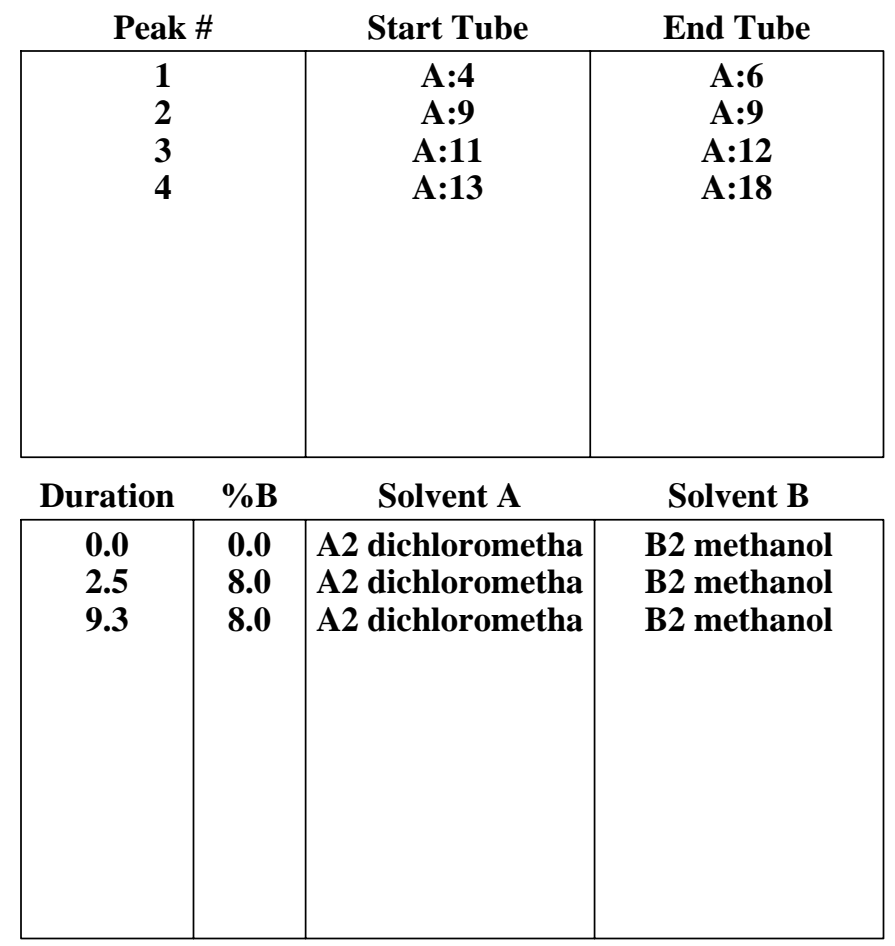


Figure S66. 2-[6-(5,6-Di-p-tolyl-[1,2,4]triazin-3-yl)-pyridin-2-yl]-indole-1-carboxylic acid tert-butyl ester (14) Sample: MLT-A-179

4x Column: 4

RediSep Column: Al2O3 pH=4 8g

Flow Rate: $18 \mathrm{ml} / \mathrm{min}$

Equilibration Volume: $33.6 \mathrm{ml}$

Initial Waste: $0.0 \mathrm{ml}$

Air Purge: $0.5 \mathrm{~min}$

Solvent: A2 hexanes

Solvent: B2 (3:1) ethyl

acetate:acetone
Peak Tube Volume: Max.

Non-Peak Tube Volume: Max.

Loading Type: Solid

Wavelength 1 (red): 254nm

Peak Width: $30 \mathrm{sec}$

Threshold: 0.20 AU

Wavelength 2 (purple): 280nm

Run Notes:

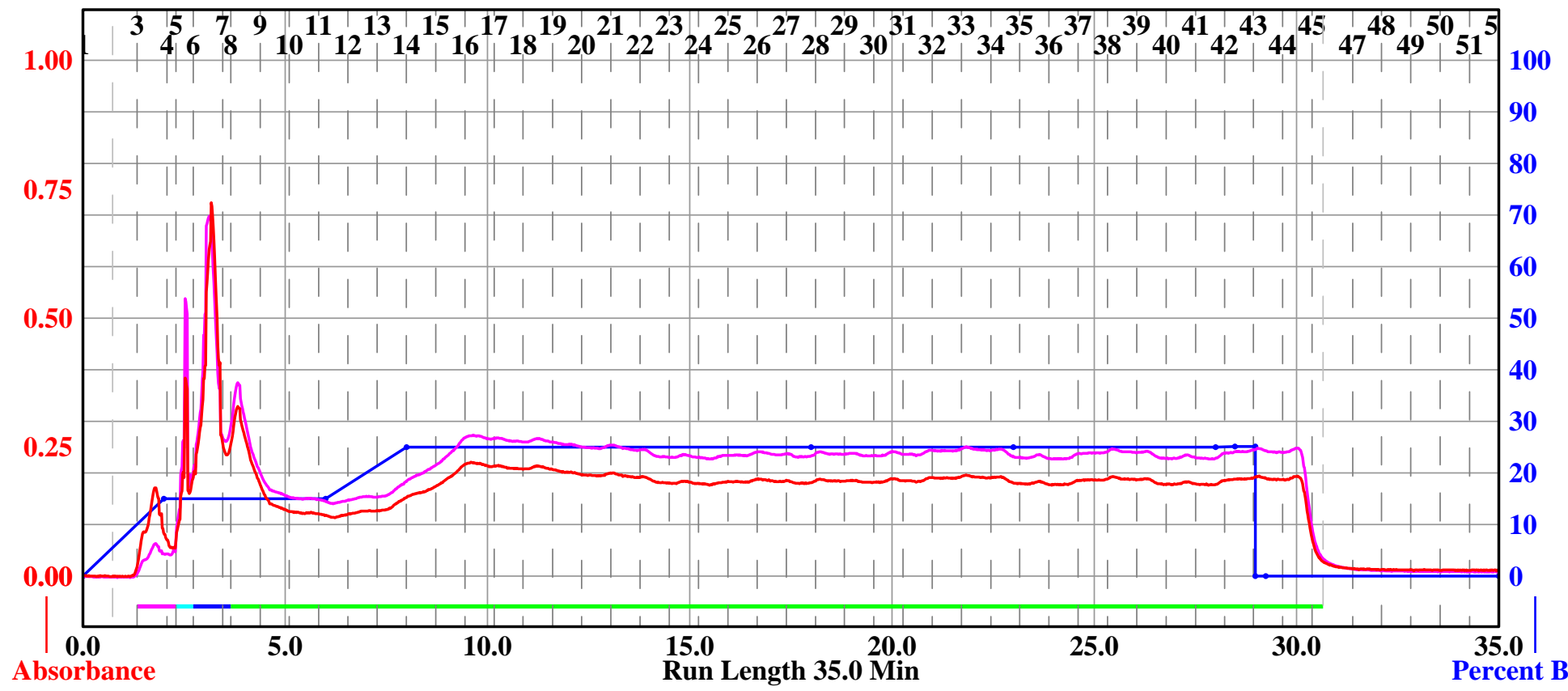

Rack A

\begin{tabular}{|c|c|c|c|}
\hline (11) & (72) & (73) & (74) \\
\hline (70) & (69) & (68) & (67) \\
\hline (11) & (62) & (63) & (44) \\
\hline (60) & (99) & (58) & (57) \\
\hline (51) & (52) & (33) & (54) \\
\hline (50) & (49) & (48) & (47) \\
\hline (411) & (42) & (43) & (44) \\
\hline (410) & (39) & (38) & (37) \\
\hline (31) & (32) & (33) & (34) \\
\hline (30) & (29) & (28) & (27) \\
\hline (21) & (22) & (23) & (24) \\
\hline (20) & (19) & (18) & (17) \\
\hline (11) & (12) & (13) & (14) \\
\hline (10) & (9) & (8) & \\
\hline (1) & (2) & (3) & (4) \\
\hline
\end{tabular}

$16 \mathrm{~mm} \times 100 \mathrm{~mm}$ Tubes

\begin{tabular}{|c|c|c|c|}
\hline \multicolumn{2}{|c|}{ Peak \# } & Start Tube & End Tube \\
\hline $\begin{array}{l}1 \\
2 \\
3 \\
4\end{array}$ & & $\begin{array}{l}\text { A:3 } \\
\text { A:5 } \\
\text { A:6 } \\
\text { A:8 }\end{array}$ & $\begin{array}{c}\text { A:4 } \\
\text { A:5 } \\
\text { A:7 } \\
\text { A:45 }\end{array}$ \\
\hline Duration & $\% \mathbf{B}$ & Solvent A & Solvent B \\
\hline 0.0 & 0.0 & A2 dichlorometha & B2 methanol \\
\hline 2.0 & 15.0 & A2 dichlorometha & B2 methanol \\
\hline 4.0 & 15.0 & A2 dichlorometha & B2 methanol \\
\hline 2.0 & 25.0 & A2 dichlorometha & B2 methanol \\
\hline 10.0 & 25.0 & A2 dichlorometha & B2 methanol \\
\hline 5.0 & 25.0 & A2 dichlorometha & B2 methanol \\
\hline 5.0 & 25.0 & A2 dichlorometha & B2 methanol \\
\hline 0.5 & 25.1 & A2 dichlorometha & B2 methanol \\
\hline 0.5 & 25.1 & A2 dichlorometha & B2 methanol \\
\hline $\begin{array}{l}0.0 \\
\ldots . .0\end{array}$ & $\begin{array}{l}0.0 \\
\ldots . .\end{array}$ & A2 dichlorometha & B2 methanol \\
\hline
\end{tabular}


Figure S67. 2-\{6-[5,6-Bis-(4-fluoro-phenyl)-[1,2,4]triazin-3-yl]-pyridin-2-yl\}-5-fluoro-indole-1-carboxylic acid tert-butyl Sรteplei Z7) G-B-179

4x Column: 1

RediSep Column: Al2O3 pH=4 8g

Flow Rate: $18 \mathrm{ml} / \mathrm{min}$

Equilibration Volume: $33.6 \mathrm{ml}$

Initial Waste: $0.0 \mathrm{ml}$

Air Purge: 1.0 min

Solvent: A2 hexanes

Solvent: B2 (3:1) ethyl

acetate:acetone
Peak Tube Volume: Max.

Non-Peak Tube Volume: Max.

Loading Type: Solid

Wavelength 1 (red): 254nm

Peak Width: 30 sec

Threshold: 0.20 AU

Wavelength 2 (purple): 280nm

Run Notes:

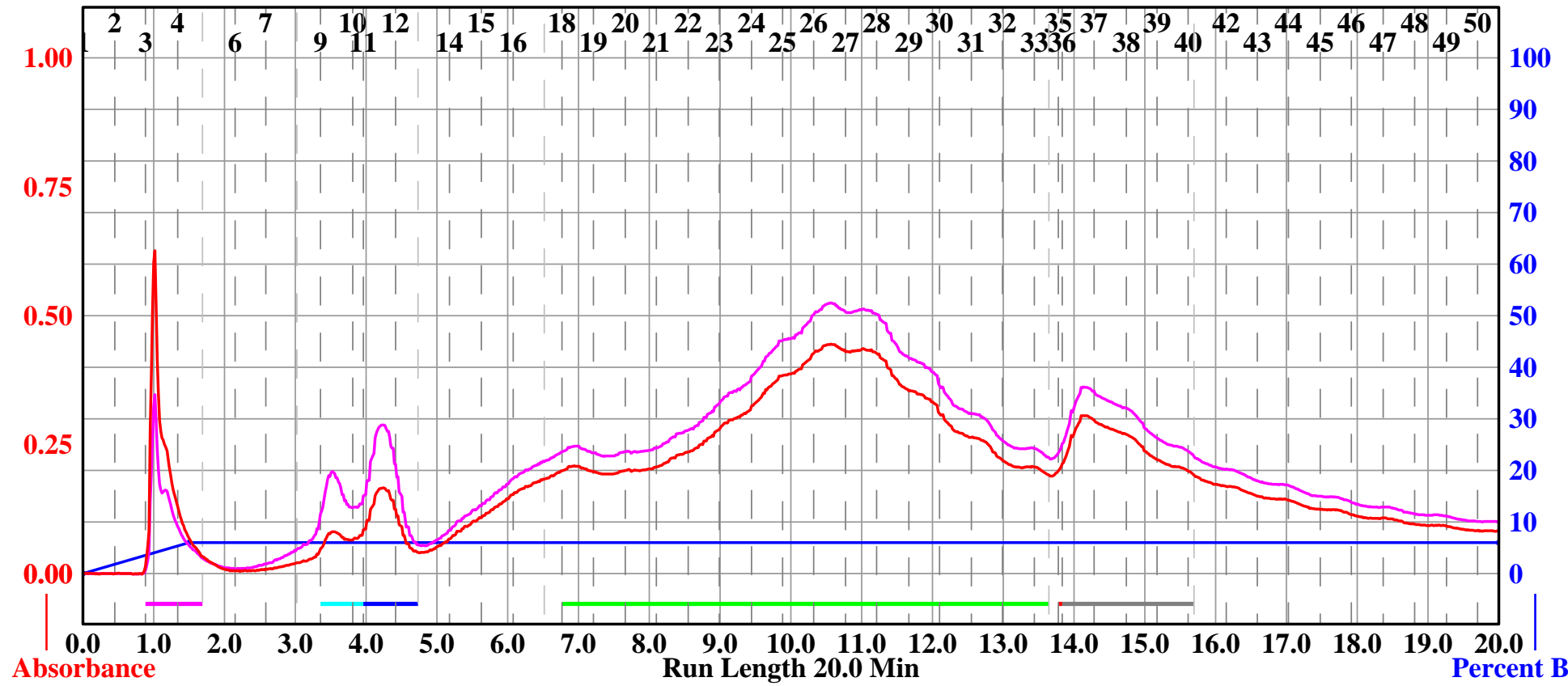

Rack A

Peak \#

Start Tube

End Tube

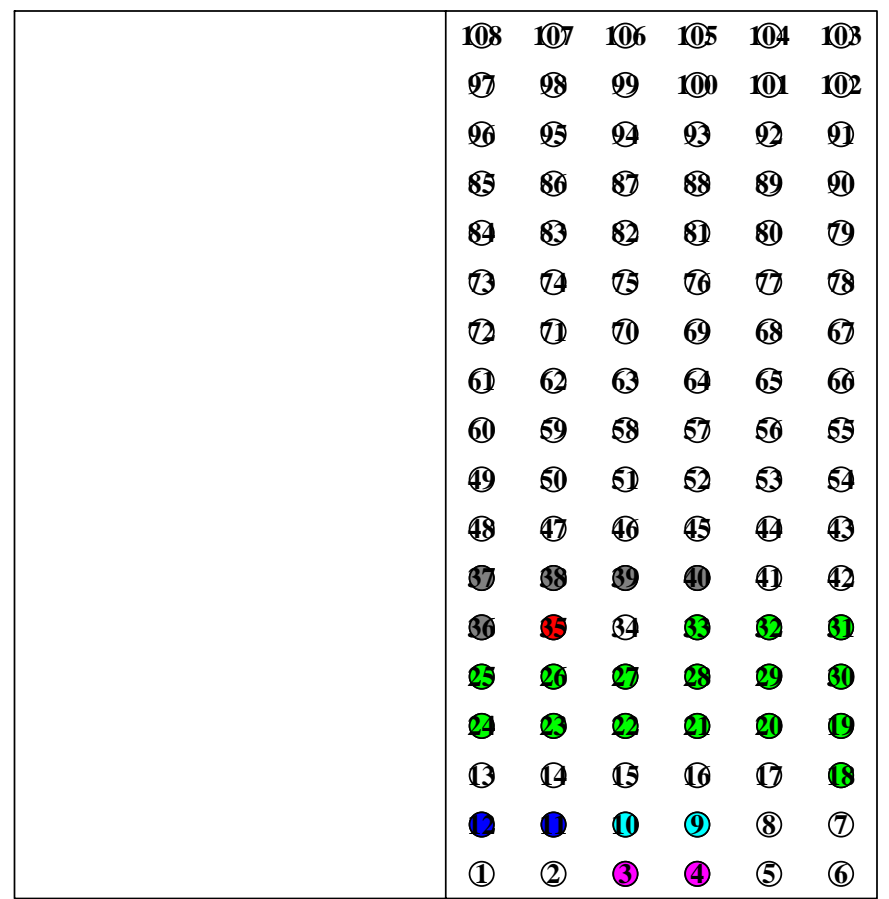

$13 \mathrm{~mm} \times 100 \mathrm{~mm}$ Tubes

\begin{tabular}{|c|c|c|c|}
\hline $\begin{array}{l}1 \\
2 \\
3 \\
4 \\
5 \\
6\end{array}$ & & $\begin{array}{c}\mathbf{A}: 3 \\
\mathbf{A}: 9 \\
\mathbf{A}: 11 \\
\mathbf{A}: 18 \\
\mathbf{A}: 35 \\
\mathbf{A}: 36\end{array}$ & $\begin{array}{c}\mathbf{A}: 4 \\
\mathbf{A}: 10 \\
\mathbf{A}: 12 \\
\mathbf{A}: 33 \\
\mathbf{A}: 35 \\
\mathbf{A}: 40\end{array}$ \\
\hline Duration & $\% B$ & Solvent A & Solvent B \\
\hline $\begin{array}{c}0.0 \\
1.5 \\
18.5\end{array}$ & $\begin{array}{l}0.0 \\
6.0 \\
6.0\end{array}$ & $\begin{array}{l}\text { A2 dichlorometha } \\
\text { A2 dichlorometha } \\
\text { A2 dichlorometha }\end{array}$ & $\begin{array}{l}\text { B2 methanol } \\
\text { B2 methanol } \\
\text { B2 methanol }\end{array}$ \\
\hline
\end{tabular}


Figure S68. 2-\{6-[5,6-Bis-(4-fluoro-phenyl)-[1,2,4]triazin-3-yl]-pyridin-2-yl\}-5-(tert-butyl-dimethyl-silanyloxy)-indole-1carboxylic acid tert-butyl ester (16) Sample: ZZG-B-181

Rf 200

Friday 01 March 2019 03:40PM

Peak Tube Volume: Max.

Non-Peak Tube Volume: Max.

Loading Type: Solid

Wavelength 1 (red): 254nm

Peak Width: 30 sec

Threshold: 0.20 AU

Wavelength 2 (purple): 280nm

Run Notes:

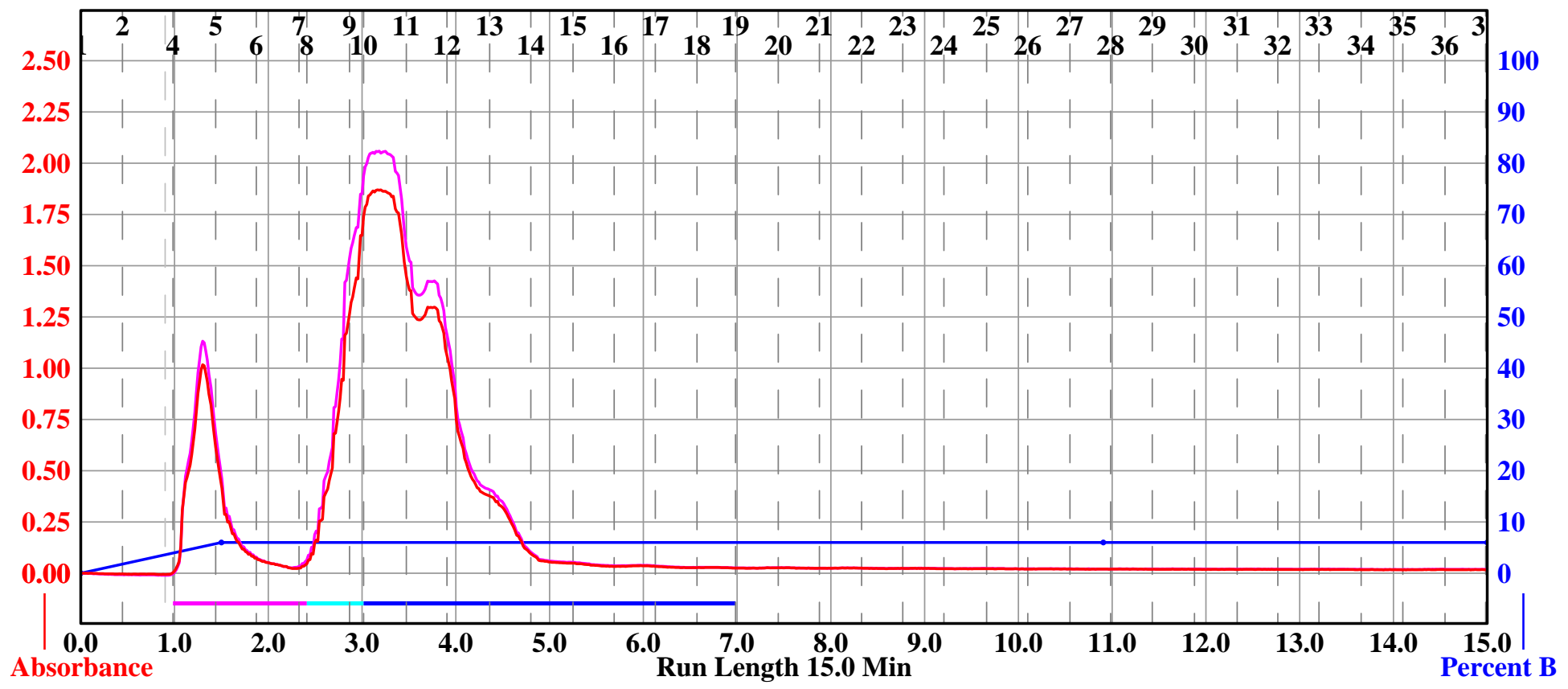

Rack A

Peak \#

Start Tube

End Tube

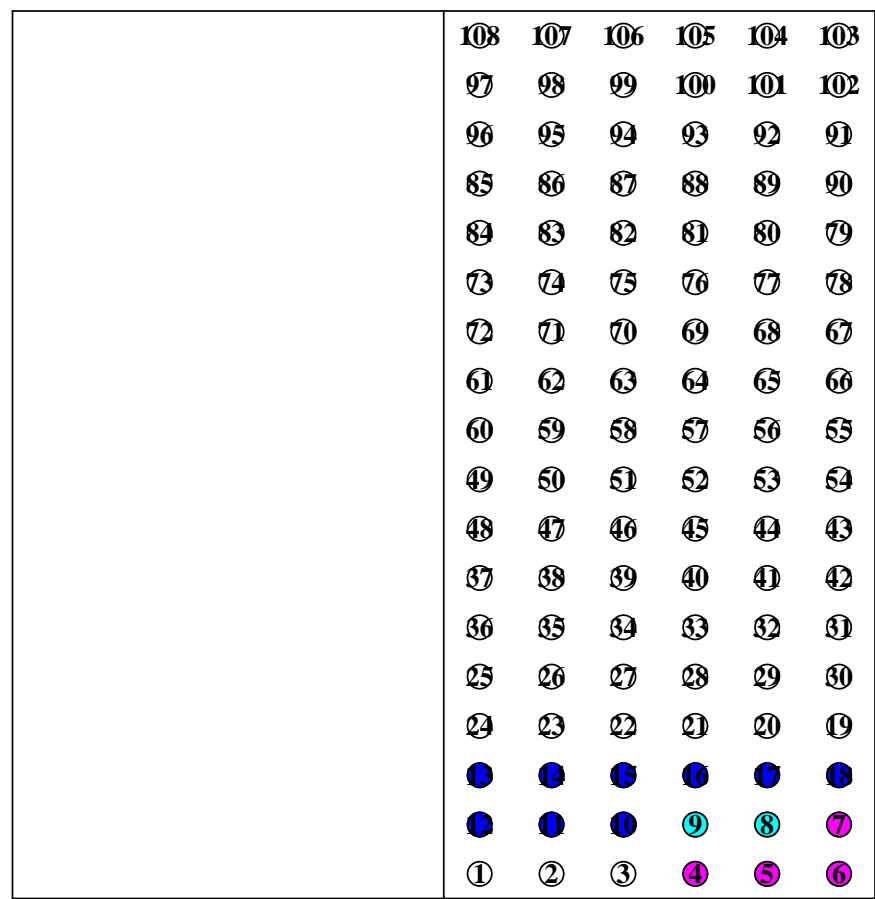

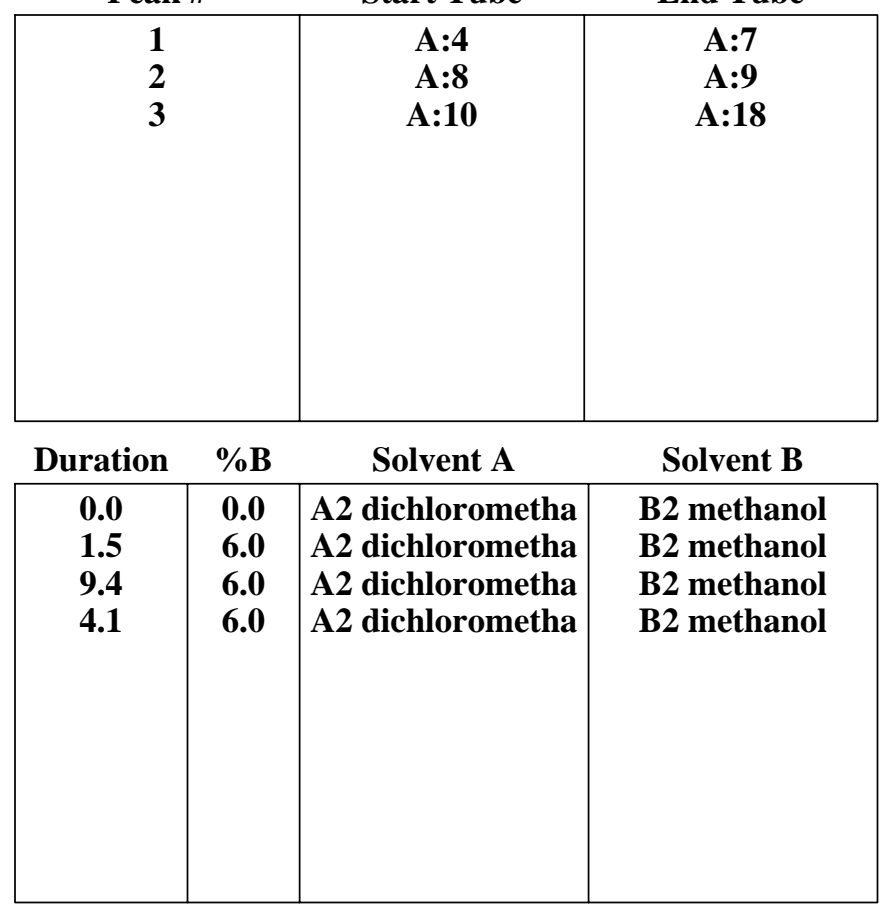


Figure S69. 2-\{6-[5,6-Bis-(3-methoxy-phenyl)-[1,2,4]triazin-3-yl]-pyridin-2-yl\}-5-methoxy-indole-1-carbox-ylic acid tertbutyl ester (17)

Sample: ZZG-B-189

\section{Rf 200}

Thursday 14 March 2019 01:01PM

4x Column: 3

RediSep Column: Al2O3 pH=7 8g

Flow Rate: $18 \mathrm{ml} / \mathrm{min}$

Equilibration Volume: $33.6 \mathrm{ml}$

Initial Waste: $0.0 \mathrm{ml}$

Air Purge: $0.5 \mathrm{~min}$

Solvent: A2 hexanes

Solvent: B2 (3:1) ethyl

acetate:acetone
Peak Tube Volume: $8 \mathrm{ml}$

Non-Peak Tube Volume: $8 \mathrm{ml}$

Loading Type: Solid

Wavelength 1 (red): 254nm

Peak Width: 30 sec

Threshold: 0.20 AU

Wavelength 2 (purple): 280nm

Run Notes:

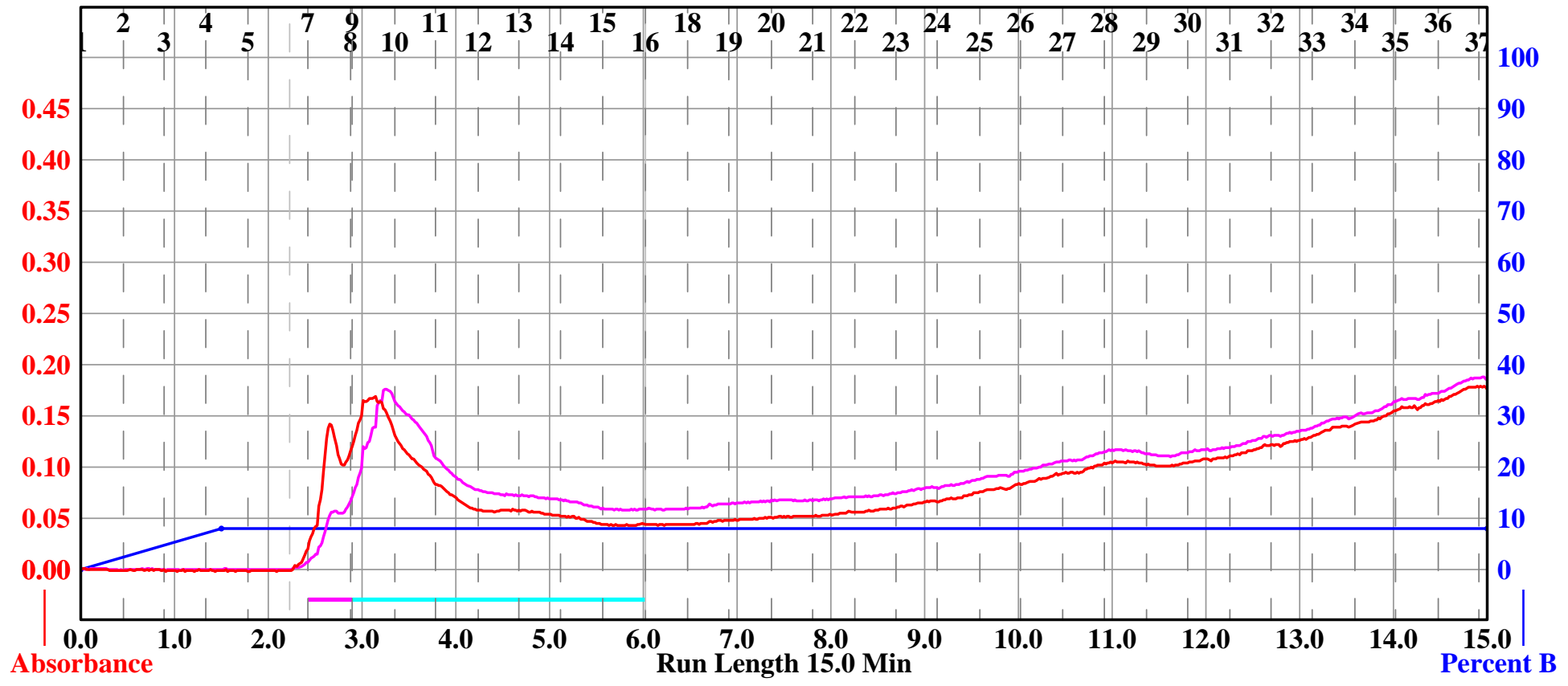

Rack A

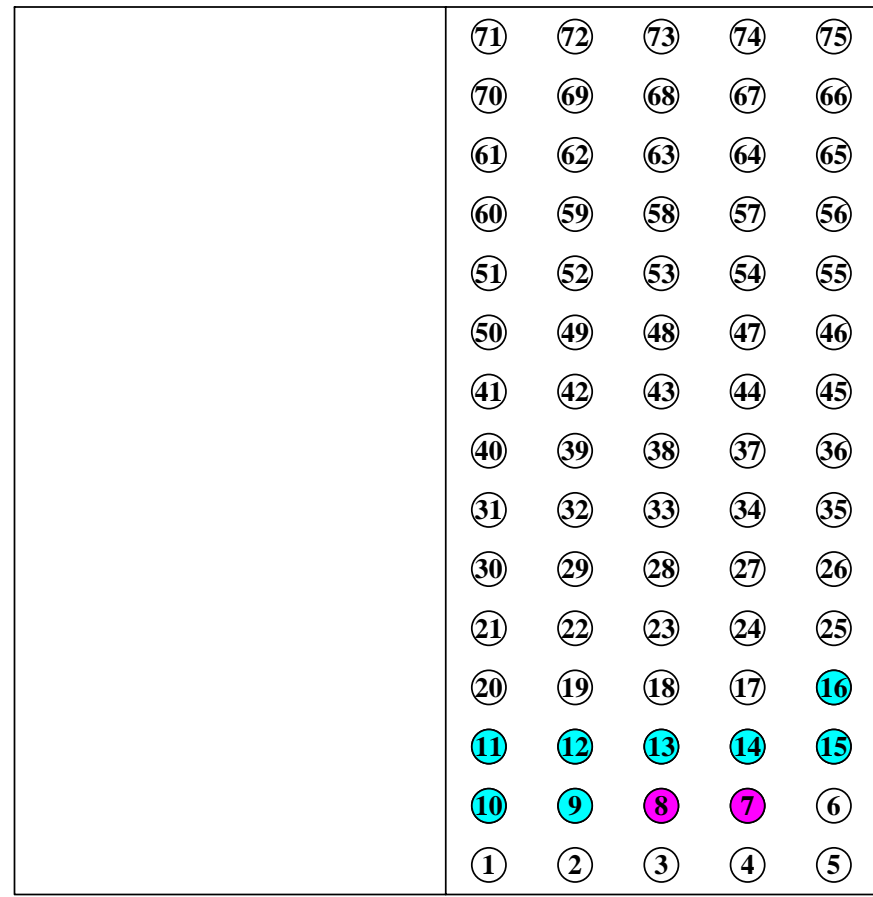

Start Tube

End Tube

Peak \#

A:7

A:9

A:8

A:16

\begin{tabular}{|c|c|c|}
\hline 1 & A:7 & A:8 \\
2 & A:9 & A:16 \\
& & \\
& & \\
& & \\
& & \\
\hline
\end{tabular}

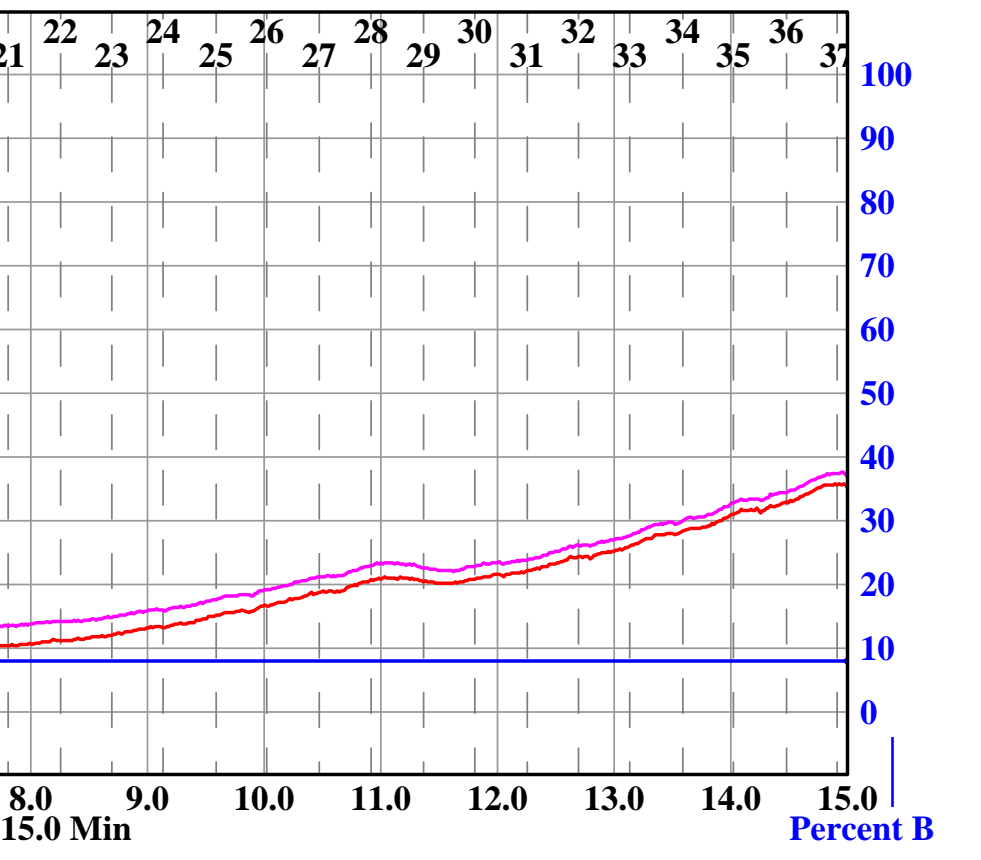

\begin{tabular}{|c|c|c|c|}
\hline 0.0 & 0.0 & A2 dichlorometha & B2 methanol \\
1.5 & 8.0 & A2 dichlorometha & B2 methanol \\
13.5 & $\mathbf{8 . 0}$ & A2 dichlorometha & B2 methanol \\
& & & \\
& & & \\
& & & \\
& & & \\
\hline
\end{tabular}


Figure S70. 2-\{6-[5,6-Bis-(4-cyclopropyl-phenyl)-[1,2,4]triazin-3-yl]-pyridin-2-yl\}-4-methyl-indole-1-carboxylic acid tertbutyl ester (18)

Sample: ZZG-B-163

Rf 200

Wednesday 27 February 2019 12:19PM

4x Column: 1

RediSep Column: Al2O3 pH=4 24g

Flow Rate: $30 \mathrm{ml} / \mathrm{min}$

Equilibration Volume: $79.2 \mathrm{ml}$

Initial Waste: $0.0 \mathrm{ml}$

Air Purge: $0.5 \mathrm{~min}$

Solvent: A2 hexanes

Peak Tube Volume: $8 \mathrm{ml}$

Non-Peak Tube Volume: $8 \mathrm{ml}$

Loading Type: Solid

Wavelength 1 (red): 254nm

Peak Width: 1 min

Threshold: 0.20 AU

Solvent: B2 (3:1) ethyl

Wavelength 2 (purple): 280nm

acetate:acetone

Run Notes:

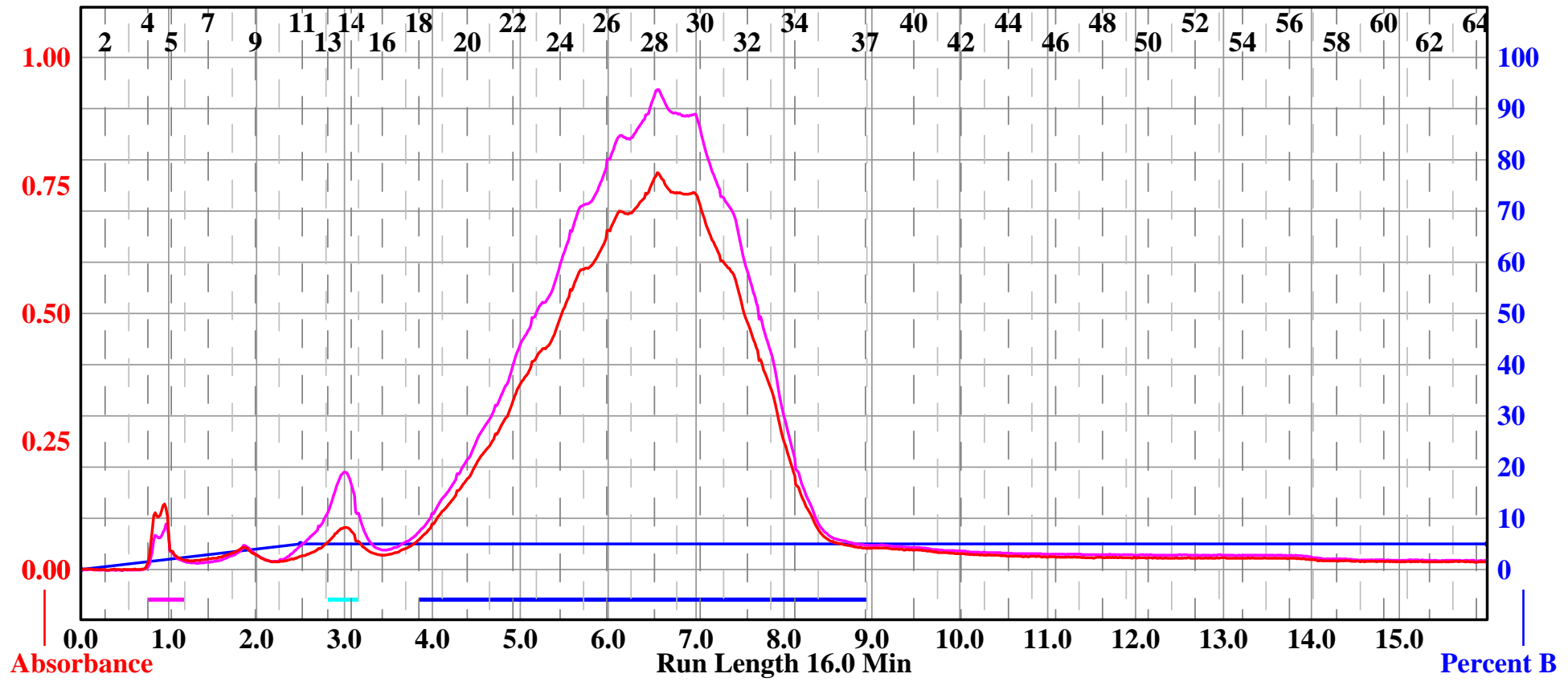

Rack A

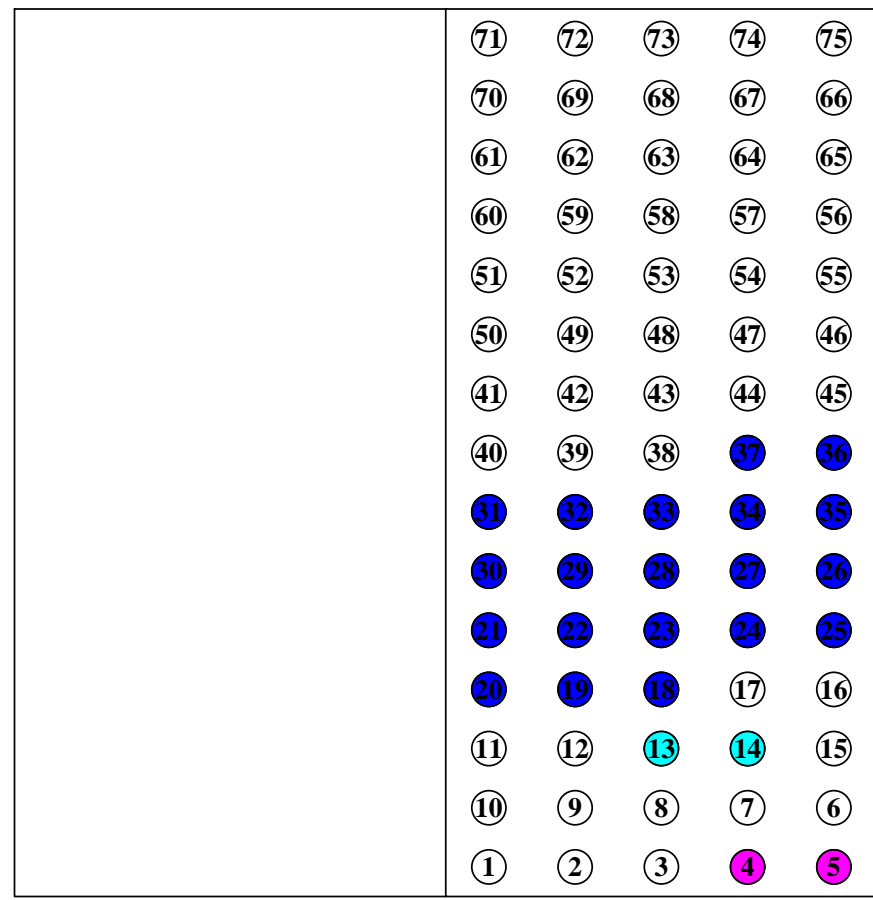

$16 \mathrm{~mm} \times 100 \mathrm{~mm}$ Tubes

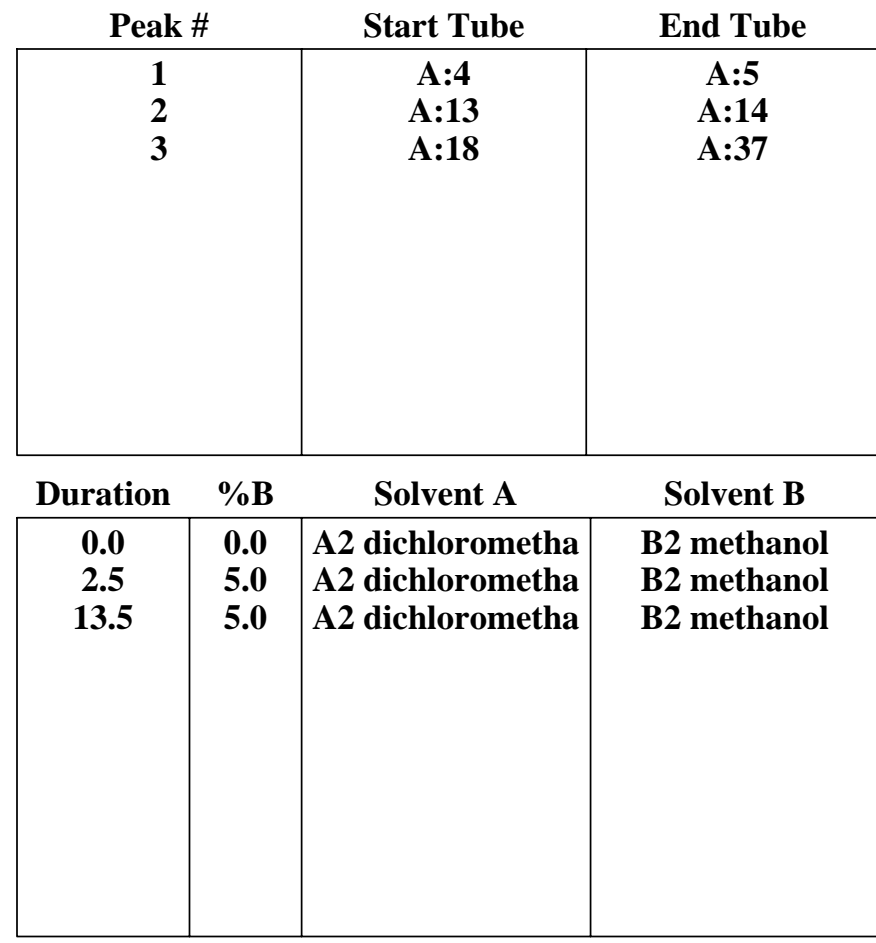


Figure S71. 2-\{6-[5,6-Bis-(4-cyclopropyl-phenyl)-[1,2,4]triazin-3-yl]-pyridin-2-yl\}-1H-indole (19)

Sample: ZZG-B-165

Rf 200

Wednesday 27 February 2019 06:10PM

4x Column: 3

RediSep Column: Al2O3 pH=4 8g

Flow Rate: $18 \mathrm{ml} / \mathrm{min}$

Equilibration Volume: $33.6 \mathrm{ml}$

Initial Waste: $0.0 \mathrm{ml}$

Air Purge: $0.5 \mathrm{~min}$

Solvent: A2 hexanes

Solvent: B2 (3:1) ethyl

acetate:acetone
Peak Tube Volume: Max.

Non-Peak Tube Volume: Max.

Loading Type: Solid

Wavelength 1 (red): $254 \mathrm{~nm}$

Peak Width: 30 sec

Threshold: 0.20 AU

Wavelength 2 (purple): $280 \mathrm{~nm}$

Run Notes:
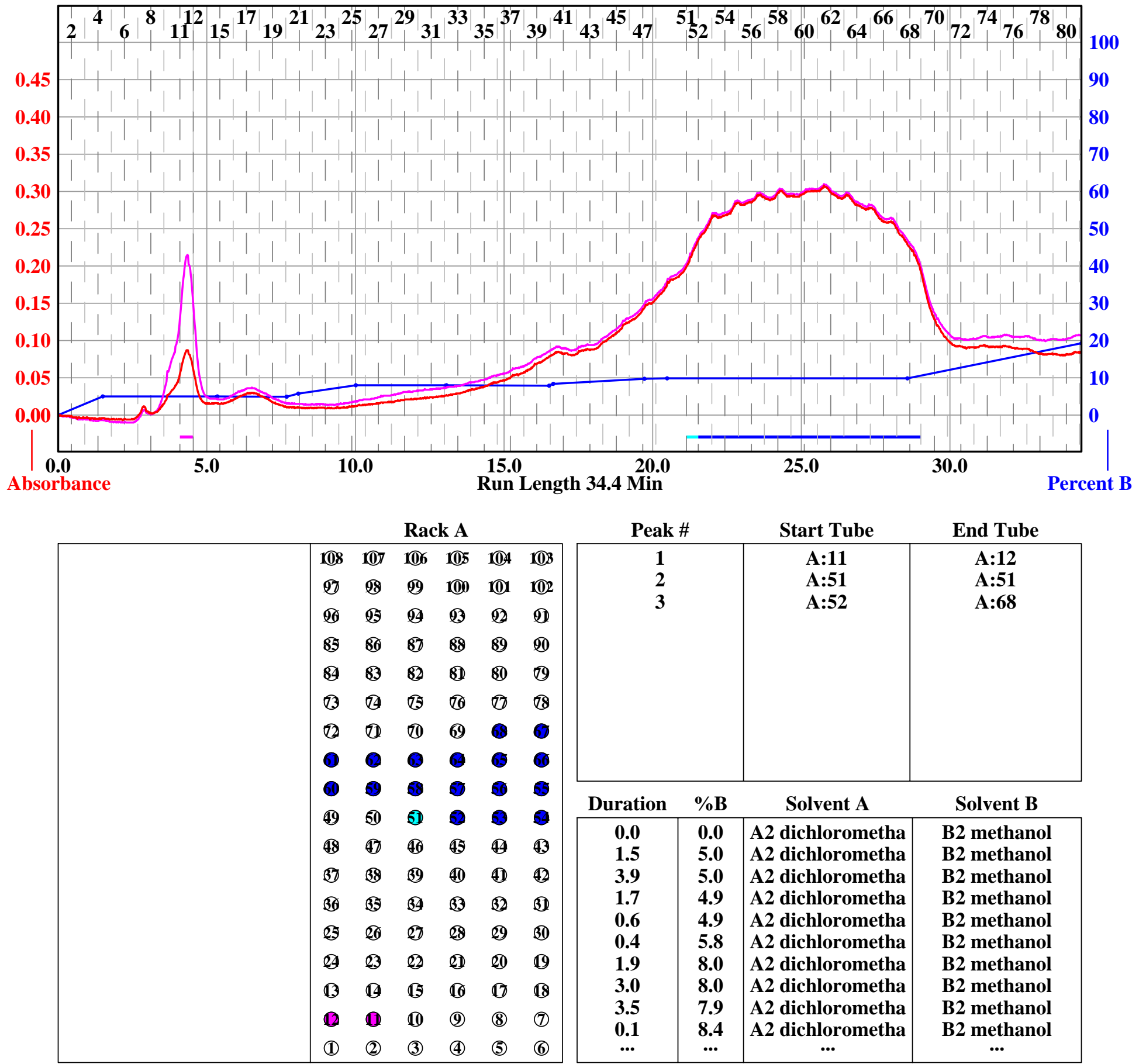

\begin{tabular}{|c|c|c|c|}
\hline \multicolumn{2}{|c|}{ Peak \# } & Start Tube & End Tube \\
\hline $\begin{array}{l}1 \\
2 \\
3\end{array}$ & & $\begin{array}{l}A: 11 \\
A: 51 \\
A: 52\end{array}$ & $\begin{array}{l}A: 12 \\
A: 51 \\
A: 68\end{array}$ \\
\hline Duration & $\% \mathbf{B}$ & Solvent A & Solvent B \\
\hline 0.0 & 0.0 & A2 dichlorometha & B2 methanol \\
\hline 1.5 & 5.0 & A2 dichlorometha & B2 methanol \\
\hline 3.9 & 5.0 & A2 dichlorometha & B2 methanol \\
\hline 1.7 & 4.9 & A2 dichlorometha & B2 methanol \\
\hline 0.6 & 4.9 & A2 dichlorometha & B2 methanol \\
\hline 0.4 & 5.8 & A2 dichlorometha & B2 methanol \\
\hline 1.9 & 8.0 & A2 dichlorometha & B2 methanol \\
\hline 3.0 & 8.0 & A2 dichlorometha & B2 methanol \\
\hline 3.5 & 7.9 & A2 dichlorometha & B2 methanol \\
\hline 0.1 & 8.4 & A2 dichlorometha & B2 methanol \\
\hline$\cdots$ & $\cdots$ & $\cdots$ & \\
\hline
\end{tabular}

$13 \mathrm{~mm} \times 100 \mathrm{~mm}$ Tubes 
Figure S72. 2-\{6-[5,6-Bis-(3-methoxy-phenyl)-[1,2,4]triazin-3-yl]-pyridin-2-yl\}-4-methyl-indole-1-carboxylic acid tertbatyplestez(20) 191

4x Column: 4

RediSep Column: Al2O3 pH=4 $8 \mathrm{~g}$

Flow Rate: $18 \mathrm{ml} / \mathrm{min}$

Equilibration Volume: $33.6 \mathrm{ml}$

Initial Waste: $0.0 \mathrm{ml}$

Air Purge: 1.0 min

Solvent: A2 hexanes

Solvent: B2 (3:1) ethyl

acetate:acetone
Peak Tube Volume: $8 \mathrm{ml}$

Non-Peak Tube Volume: $8 \mathrm{ml}$

Loading Type: Solid

Wavelength 1 (red): 254nm

Peak Width: 30 sec

Threshold: $0.20 \mathrm{AU}$

Wavelength 2 (purple): 280nm

Run Notes:
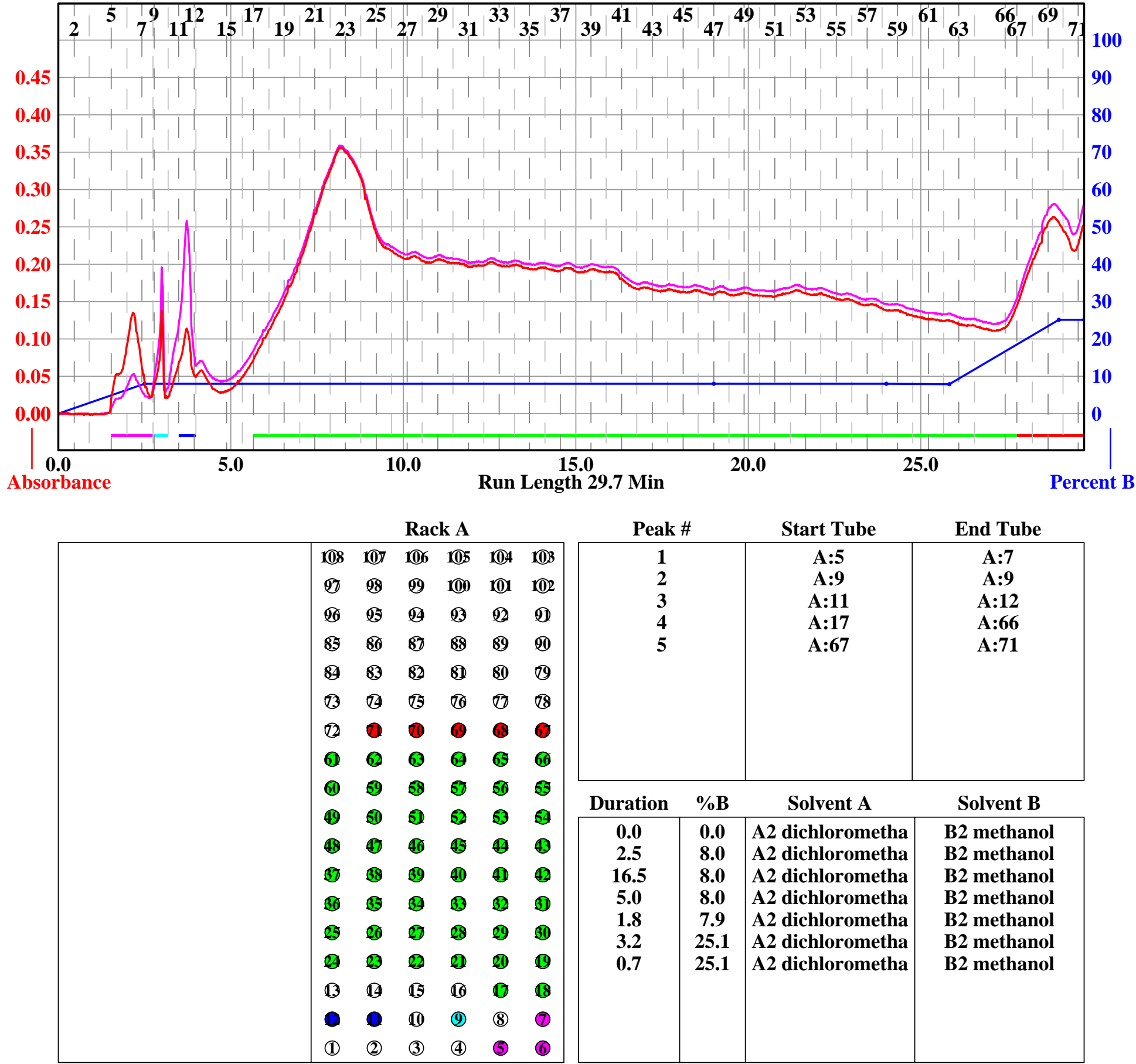

$13 \mathrm{~mm}$ x $100 \mathrm{~mm}$ Tubes

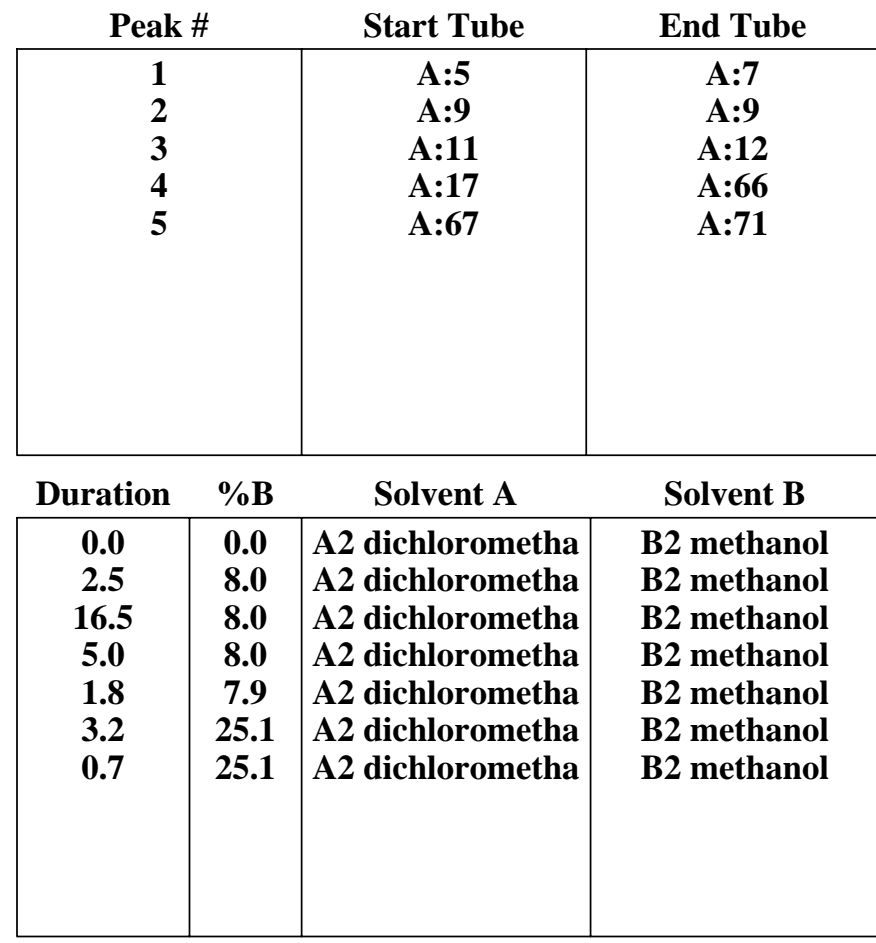


Figure S73. 2-\{6-[5,6-Bis-(4-butyl-phenyl)-[1,2,4]triazin-3-yl]-pyridin-2-yl\}-5-fluoro-indole-1-carboxylic acid tert-butyl Sorteply27ZG-B-155

Rf 200

Tuesday 26 February 2019 10:43AM

4x Column: 1

RediSep Column: Al2O3 pH=4 8g

Flow Rate: $18 \mathrm{ml} / \mathrm{min}$

Equilibration Volume: $33.6 \mathrm{ml}$

Initial Waste: $0.0 \mathrm{ml}$

Air Purge: 1.5 min

Solvent: A2 hexanes

Solvent: B2 (3:1) ethyl

acetate:acetone
Peak Tube Volume: $8 \mathrm{ml}$

Non-Peak Tube Volume: $8 \mathrm{ml}$

Loading Type: Solid

Wavelength 1 (red): 254nm

Peak Width: $30 \mathrm{sec}$

Threshold: 0.20 AU

Wavelength 2 (purple): 280nm

Run Notes:

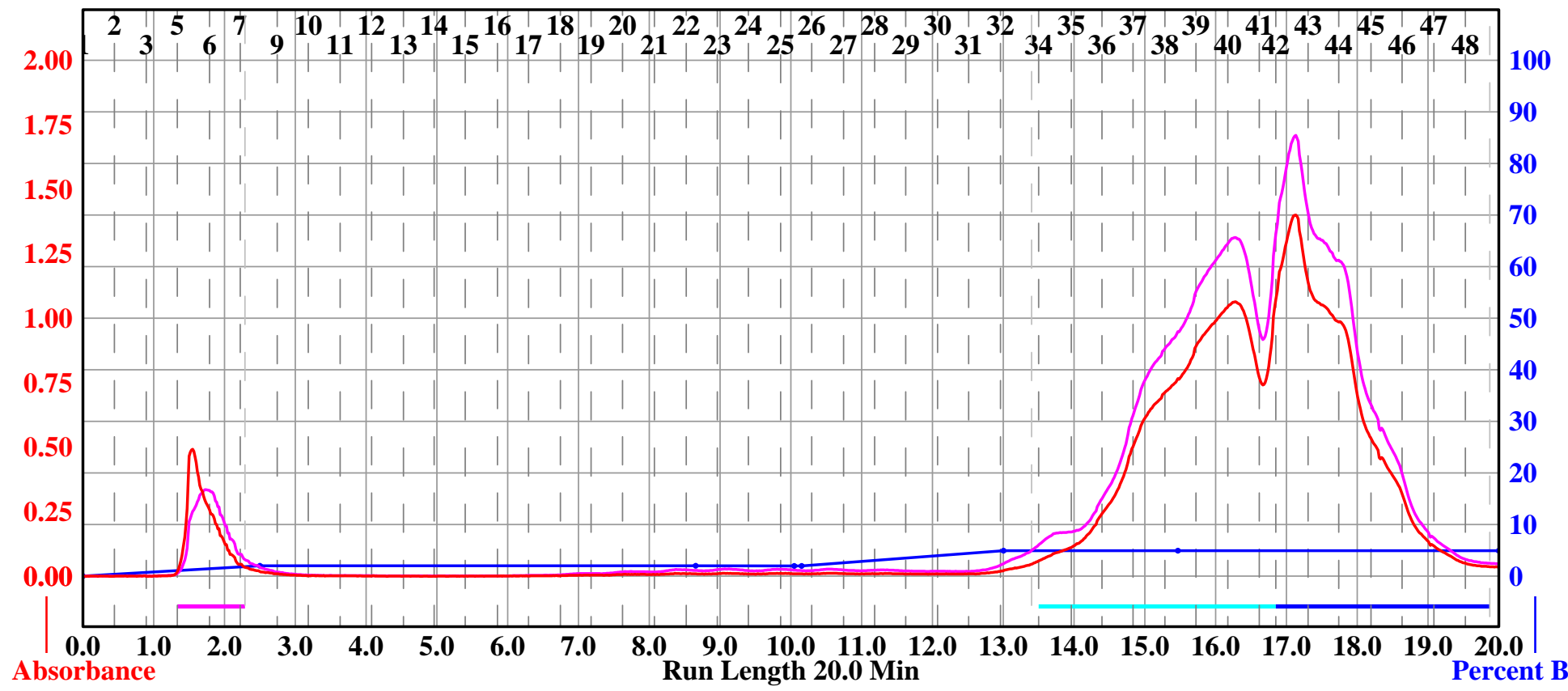

Rack A

Peak \#

Start Tube

End Tube

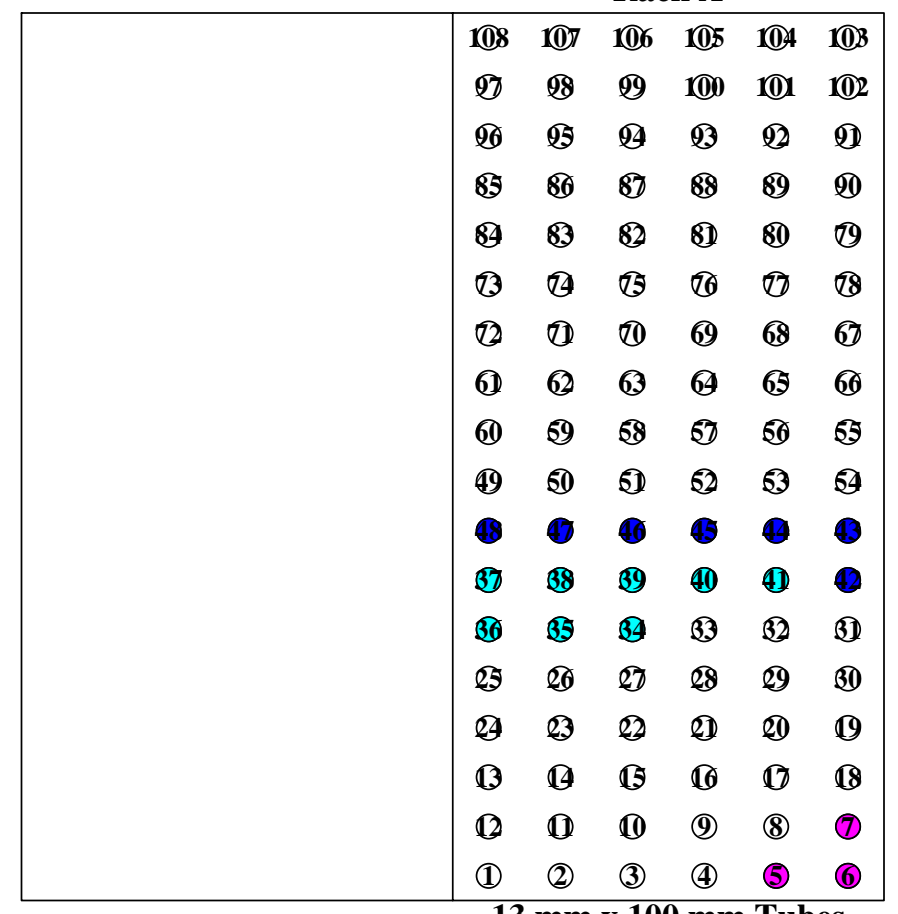

$13 \mathrm{~mm} \times 100 \mathrm{~mm}$ Tubes

\begin{tabular}{|c|c|c|c|}
\hline $\begin{array}{l}1 \\
\mathbf{2} \\
\mathbf{3}\end{array}$ & & $\begin{array}{c}\text { A:5 } \\
\text { A:34 } \\
\text { A:42 }\end{array}$ & $\begin{array}{c}\text { A:7 } \\
\text { A:41 } \\
\text { A:48 }\end{array}$ \\
\hline Duration & $\% \mathrm{~B}$ & Solvent A & Solvent B \\
\hline $\begin{array}{l}0.0 \\
2.5 \\
6.2 \\
1.4 \\
0.1 \\
2.9 \\
2.5 \\
4.5\end{array}$ & $\begin{array}{l}\text { 0.0 } \\
2.0 \\
2.0 \\
2.0 \\
2.0 \\
4.9 \\
4.9 \\
4.9\end{array}$ & $\begin{array}{l}\text { A2 dichlorometha } \\
\text { A2 dichlorometha } \\
\text { A2 dichlorometha } \\
\text { A2 dichlorometha } \\
\text { A2 dichlorometha } \\
\text { A2 dichlorometha } \\
\text { A2 dichlorometha } \\
\text { A2 dichlorometha }\end{array}$ & $\begin{array}{l}\text { B2 methanol } \\
\text { B2 methanol } \\
\text { B2 methanol } \\
\text { B2 methanol } \\
\text { B2 methanol } \\
\text { B2 methanol } \\
\text { B2 methanol } \\
\text { B2 methanol }\end{array}$ \\
\hline
\end{tabular}


Figure S74. 2-\{6-[5,6-Bis-(4-butyl-phenyl)-[1,2,4]triazin-3-yl]-pyridin-2-yl\}-5-methoxy-indole-1-carbox-ylic acid tert-butyl Samplei237G-B-157

4x Column: 1

RediSep Column: Al2O3 pH=4 8g

Flow Rate: $18 \mathrm{ml} / \mathrm{min}$

Equilibration Volume: $33.6 \mathrm{ml}$

Initial Waste: $0.0 \mathrm{ml}$

Air Purge: $1.5 \mathrm{~min}$

Solvent: A2 hexanes

Solvent: B2 (3:1) ethyl

acetate:acetone
Peak Tube Volume: $8 \mathrm{ml}$

Non-Peak Tube Volume: $8 \mathrm{ml}$

Loading Type: Solid

Wavelength 1 (red): 254nm

Peak Width: $30 \mathrm{sec}$

Threshold: 0.20 AU

Wavelength 2 (purple): $280 \mathrm{~nm}$

Run Notes:

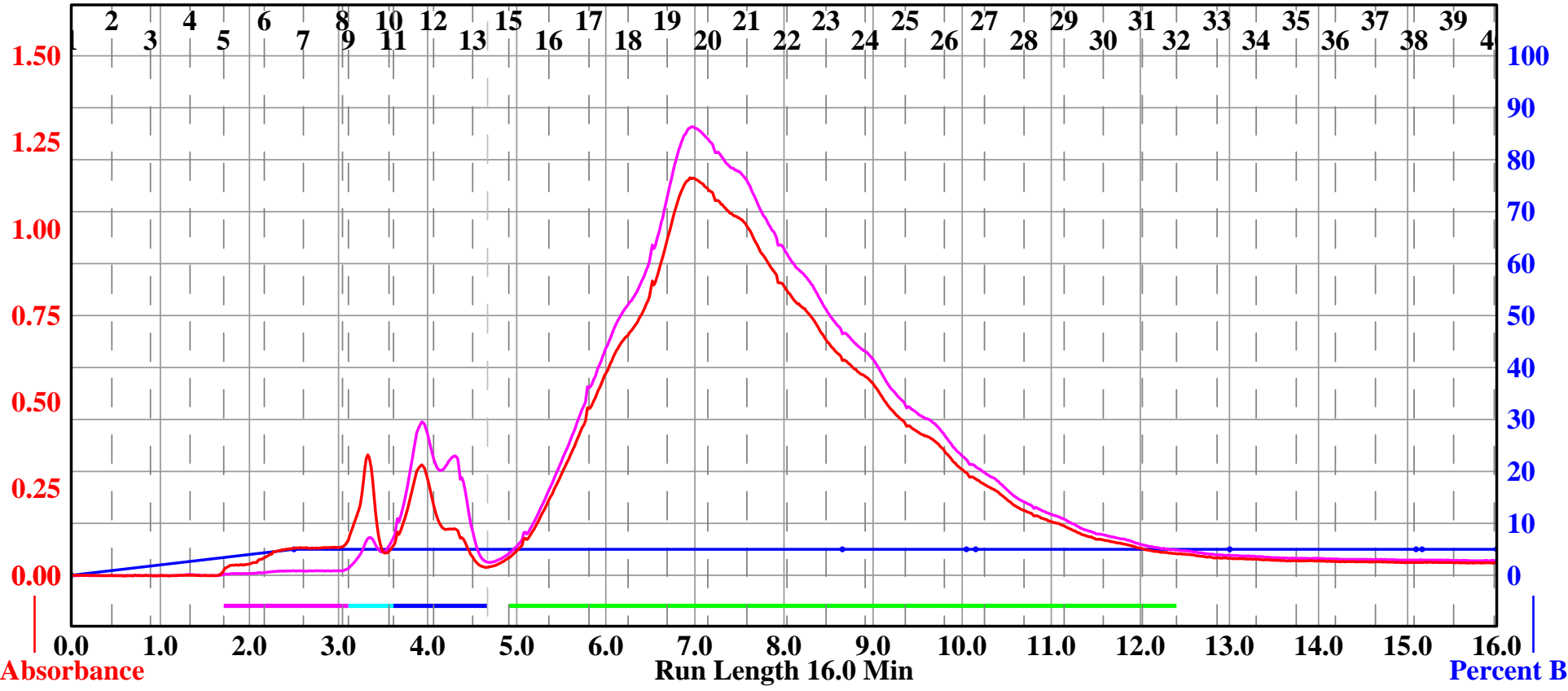

Peak \#

Start Tube

End Tube

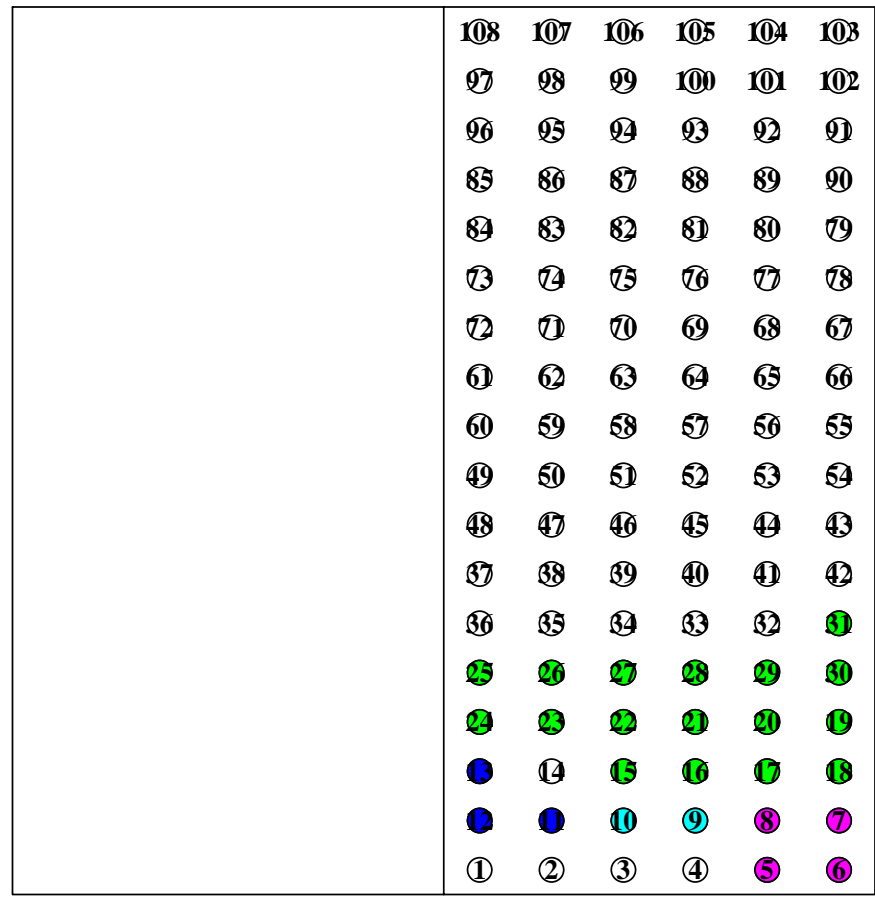

$13 \mathrm{~mm} \times 100 \mathrm{~mm}$ Tubes

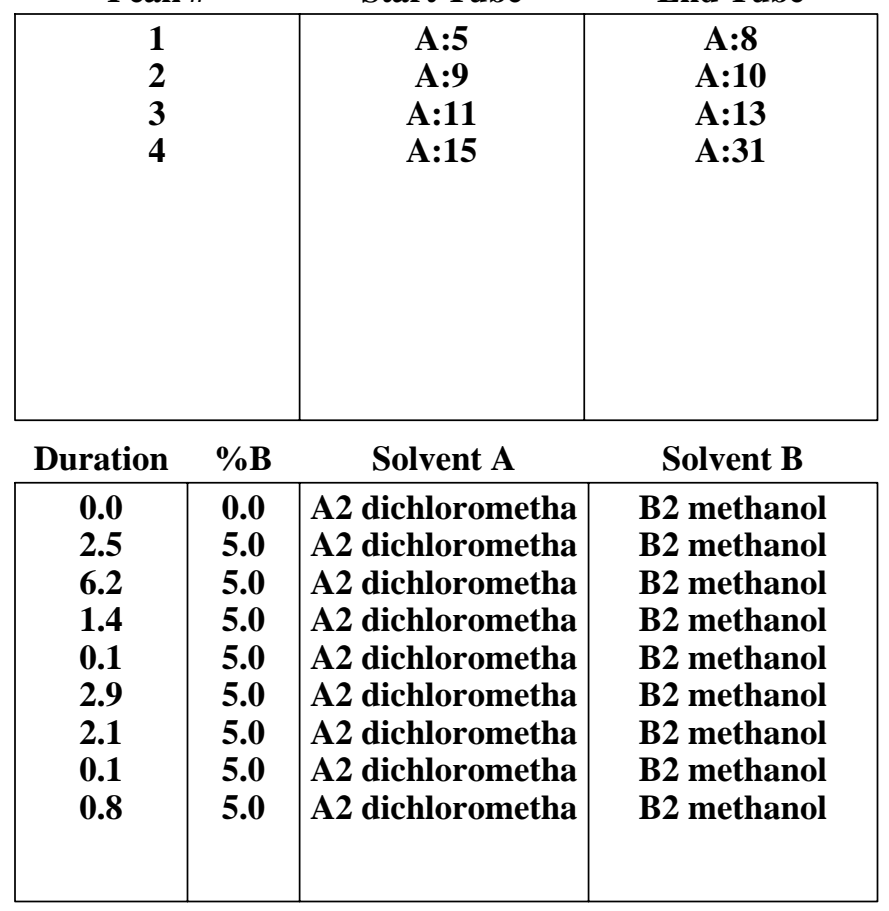


Figure S75. 6-Benzyloxy-2-(6-\{5,6-bis-[4-(3-methyl-butyl)-phenyl]-[1,2,4]triazin-3-yl\}-pyridin-2-yl)-indole-1-carboxylic acid tert-butyl ester (23)

Sample: ZZG-B-203

Rf 200

4x Column: 3

RediSep Column: Al2O3 pH=4 8g

Flow Rate: $18 \mathrm{ml} / \mathrm{min}$

Equilibration Volume: $33.6 \mathrm{ml}$

Initial Waste: $0.0 \mathrm{ml}$

Air Purge: 1.0 min

Solvent: A2 hexanes

Solvent: B2 (3:1) ethyl

acetate:acetone
Peak Tube Volume: $8 \mathrm{ml}$

Non-Peak Tube Volume: $8 \mathrm{ml}$

Loading Type: Solid

Wavelength 1 (red): 254nm

Peak Width: 30 sec

Threshold: 0.20 AU

Wavelength 2 (purple): 280nm

Run Notes:

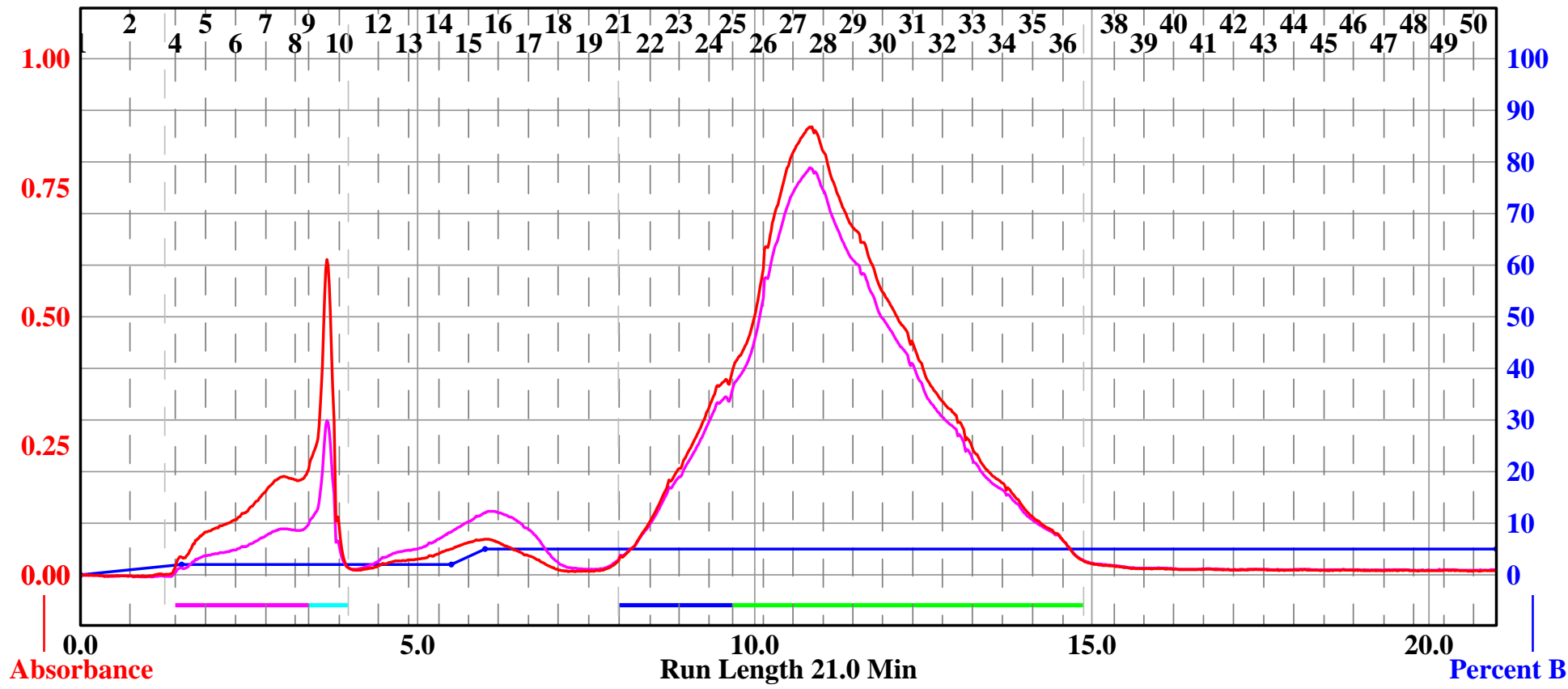

Rack A

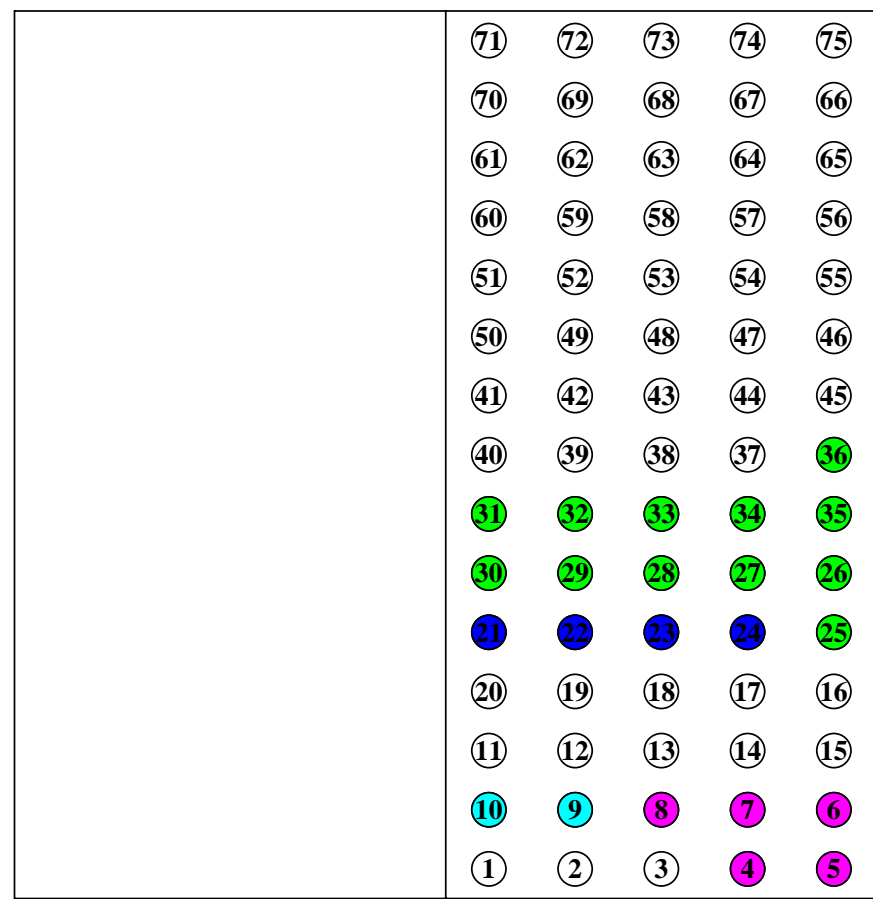

Friday 15 March 2019 01:51PM

\begin{tabular}{c|c} 
Peak \# & Start Tube \\
\hline 1 & A:4
\end{tabular}

End Tube

A:9

\begin{tabular}{l|l}
3 & A:21 \\
4 & A:25
\end{tabular}

\begin{tabular}{l|l}
3 & A:21 \\
4 & A:25
\end{tabular}

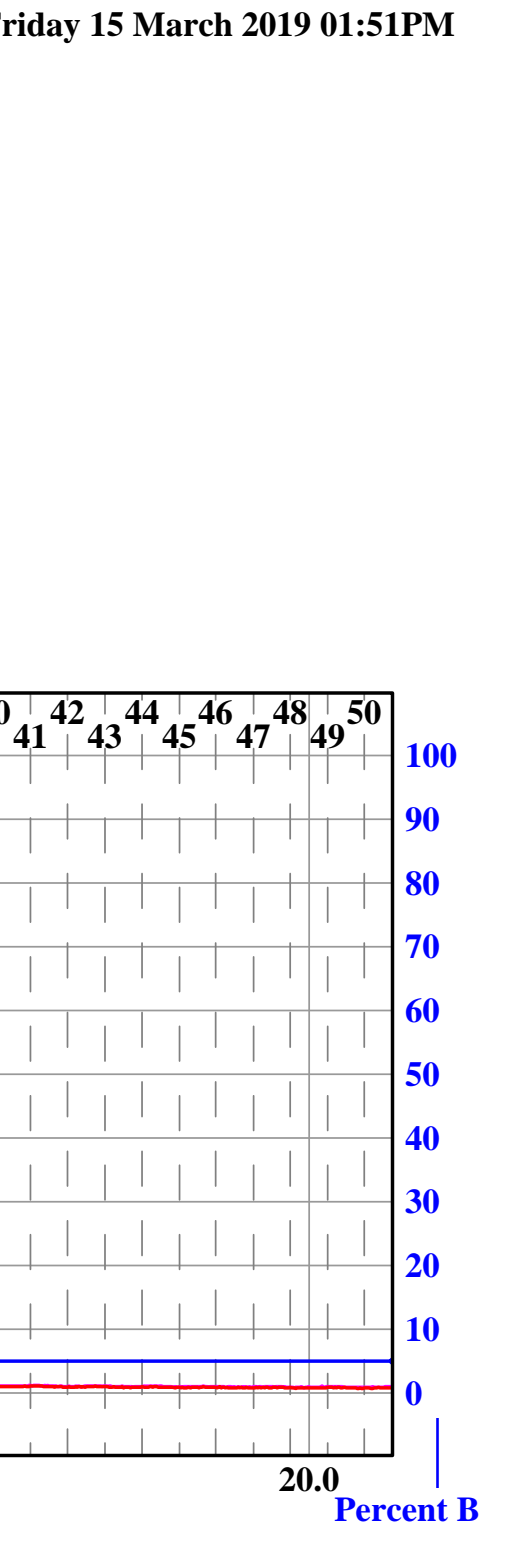

\begin{tabular}{|c|c|c|}
\hline 1 & A:4 & A:8 \\
2 & A:9 & A:10 \\
3 & A:21 & A:24 \\
4 & A:25 & A:36 \\
& & \\
& & \\
& & \\
\hline
\end{tabular}

$\begin{array}{llll}\text { Duration } & \% \text { B } & \text { Solvent A } & \text { Solvent B }\end{array}$

\begin{tabular}{|c|c|c|c|}
\hline $\mathbf{0 . 0}$ & $\mathbf{0 . 0}$ & A2 dichlorometha & B2 methanol \\
$\mathbf{1 . 5}$ & $\mathbf{2 . 0}$ & A2 dichlorometha & B2 methanol \\
$\mathbf{4 . 0}$ & $\mathbf{2 . 0}$ & A2 dichlorometha & B2 methanol \\
$\mathbf{0 . 5}$ & $\mathbf{5 . 0}$ & A2 dichlorometha & B2 methanol \\
$\mathbf{1 5 . 0}$ & $\mathbf{5 . 0}$ & A2 dichlorometha & B2 methanol \\
& & & \\
& & & \\
& & & \\
& & & \\
\hline
\end{tabular}


Figure S76. 2-(6-\{5,6-Bis-[4-(3-methyl-butyl)-phenyl]-[1,2,4]triazin-3-yl\}-pyridin-2-yl)-1H-indole (24)

Sample: ZZG-B-265

Rf 200

Friday 12 April 2019 05:04PM

4x Column: 4

RediSep Column: Al2O3 pH=4 8g

Flow Rate: $18 \mathrm{ml} / \mathrm{min}$

Equilibration Volume: $33.6 \mathrm{ml}$

Initial Waste: $0.0 \mathrm{ml}$

Air Purge: 0.5 min

Solvent: A1 hexane

Solvent: B2 (3:1) ethyl

acetate:acetone
Peak Tube Volume: Max.

Non-Peak Tube Volume: Max.

Loading Type: Solid

Wavelength 1 (red): 254nm

Peak Width: $30 \mathrm{sec}$

Threshold: 0.20 AU

Wavelength 2 (purple): $280 \mathrm{~nm}$

Run Notes:

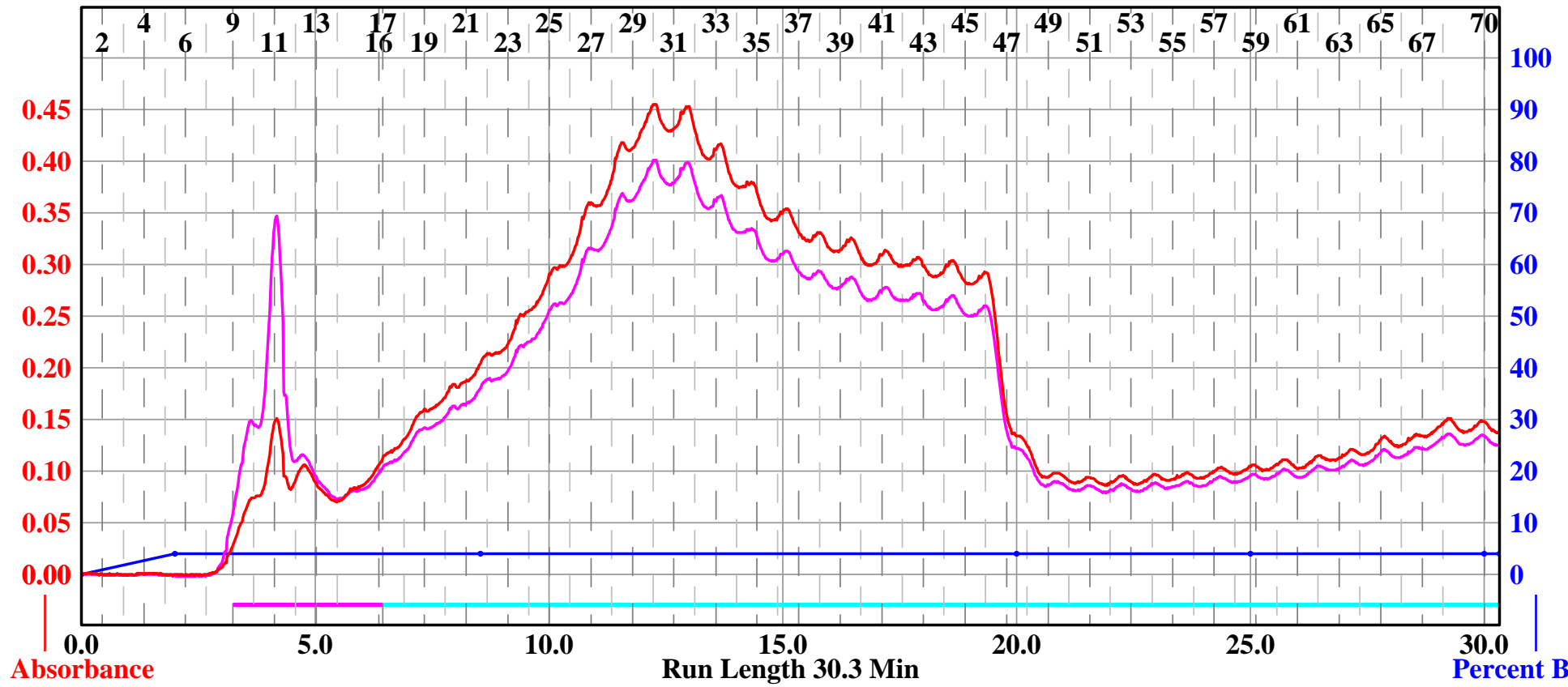

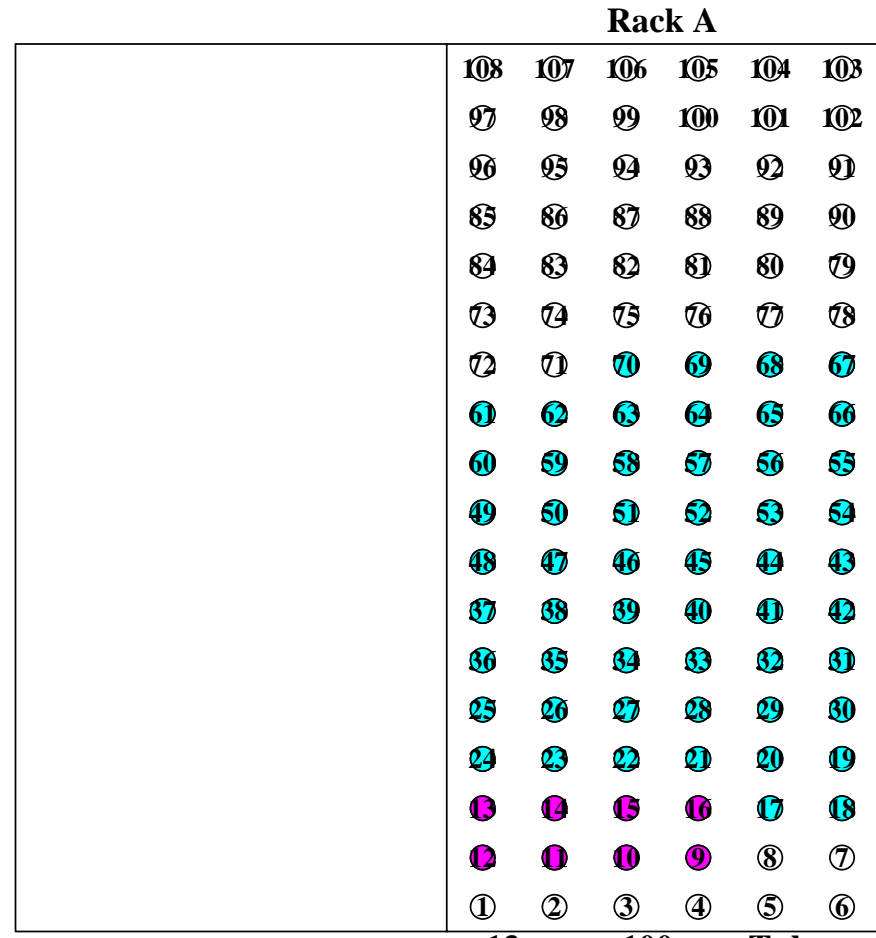

Start Tube

End Tube

\begin{tabular}{|c|c|c|c|}
\hline \multicolumn{2}{|c|}{ Peak \# } & Start Tube & End Tube \\
\hline \multicolumn{2}{|c|}{$\begin{array}{l}1 \\
2\end{array}$} & $\begin{array}{c}\text { A:9 } \\
\text { A:17 }\end{array}$ & $\begin{array}{l}\text { A:16 } \\
\text { A:70 }\end{array}$ \\
\hline Duration & $\% \mathrm{~B}$ & Solvent A & Solvent B \\
\hline 0.0 & 0.0 & A1 hexane & B2 methanol \\
\hline 2.0 & 4.0 & A1 hexane & B2 methanol \\
\hline 6.5 & 4.0 & A1 hexane & B2 methanol \\
\hline 11.5 & 4.0 & A1 hexane & B2 methanol \\
\hline 5.0 & 4.0 & A1 hexane & B2 methanol \\
\hline 5.0 & 4.0 & A1 hexane & B2 methanol \\
\hline 0.3 & 4.0 & A1 hexane & B2 methanol \\
\hline & & & \\
\hline & & & \\
\hline
\end{tabular}


Figure S77. 6-Benzyloxy-2-(6-\{5,6-bis-[4-(3,3-dimethyl-butyl)-phenyl]-[1,2,4]triazin-3-yl\}-pyridin-2-yl)-1H-indole (25) Sample: ZZG-B-175-3

4x Column: 3

RediSep Column: Al2O3 pH=4 8g

Flow Rate: $18 \mathrm{ml} / \mathrm{min}$

Equilibration Volume: $33.6 \mathrm{ml}$

Initial Waste: $0.0 \mathrm{ml}$

Air Purge: $0.5 \mathrm{~min}$

Solvent: A2 hexanes

Solvent: B2 (3:1) ethyl

acetate:acetone
Peak Tube Volume: Max.

Non-Peak Tube Volume: Max.

Loading Type: Solid

Wavelength 1 (red): 254nm

Peak Width: 30 sec

Threshold: 0.20 AU

Wavelength 2 (purple): 280nm

Run Notes:

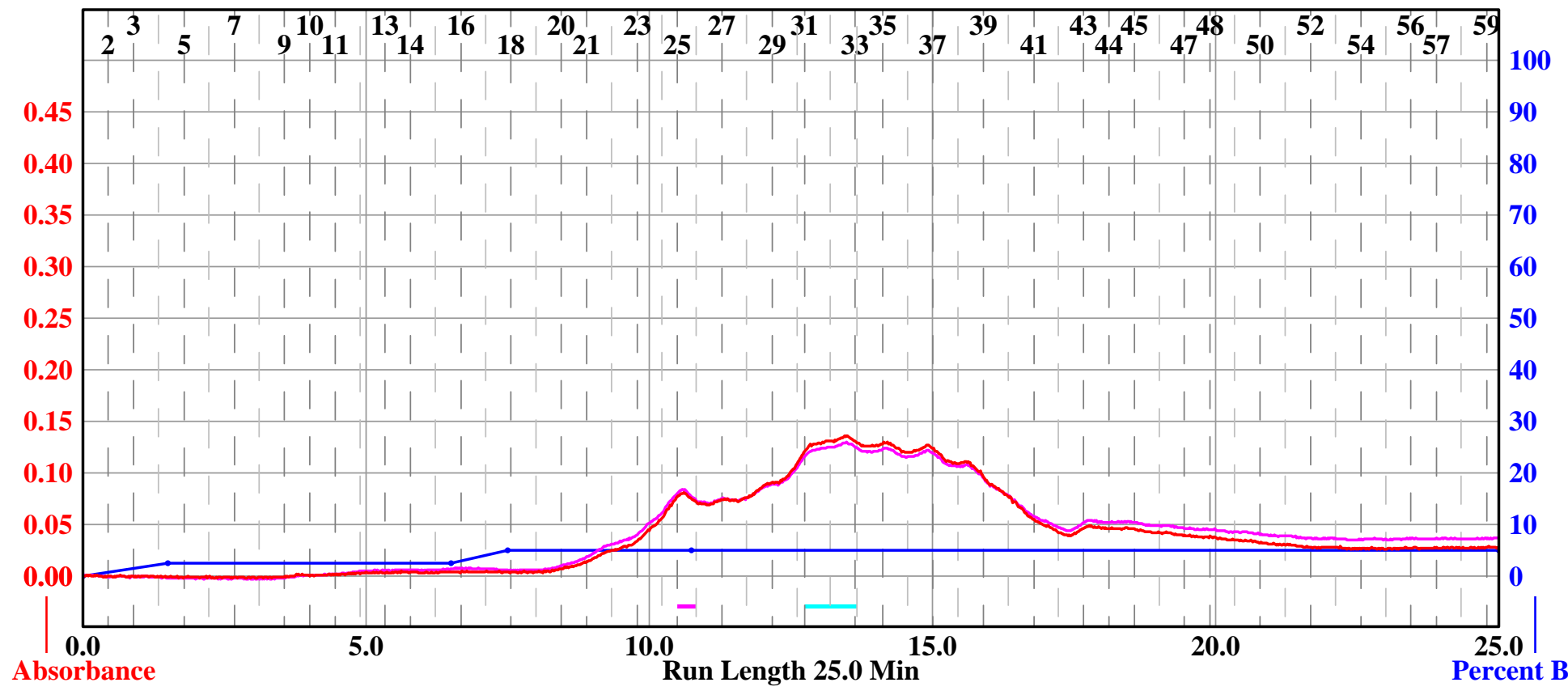

Rack A

Peak \#

Start Tube

End Tube

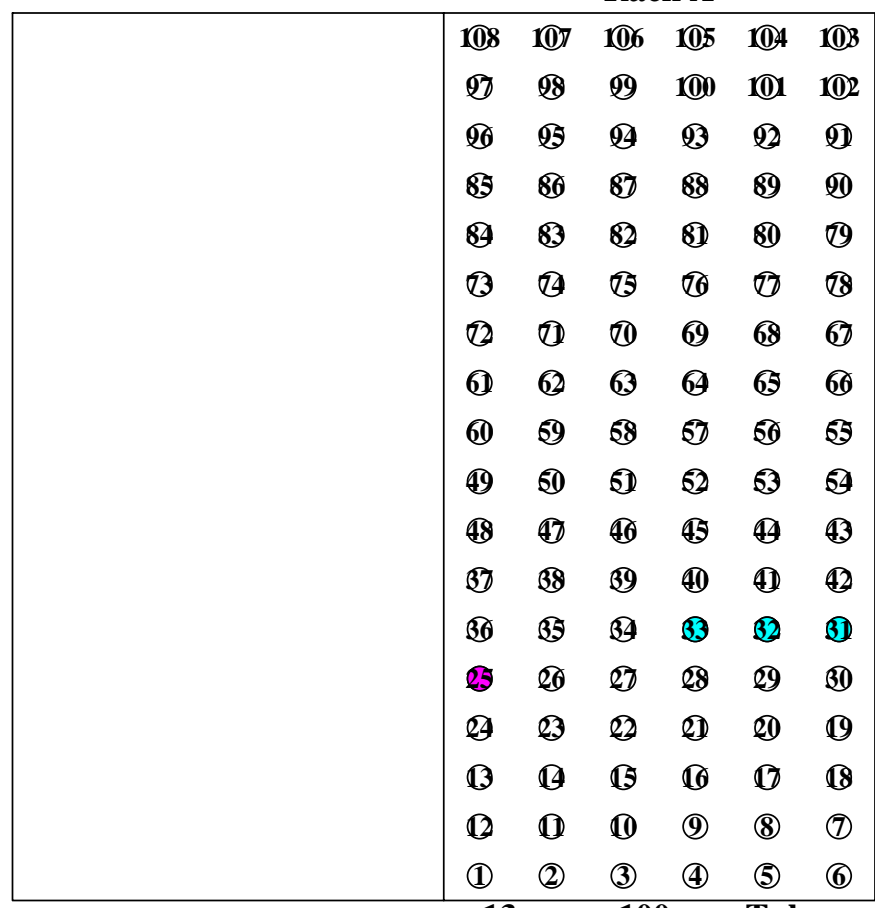

$13 \mathrm{~mm} \times 100 \mathrm{~mm}$ Tubes

\begin{tabular}{|c|c|c|c|}
\hline $\begin{array}{l}1 \\
2\end{array}$ & & $\begin{array}{l}\text { A:25 } \\
\text { A:31 }\end{array}$ & $\begin{array}{l}\text { A:25 } \\
\text { A:33 }\end{array}$ \\
\hline Duration & $\%$ B & Solvent A & Solvent B \\
\hline $\begin{array}{c}\mathbf{0 . 0} \\
1.5 \\
5.0 \\
1.0 \\
3.2 \\
14.3\end{array}$ & $\begin{array}{l}0.0 \\
2.5 \\
2.5 \\
5.0 \\
5.0 \\
5.0\end{array}$ & $\begin{array}{l}\text { A2 dichlorometha } \\
\text { A2 dichlorometha } \\
\text { A2 dichlorometha } \\
\text { A2 dichlorometha } \\
\text { A2 dichlorometha } \\
\text { A2 dichlorometha }\end{array}$ & $\begin{array}{l}\text { B2 methanol } \\
\text { B2 methanol } \\
\text { B2 methanol } \\
\text { B2 methanol } \\
\text { B2 methanol } \\
\text { B2 methanol }\end{array}$ \\
\hline
\end{tabular}


Figure S78. 5,6-Diphenyl-3-(6-phenyl-pyridin-2-yl)-[1,2,4]triazine (26)

Sample: ZZG-B-211

Rf 200

Wednesday 20 March 2019 04:04PM

4x Column: 4

RediSep Column: Al2O3 pH=4 8g

Flow Rate: $18 \mathrm{ml} / \mathrm{min}$

Equilibration Volume: $33.6 \mathrm{ml}$

Initial Waste: $0.0 \mathrm{ml}$

Air Purge: $1.0 \mathrm{~min}$

Solvent: A1 hexane

Solvent: B1 (3:1) ethyl

acetate:acetone
Peak Tube Volume: Max.

Non-Peak Tube Volume: Max.

Loading Type: Solid

Wavelength 1 (red): 254nm

Peak Width: $30 \mathrm{sec}$

Threshold: 0.20 AU

Wavelength 2 (purple): 280nm

Run Notes:

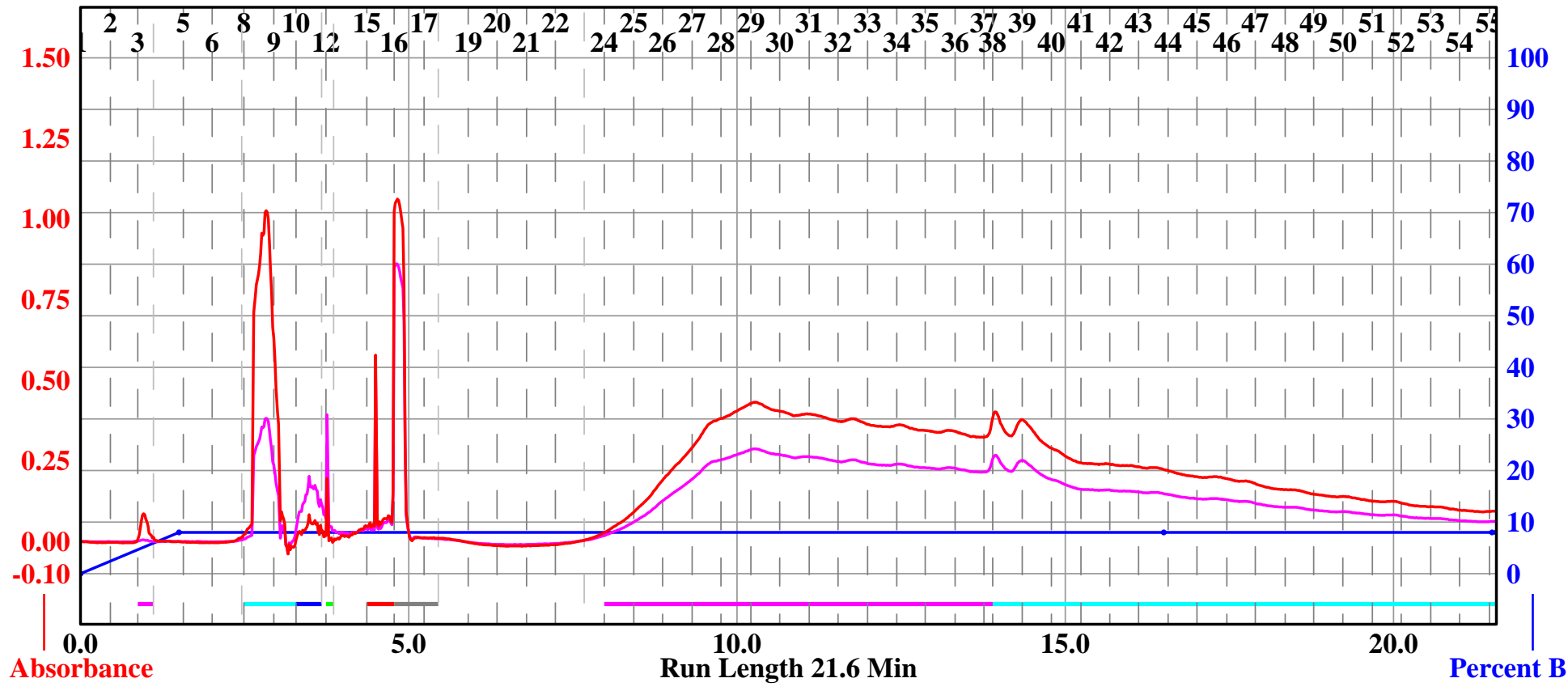

\begin{tabular}{|c|c|c|c|c|c|}
\hline \multicolumn{6}{|c|}{ Rack A } \\
\hline 108 & (107) & 106 & (105 & (10) & \\
\hline 20 & $\mathscr{Q}$ & 99 & (100 & (1) & \\
\hline df & (2) & (9) & (3) & (2) & \\
\hline 35 & 8 & 87 & 88 & & \\
\hline 89 & 8 & 82 & $\$$ & 80 & \\
\hline$(3)$ & (17) & 83 & (ש6) & (7) & \\
\hline 22 & (11) & (11) & (6) & 68 & \\
\hline (1) & (2) & (3) & (4) & 6 & \\
\hline 60 & 59 & 88 & 57 & ஏூ & \\
\hline (49) & (10) & ๒ & 52 & 3 & \\
\hline 48 & (47) & (40) & 49 & (4) & \\
\hline (37) & B8 & (39) & (41) & (41) & \\
\hline (36) & (35) & (3) & 3 & (32) & \\
\hline 25 & (2) & 8 & 8 & 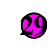 & \\
\hline (4) & 3 & (2) & (2) & (21) & \\
\hline (13) & (1) & (c) & (6) & (1) & \\
\hline (12) & (1) & 0 & (D) & (8) & \\
\hline (1) & (2) & (3) & (4) & (5) & \\
\hline
\end{tabular}

$13 \mathrm{~mm} \times 100 \mathrm{~mm}$ Tubes

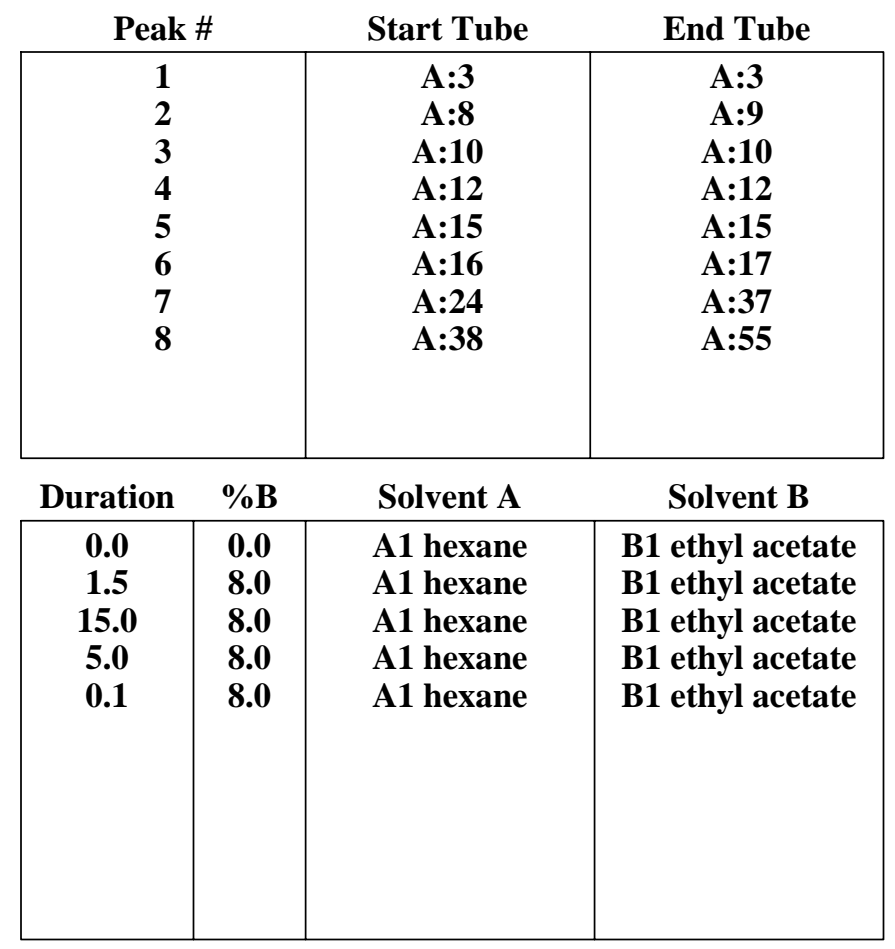

